\title{
Effect of Concurrent Walking and Interlocutor Distance on Conversational Speech Intensity and Rate in Parkinson's Disease
}

\author{
Cassandra M. McCaig, The University of Western Ontario \\ Supervisor: Dr. Scott Adams, The University of Western Ontario \\ A thesis submitted in partial fulfillment of the requirements for the Master of Science degree in \\ Health and Rehabilitation Sciences \\ (C) Cassandra M. McCaig 2013
}

Follow this and additional works at: https://ir.lib.uwo.ca/etd

Part of the Speech and Hearing Science Commons

\section{Recommended Citation \\ McCaig, Cassandra M., "Effect of Concurrent Walking and Interlocutor Distance on Conversational Speech Intensity and Rate in Parkinson's Disease" (2013). Electronic Thesis and Dissertation Repository. 1353. https://ir.lib.uwo.ca/etd/1353}

This Dissertation/Thesis is brought to you for free and open access by Scholarship@Western. It has been accepted for inclusion in Electronic Thesis and Dissertation Repository by an authorized administrator of Scholarship@Western. For more information, please contact wlswadmin@uwo.ca. 


\section{EFFECT OF CONCURRENT WALKING AND INTERLOCUTOR DISTANCE ON CONVERSATIONAL SPEECH INTENSITY AND RATE IN PARKINSON'S DISEASE}

(Thesis format: Monograph)

by

Cassandra Marie McCaig

Graduate Program in Health and Rehabilitation Sciences

A thesis submitted in partial fulfillment of the requirements for the degree of Master of Science

The School of Graduate and Postdoctoral Studies

Western University Canada

London, Ontario, Canada

(C) Cassandra M. McCaig (2013) 


\begin{abstract}
This study examined the effects of concurrent walking tasks and interlocutor distance on conversational speech production in fifteen individuals with idiopathic Parkinson's disease (PD) and fourteen age-equivalent controls. Recent studies of speech in PD have demonstrated that changes in the behavioural conditions and the environmental context can have a powerful effect on the severity of speech symptoms in PD. This investigation focused on changes in speech intensity and speech rate in response to changes in walking speed and interlocutor distance. Results suggest that the introduction of a concurrent walking task significantly increased the conversational speech intensity of both controls and individuals with PD. When compared to sitting and talking or standing and talking, current walking and talking appeared to have an energizing effect on conversational speech intensity. In addition, walking faster was associated with a significant increase in conversational speech intensity relative to normal and slow walking speeds. These results provide important new information about the effect of concurrent walking on speech motor performance and speech symptom severity in PD. The potential energizing effect of concurrent walking conditions on conversational speech intensity may be an important consideration in the assessment and treatment of individuals with low speech intensity in PD.
\end{abstract}

Keywords: Parkinson's disease, speech intensity, speech rate, concurrent tasks, interlocutor distance, stride length, walking speed 


\section{Acknowledgments}

First and foremost, I would like to express my sincere gratitude to my supervisor Dr. Scott Adams for introducing me to this field of study, for his encouragement and guidance, and for all his support along the way. I would also like to thank the other members of my committee, Dr. Allyson Dykstra and Dr. Mandar Jog, for their help and guidance, and for sharing their knowledge and precious time.

I would also like to thank my fellow speech and language science Master's/PhD candidates for their friendship and good conversation. It is easy to get lost and overwhelmed in the process, so thank you to Katie Findlater, Dayna Jablecki, Rebecca Affoo and Monika Andreetta for sharing in this journey and keeping me sane. Monika, without your uncanny organizational skills and attention to detail I would have missed many deadlines. Thank you for always keeping me informed - you were truly instrumental in the completion of this project.

A very special thank you goes out to my friends, particularly Jason, whose motivation and support, advice, and optimism helped keep me going, and for listening with 'genuine' interest when I described what I was working on.

I would like to thank my parents, Sam and Diane McCaig, and my grandfather Alex McCaig, who have always taught me that anything is possible, and for their endless love and support throughout my entire life. I owe them more than I could ever put into words.

Thank You. 


\section{Table of Contents}

Abstract ......................................................................................................................ii

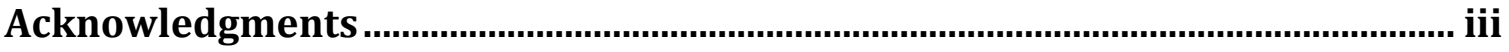

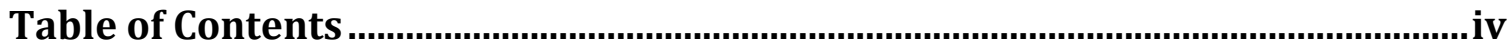

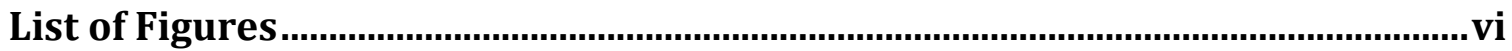

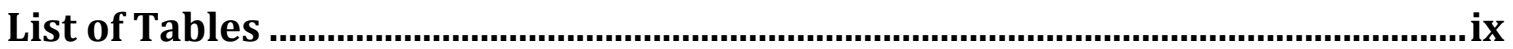

List of Appendices .........................................................................................................

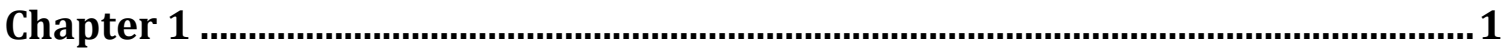

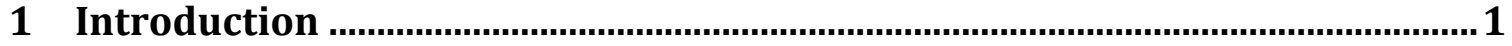

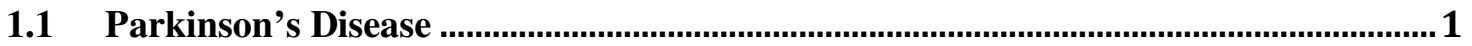

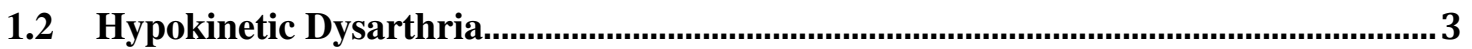

1.3 Speech Intensity associated with Hypokinetic Dysarthria .......................................... 4

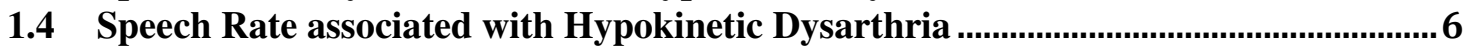

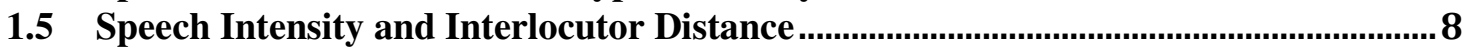

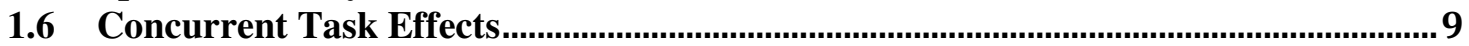

1.7 Concurrent Task Effects in Parkinson's Disease........................................................ 10

1.8 Speech and Concurrent Tasks in Parkinson's Disease................................................. 11

1.9 Walking Performance and Concurrent Task Effects in Parkinson's Disease .......... 14

1.10 Gait Hypokinesia in Parkinson's Disease: Velocity and Stride Length .................... 16

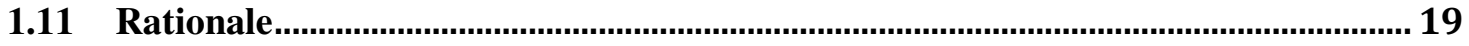

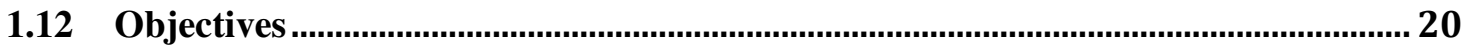

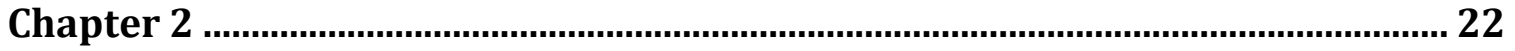

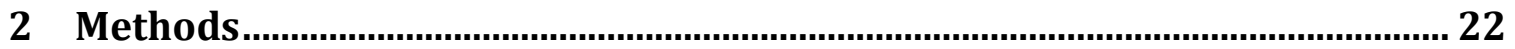

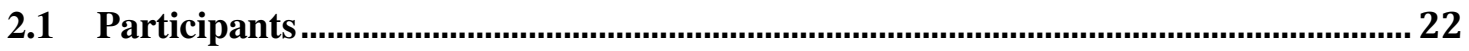

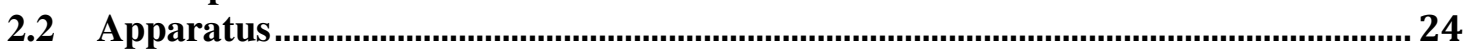

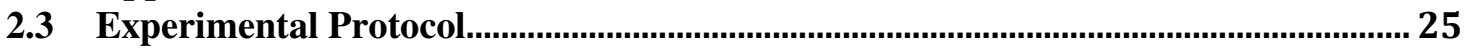

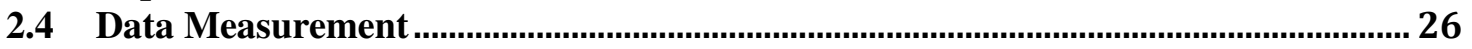

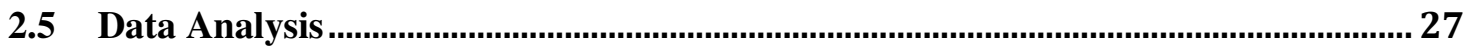

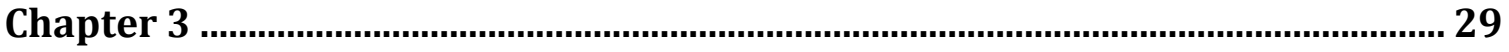

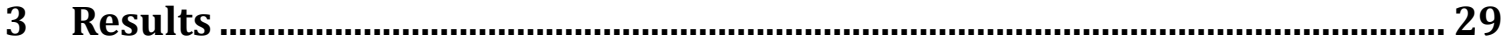

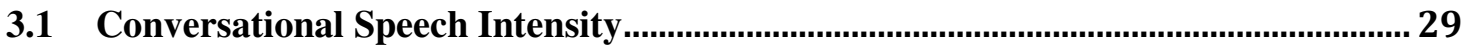

3.1.1 Main Effects: Group, Interlocutor Distance, and Walking Conditions.......................... 32

3.1.2 Interactions Related to Intensity: Group, Interlocutor Distance, and Walking

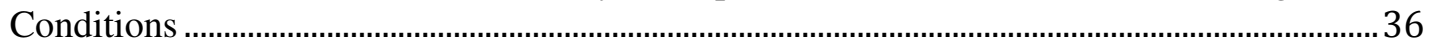

3.1.3 Post Hoc Comparisons: Walking Conditions, Walking Speeds ...................................... 39

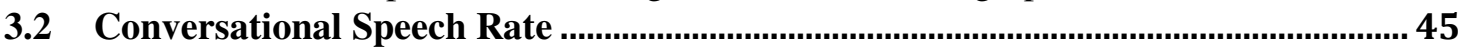

3.2.1 Main Effects Related to Speech Rate: Group, Interlocutor Distance, and Walking

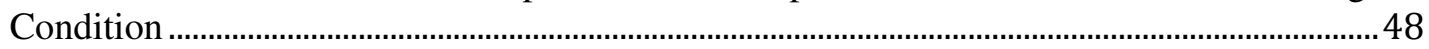


3.2.2 Interaction Related to Speech Rate: Group, Interlocutor Distance, and Walking Conditions

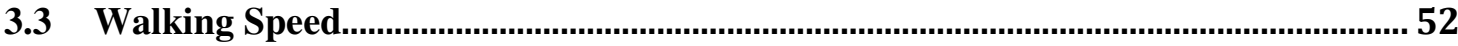

3.3.1 Main Effects Related to Walking Speed: Group, Talking Condition, and Walking Conditions

3.3.2 Interactions Related to Walking Speed: Group, Talking Condition, and Walking Conditions

3.3.3 Main Effects Related to Walking Speed: Interlocutor Distance

3.3.4 Interactions Related to Walking Speed: Group, Interlocutor Distance, and Walking

Condition

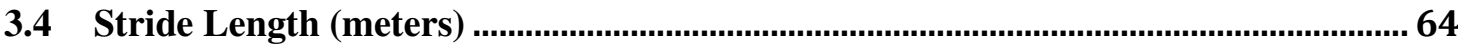

3.4.1 Main Effects Related to Stride Length: Group, Talking Condition, and Walking Condition .

3.4.2 Interactions Related to Stride Length: Group, Talking Condition, and Walking Condition .70

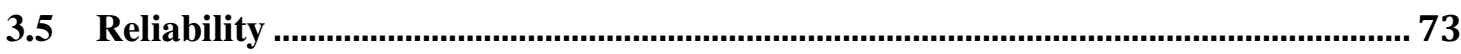

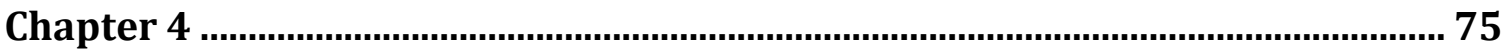

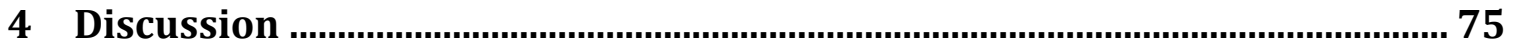

4.1 Effect of Concurrent Walking Tasks and Interlocutor Distance on Conversational

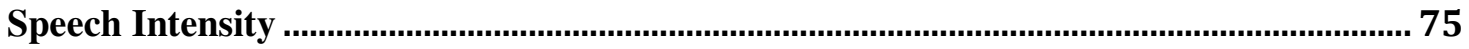

4.2 Effect of Concurrent Walking Tasks and Interlocutor Distance on Conversational

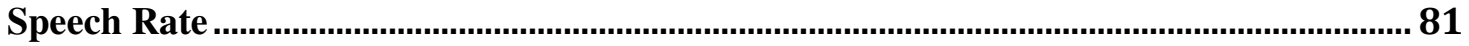

4.3 Effect of Concurrent Talking, Interlocutor Distance and Walking Condition on

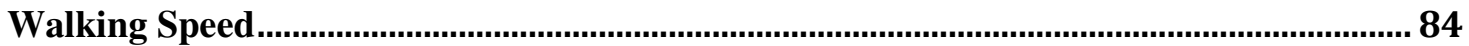

4.4 Effect of Concurrent Talking, Interlocutor Distance and Walking Condition on

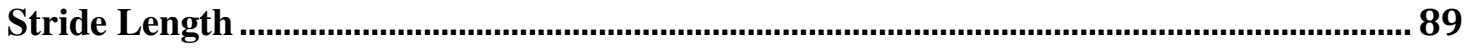

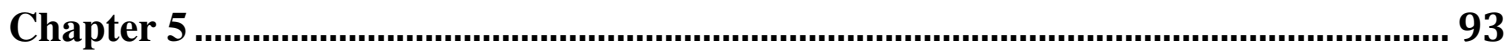

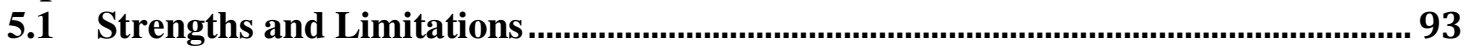

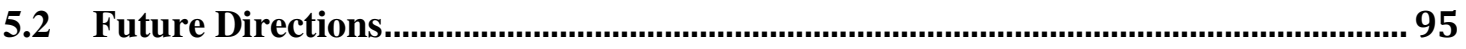

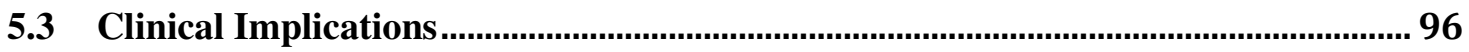

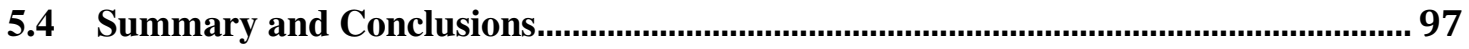

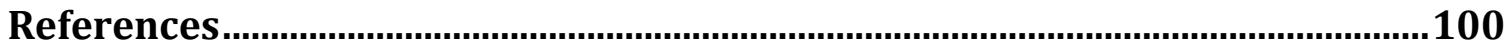

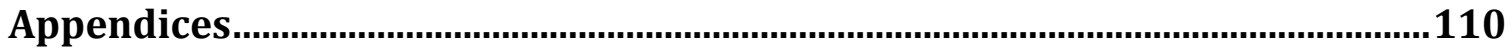

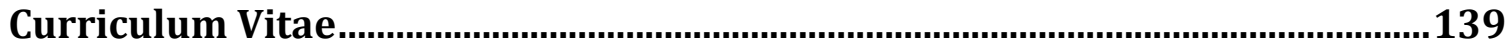




\section{List of Figures}

Figure 1. Average conversational speech intensity values by walking condition at an interlocutor distance of one meter. Standard deviations appear as error bars.

Figure 2. Average conversational speech intensity values by walking condition at an interlocutor distance of six meters. Standard deviations appear as error bars

Figure 3. Average conversational speech intensity values. Standard error scores appear as error bars.

Figure 4. Average conversational speech intensity of control and PD participants at an interlocutor distance of one and six meters. Standard error scores appear as error bars.

Figure 5. Average conversational speech intensity values across walking conditions.

Standard error scores appear as error bars.

Figure 6. Average conversational speech intensity across walking conditions at the one and six meter interlocutor distances for PD participants. Standard deviations appear as error bars

Figure 7. Average conversational speech intensity at one and six meters interlocutor distance across walking conditions for control participants. Standard deviations appear as error bars.

Figure 8. Average conversational speech intensity across walking speed conditions for control participants. Standard deviations appear as error bars.

Figure 9. Average conversational speech intensity across walking speed conditions for PD participants. Standard deviations appear as error bars.

Figure 10. Average conversational speech intensity in walking versus not-walking conditions for control participants. Standard deviations appear as error bars

Figure 11. Average conversational speech intensity in walking versus not-walking conditions for PD participants. Standard deviations appear as error bars.

Figure 12. Average conversational speech rate across walking conditions at an interlocutor distance of one meter. Standard deviations appear as error bars. 
Figure 13. Average conversational speech rate across walking conditions at an interlocutor distance of six meters. Standard deviations appear as error bars

Figure 14. Average conversational speech rate for control and PD participants. Standard error scores appear as error bars.

Figure 15. Average conversational speech rate at the one and six meter interlocutor distances. Standard error scores appear as error bars

Figure 16. Average conversational speech rate across walking conditions. Standard error scores appear as error bars.

Figure 17. Average walking speed by walking condition while concurrently talking.

Standard deviations appear as error bars.

Figure 18. Average walking speed by walking condition while not talking. Standard deviations appear as error bars.

Figure 19. Average walking speed for control and PD participants. Standard error scores appear as error bars

Figure 20. Average walking speed while concurrently talking and walking, and walking only. Standard error scores appear as error bars.

Figure 21. Average walking speed across walking conditions for control and PD

participants. Standard error scores appear as error bars.

Figure 22. Average walking speed by walking condition at an interlocutor distance of one meter. Standard deviations appear as error bars.

Figure 23. Average walking speed by walking condition at an interlocutor at an interlocutor distance of six meters. Standard deviations appear as error bars

Figure 24. Average walking speed at an interlocutor distance of one and six meters.

Standard error scores appear as error bars

Figure 25. Average stride length by walking condition while concurrently talking.

Standard deviations appear as error bars

Figure 26. Average stride length by walking condition while not talking. Standard deviations appear as error bars. 66

Figure 27. Average stride length for control and PD participants. Standard error scores appear as error bars 
Figure 28. Average stride length while concurrently talking and walking, and walking

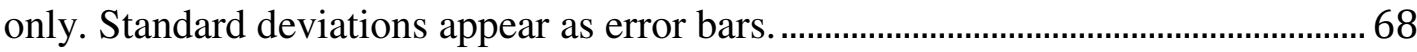

Figure 29. Average stride length across walking speed conditions. Standard deviations

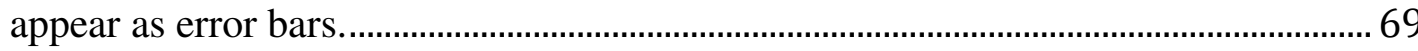

Figure 30. Average stride length by walking condition at an interlocutor distance of one meter. Standard deviations appear as error bars........................................................... 72

Figure 31. Average stride length by walking condition at an interlocutor distance of six meters. Standard deviations appear as error bars 


\section{List of Tables}

Table 1. PD Participant Demographic Information .............................................................. 23

Table 2. Control Participant Demographic Information....................................................... 23

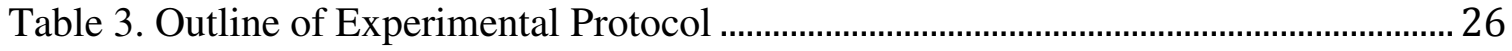

Table 4. Average Conversational Speech Intensity Values by Walking Condition at an

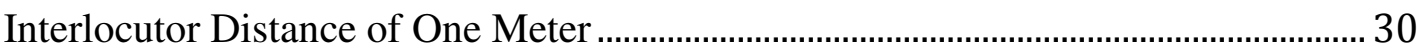

Table 5. Average Conversational Speech Intensity Values by Walking Condition at an Interlocutor Distance of Six Meters............................................................................. 31

Table 6. Average Conversational Speech Intensity Values.................................................... 33

Table 7. Average Conversational Speech Intensity of Control and PD Participants at an Interlocutor Distance of One and Six Meters...................................................................... 34

Table 8. Average Conversational Speech Intensity Values Across Walking Conditions. 35

Table 9. Average Conversational Speech Intensity Across Walking Conditions at the One and Six Meter Interlocutor Distances for PD Participants ............................................ 37

Table 10. Average Conversational Speech Intensity at One and Six Meters Interlocutor Distance Across Walking Conditions for Control Participants ....................................... 38

Table 11. Average Conversational Speech Intensity Across Walking Speed Conditions

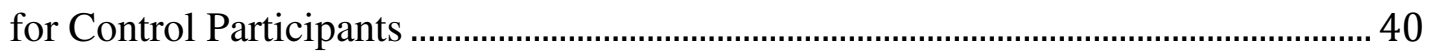

Table 12. Average Conversational Speech Intensity Across Walking Speed Conditions

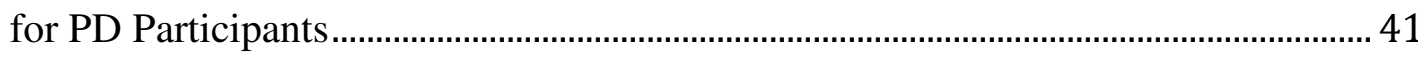

Table 13. Average Conversational Speech Intensity in Walking versus Not-Walking Conditions for Control Participants.

Table 14. Average Conversational Speech Intensity in Walking Versus Not-Walking Conditions for PD Participants.

Table 15. Average Conversational Speech Rate Across Walking Conditions at an Interlocutor Distance of One Meter.

Table 16. Average Conversational Speech Rate Across Walking Conditions at an

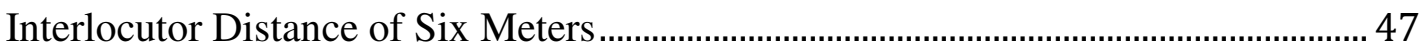

Table 17. Average Conversational Speech Rate for Control and PD participants 49 
Table 18. Average Conversational Speech Rate at the One and Six Meter Interlocutor

Distances.

Table 19. Average Conversational Speech Rate Across Walking Conditions..................... 51

Table 20. Average Walking Speed by Walking Condition while Concurrently Talking 53

Table 21. Average Walking Speed by Walking Condition while not Talking .................... 54

Table 22. Average Walking Speed for Control and PD Participants................................... 56

Table 23. Average Walking Speed while Concurrently Talking and Walking, and

Walking Only 57

Table 24. Average Walking Speed Across Walking Speed Conditions for Control and PD Participants 58

Table 25. Average Walking Speed by Walking Condition at an Interlocutor Distance of

One Meter. 60

Table 26. Average Walking Speed by Walking Condition at an Interlocutor Distance of Six Meters. 61

Table 27. Average Walking Speed at an Interlocutor Distance of One and Six Meters... 63

Table 28. Average Stride Length by Walking Condition while Concurrently Talking .... 65

Table 29. Average Stride Length by Walking Condition while not Talking 66

Table 31. Average Stride Length While Concurrently Talking and Walking, and Walking Only 68

Table 32. Average Stride Length Across Walking Speed Conditions 69

Table 33. Average Stride Length by Walking Condition at an Interlocutor Distance of

One Meter.

Table 34. Average Stride Length by Walking Condition at an Interlocutor Distance of Six Meters 72

Table 35. Inter and Intra-Judge Reliability 74 


\section{List of Appendices}

Appendix A. Western University Ethics Approval.................................................................110

Appendix B. PD Participant Letter of Information................................................................111

Appendix C. Control Participant Letter of Information ........................................................114

Appendix D. Participant Consent Form ……………………………………………….....117

Appendix E. Participant Intake Survey ………………………………………………...118

Appendix F. 3 Way ANOVA: Speech Intensity.................................................................119

Appendix G. T-Test: Effect of Walking Speed on Speech Intensity..................................122

Appendix H. 3 Way ANOVA: Speech Rate.......................................................................124

Appendix I. 3 Way ANOVA: Walking Speed and Concurrent Talking...............................127

Appendix J. 3 Way ANOVA: Walking Speed and Interlocutor Distance ..........................130

Appendix K. 3 Way ANOVA: Stride Length and Concurrent Talking...............................133

Appendix L. 3 Way ANOVA: Stride Length and Interlocutor Distance ............................136 


\section{Chapter 1}

\section{$1 \quad$ Introduction}

\subsection{Parkinson's Disease}

Parkinson's disease (PD) is a highly variable, chronic progressive neurological disorder characterized by a number of movement related symptoms. The four cardinal motor features of PD include resting tremor, muscle rigidity, bradykinesia or akinesia, and postural and gait instability (Darley, Aronson, \& Brown, 1975). Additional motor features include hypomimia (masked face), dysphagia, dysarthria, shuffling gait, motor freezing, festination of gait movements, and reduced arm-swing during walking (Darley et al., 1975). There are also many non-motoric symptoms of PD, these include anxiety, fatigue, sleep disturbance, pain/numbness in limbs, as well as behavioral and mental symptoms such as depression, decreased motivation, slowed thinking, and a decline in cognition that can progress to dementia (Fahn, 2003).

Symptoms of PD result from the progressive degeneration of dopaminergic neurons in the substantia nigra (SN) located within the basal ganglia (BG) (Fahn, 2003). The $\mathrm{SN}$ is responsible for producing dopamine and transmitting it into the striatum (also located within the BG), where it is then released as a neurotransmitter that is responsible for smooth coordinated movement. Post-mortem analyses clearly show shrinkage and loss of the pigmented cells of the $\mathrm{SN}$ and degeneration in additional brain regions (Braak, 2003). Symptoms do not typically appear until dopamine concentration has decreased by $80 \%$, which corresponds to a loss of approximately $50 \%-60 \%$ of dopaminergic neurons 
(Hamani \& Lozano, 2003; Bernheimer, Birkmayer, Hornykiewicz, Jellinger, \& Seitelberger, 1973).

A definitive diagnosis can only be confirmed post-mortem because there is no biomarker that can identify the disease in a living person. Currently PD diagnosis is based on the presence of at least two of the four cardinal motor features and a good response to levodopa medication (Cooperman, Forwell, \& Hugos, 2002; Dirette, 2000; Lim, Van Wegen, de Goede, Jones, Rochester, Hetherington, Nieuwboer, Willems, \& Kwakkel, 2005). Melvin Yahr and Margaret Hoehn were the first to develop a system for grading severity of PD (Hoehn \& Yahr, 1967). There are a number of scales to describe disease progression and response to treatment in PD, such as the Modified Hoehn and Yahr Scale, Unified Parkinson's Disease Rating Scale (UPDRS), Schwab \& England Activities of Daily Living scale, and the Parkinson's disease Questionnaire (PDQ-39). PD is a complex disorder, and the exact cause of neural degradation is still unknown. Studies suggest that multiple etiologies occur in PD, involving interactions among age, gender, environmental, and genetic factors (Korell \& Tanner, 2005). Strong evidence suggests that the risk of PD increases with age, and that prevalence is consistently higher in men than women (1.5:1) (Korell \& Tanner, 2005; Tanner \& Goldman, 1996; Wirdefeldt, Adami, Cole, Trichopoulos, \& Mandel, 2011). A number of methods have been used to assess the genetic contribution of PD, such as twin studies, familial studies, and genetic association studies (Wirdefeldt et al., 2011). Twin studies suggest low concordance rates, while familial studies indicate that family history is a strong risk factor for PD (Wirdefeldt et al., 2011). Genetic association studies have shown fairly consistent evidence that some genes are more important for susceptibility of 
PD (Wirdefeldt et al., 2011). A number of studies also suggest increased risk of PD to particular occupational and environmental factors (see Wirdefeldt et al. (2011) for review). Wirdefeldt et al. (2011) examined particular exposures of interest and categorized their epidemiological evidence (Wirdefeldt et al., 2011). According to this analysis, evidence is limited on the role of metals, organic solvents, magnetic fields, and increased body mass index on increasing the risk of PD, while more suggestive evidence was found for pesticides and the intake of dairy products (Wirdefeldt et al., 2011). Coffee and smoking were both found to reduce the risk of PD (Wirdefeldt et al., 2011).

PD typically develops in older adults (50 to 70 years old, with the mean age of 60) although it has affected individuals as young as 30 to 40 years old. According to Parkinson Society Canada (2010), approximately 100,000 Canadians currently live with the disease, and this is projected to double by 2016. The prevalence in the general population is estimated to be between 1 and 2 cases per 1,000 (Weiner \& Lang, 1989). For individuals aged 65 and older the prevalence rises to approximately 10 in 1,000 (Tanner \& Goldman, 1996).

\subsection{Hypokinetic Dysarthria}

It is estimated that $60-80 \%$ of individuals with PD will develop a speech impairment directly related to PD (Adams \& Dykstra, 2009). Hypokinetic dysarthria is a motor speech disorder that is most commonly associated with PD (Adams \& Dykstra, 2009), although it can manifest in other neurodegenerative disorders such as progressive supranuclear palsy (PSP), Shy-Drager syndrome, and multi-system atrophy (MSA). The term dysarthria is the collective name for a variety of speech disorders involving any or 
all of the basic speech processes, including respiration, phonation, resonance, articulation, and prosody (Blanchet, 2002). The term 'hypokinesia' describes the nature of the movement disorder and generally refers to reduced range and force of movement. Hypokinetic speech is characterized by reduced range of oral movements, slowed speed of single movements, increased speed of repetitive movements with limited range, and reduced force of movement of the speech articulators (Darley et al., 1975).

The most distinctive features of hypokinetic dysarthria were originally reported by Darley, Aronson and Brown (1969a), and include a reduction in pitch range and inflections (monopitch), reduced stress on stressed syllables, monoloudness, imprecise consonants, inappropriate silences, short rushes of speech, variable speech rate, and harsh/breathy voice quality. An additional feature that was not in the original description is low speech intensity (hypophonia). Overall, speech in PD is attenuated in range, and restricted in speed and flexibility (Duffy, 2005). These speech symptoms often impair effective communication, as they have a negative impact on speech intelligibility, social functioning and emotional well-being (Miller, Noble, \& Jones, 2006; Sapir, Spielman, Ramig, Story, \& Fox, 2007). These communication impairments ultimately affect the quality of life in individuals with PD (Schrage, Jahanshahi, \& Quinn, 2000).

\subsection{Speech Intensity associated with Hypokinetic Dysarthria}

It is estimated that $40-50 \%$ of individuals with hypokinetic dysarthria will present with low speech intensity (hypophonia), and it is often the first speech symptom to become apparent in the early stages of the disease (Adams, Winnell, \& Jog, 2010). Hypophonia is associated with a reduction in speech intensity of $2-5 \mathrm{~dB}$ relative to healthy 
older adults (Adams et al., 2010). Many studies have investigated the nature of hypophonia in the speech of individuals with $\mathrm{PD}$, and the various contexts and environments that exacerbate or minimize this effect. Ho, Iansek, and Bradshaw (1999b) found that individuals with hypophonia had a greater reduction in intensity when the speech output was more attentionally demanding (conversation) than when reciting wellpracticed sentences. Ho, Bradshaw, Iansek, and Alfredson (1999a), Ho, Bradshaw, and Iansek (2000), and Adams et al. (2010) investigated the ability of individuals with hypophonia to regulate speech intensity in background noise. These studies found that individuals with PD demonstrated relatively normal patterns of intensity regulation despite an overall reduction in intensity relative to controls. These studies also gave evidence that some individuals with PD have the same capacity for loud speech as healthy controls. In Adams et al.'s (2010) investigation, average speech intensity levels for control participants in quiet conditions were $70 \mathrm{~dB}$, while the PD participants had a speech intensity of $65 \mathrm{~dB}$ in the same condition. When the PD participants were placed in an environment with $65 \mathrm{~dB}$ of background noise, they were able to generate a speech intensity of $70 \mathrm{~dB}$. This provides evidence that individuals with PD can speak at intensity level appropriate for non-noisy conditions if cued properly.

The pathophysiological mechanism that causes hypophonia in PD is still unclear. Some evidence suggests that the reduction in vocal intensity is directly related to a reduction in respiratory effort and a reduction in the adductory force of the vocal folds, resulting in insufficient pressure build-up to generate loud speech (Duffy, 2005). As a result, the primary goal of many behavioral speech treatments is to increase speech intensity by consciously training individuals to increase their vocal fold adductory force 
and their respiratory effort. In addition, it has been suggested that hypophonia may be linked to a perceptual deficit or a sensorimotor integration deficit (Ho et al., 2000). For example, Ho et al. (2000) examined the perceived loudness of one's own speech using immediate and delayed playback procedures and found that individuals with PD overestimated the perceived loudness of their own speech. This suggests that perceptual deficits may play a role in the hypophonia associated with PD.

\subsection{Speech Rate associated with Hypokinetic Dysarthria}

A number of previous studies have investigated typical habitual speaking rates in healthy and disordered populations. Venkatagiri (1999) found the speech rate of reading in young healthy adults to be 188.1 words per minute (WPM) or 262 syllables per minute (SPM), and the rate of discourse to be 143.3 WPM or 195.5 SPM. Lutz and Mallard (1986) reported similar findings for the speech rate of reading (198 WPM or 254 SPM) and discourse (158.6 WPM or 216.6 SPM). Similarly, Duchin and Mysak (1987) reported that the mean conversational speaking rate was 182.7 WPM or 236 SPM in healthy older adults.

Rapid speaking rate is a prominent and distinctive perceptual feature of hypokinetic dysarthria (Duffy, 2005). A number of speech features characteristic of hypokinetic dysarthria, such as reduced stress on stressed syllables, imprecise consonant articulation, short rushes and inappropriate silences, create articulatory distortions in the speech signal. As a result, dysarthric speech is frequently perceived to be faster than it actually is (Yorkston, Hammen, \& Beukelman, 1990). Many studies have investigated habitual speaking rates of individuals with hypokinetic dysarthria in PD, and results are 
inconsistent. For example, a number of studies suggest that individuals with PD exhibit greater than normal speaking rates (McRae \& Tjaden, 2002). However, these studies are primarily perceptual in nature, and attribute this perceived increase in speaking rate to a blurring of acoustic parameters in connected speech (McRae \& Tjaden, 2002). In addition, there have been previous reports that speech in PD may be associated with a reduction in the size of the acoustic working space, which degrades perceptual impressions (Weismer, Jeng, Laures, Kent, \& Kent, 2001; McRae \& Tjaden, 2002). As a result, methods of rate reduction in PD have been a common treatment to improve speech intelligibility, as slowed speaking rates are associated with an expansion of acoustic working space (Weismer et al., 2001).

In contrast, a number of studies have revealed that the speaking rate between individuals with PD and healthy adults is similar. For example, Walsh and Smith (2012) examined speaking rate in 16 individuals with PD and 16 control participants. Their findings demonstrate no significant difference between groups, however they reported that the PD participants had a larger range of speaking rate. Similarly, Flint, Black, Campbell-Taylor, Gailey and Levington (1992) examined speaking rate (syllables per second) of connected speech in 30 individuals with PD and 31 normal controls. Their findings suggest that both the PD and control groups have similar speaking rates for long passages. However, when reading short sentences, participants displayed a slight increase in speaking rate although this finding did not reach statistical significance.

Based on this summary, it would appear that individuals with PD have a slightly faster speaking rate than healthy adults when reading shorter sentences, but as the length of connected speech increases (reading paragraphs and passages), speaking rate may 
become more similar. Unfortunately, there is little information related to speaking rate during conversational speech in individuals with PD.

\subsection{Speech Intensity and Interlocutor Distance}

The ability to alter speech intensity with changes in interlocutor distance is an important aspect of natural communication. According to Zahorik and Kelly (2007), speech intensity obeys an inverse square law with distance. That is, when distance between speakers is doubled, there is a corresponding $6 \mathrm{~dB}$ reduction in speech volume due to sound propagation losses. In healthy controls, Michael, Siegel and Pick (1995) suggested that speakers make prosodic, pragmatic and semantic changes in addition to increasing speech volume to accommodate changes in interlocutor distance. These compensatory changes are very similar to the 'Lombard' response, which explains that speech intensity is adjusted to compensate for increases in background noise. To facilitate effective communication, healthy controls adjust vocal output to compensate for the pragmatic demands of the speaking environment.

A few previous studies have examined the ability of individuals with PD to regulate speech intensity in response to manipulations in interlocutor distance. Ho et al. (1999a) examined intensity regulation in both healthy controls and participants with PD for two speech tasks, conversation and reciting sentences. Despite an overall reduction in intensity, individuals with PD demonstrated relatively normal patterns of intensity regulation in response to changes in interlocutor distance. Interestingly, the overall reduction in intensity was more pronounced in the conversational speech task than when reciting sentences. The authors suggested that during conversation, less attention is 
available for speech production. Similarly, Adams et al. (2010) found that healthy controls and individuals with PD produced similar changes in conversational speech intensity over changes in interlocutor distance. These previous studies suggest that changes in interlocutor distance may have a similar effect on speech intensity in PDs and controls and that the attentional demands of a speech task may influence this interlocutor distance effect. Unfortunately, no previous studies have attempted to systematically examine the potential interaction between changes in attention demands and changes in interlocutor distance. One potential method of investigating this interaction could involve the use of a dual-task paradigm (i.e., walking and talking) combined with variations in interlocutor distance.

\subsection{Concurrent Task Effects}

Despite the large body of research that has indicated consistent findings for concurrent task interference in normal participants, the factors that influence this interference are less well understood (Holmes, Jenkins, Johnson, Adams, \& Spaulding, 2010). Researchers in the field of cognitive psychology have examined concurrent task or divided attention phenomenon for several decades, and a number of theories have been developed to account for the task interference (or lack there-of) (Dromey \& Shim, 2008). The capacity theories suggest that concurrent task interference occurs because the two tasks compete for attentional resources ('attention' refers to the focus of mental activity on a task) (Wu \& Hallett, 2009), and thus one or both of the tasks will have a performance decrement. The time-sharing model suggests that attention is shifted back and forth between the two tasks in a series of smooth and rapid transitions, and the 
functional distance hypothesis states that there will be less interference when the two tasks activate neural networks that are farther apart from one another (Dromey \& Shim, 2008).

However, there have been reports of improved performance on one or both activities when performed concurrently. For example, the energizing hypothesis proposed by Adams et al. (2010) suggests that a concurrent motor task may improve the performance of a motor-speech task in individuals with PD. Recent studies on neurologically impaired participants have assessed the language-motor system interaction to determine whether performing an activity in one domain can facilitate the activity in another (Dromey \& Shim, 2008; Meinzer, Breitenstein, Westerhoff, Sommer, Rosser, Rodriguez, Harnish, \& Floel, 2011). Results suggest a link between linguistic functions and the activation of motor areas, indicating that these two systems share functional neural resources (Meinzer et al., 2011). For example, Meinzer et al. (2011) investigated the word retrieval performance of individuals with aphasia while sitting versus standing, and found improved performance while standing. The authors suggested that preactivation of the motor cortex could be used to excite the speech/language network. The enhancing or energizing effect of the language network has also been seen in healthy populations. For example, Dromey and Shim (2008) found that speech intensity increased in healthy controls when performed concurrently with a motor task.

\subsection{Concurrent Task Effects in Parkinson's Disease}

Performing two tasks simultaneously is a common and typically unconscious activity of daily living, for example, talking while walking. To successfully perform tasks 
concurrently, the body relies on several regions of the cerebral cortex and the basal ganglia. The cerebral cortex is believed to be responsible for controlling conscious motor activities, such as the acquisition of a new motor skill. As these motor activities become habitual, they are thought to be controlled unconsciously by the basal ganglia (Seitz \& Roland, 1992). Idiopathic PD results from basal ganglia dysfunction, and as a consequence, task automaticity is reduced and cognitive resources must be drawn on to maintain performance of both tasks. Studies investigating the impact of concurrent tasks on gait in PD reveal lack of automaticity and increased cognitive demands (Rochester, Nieuwboer, Baker, Hetherington, Willems, Kwakkel, Wegen, Lim, \& Jones, 2008). Consequently, individuals with basal ganglia dysfunction will have difficulty coping with concurrent tasks, and performance deficits are often exacerbated in individuals with PD (Rochester et al., 2008).

\subsection{Speech and Concurrent Tasks in Parkinson's Disease}

Speech tasks have been primarily included as a dual-task paradigm for cognitive loading in studies investigating gait in individuals with PD. As a result, the emphasis in data collection and analysis has been primarily focused on gait performance, and not on the speech performance. The few studies that have provided analysis for the speech task have been inconsistent, and the type of speech task may explain this variability. For example, Galletly and Brauer (2005) had participants with idiopathic PD walk for ten minutes at a comfortable pace while performing serial-3 subtraction and verbal fluency tasks. Both speech tasks improved significantly when performed concurrently with the walking task (16\% and $73 \%$ respectively), while there was a decrement in walking performance. A similar study by O'Shea, Morris, and Iansek (2002) found a decrement in 
both speech (serial-3 subtraction) and walking task. In addition, a study by Yogev, Giladi, Peretz, Sprinter, Simon, and Hausdorff (2005) had participants with PD walk for two minutes down a 25-meter corridor at a comfortable pace while performing separate verbal-cognitive tasks: listening to a tape and answering questions, and performing serial3 subtraction. Results demonstrated performance decrements in both the speech and walking task. In all studies, speech performance was quantified as the percentage of errors in the serial subtraction tasks. Unfortunately, these measures of speech performance reflect speech from a language perspective, and do not reflect a motor speech perspective or a speech acoustic perspective.

Only two studies have been conducted to determine the effects of a concurrent task that is specifically focused on motor speech production in PD, and the results are inconsistent. Ho, Iansek, and Bradshaw (2002) examined the effect of a concurrent manual task on speech intensity in individuals with PD using two speech tasks, conversation and a loud numerical recitation task. The concurrent task in this study was a visuomotor manual tracking task, which required participants to monitor the position of a randomly moving target with a joystick. Results indicated a significant decrease in speech intensity during the loud number recitation speech task but not during conversational speech. Adams et al. (2010) examined conversational speech intensity while participants performed a concurrent visuomotor manual tracking task in which participants tracked a moving target with a handheld bulb in various interlocutor distances and multi-talker background noise conditions. The PD participants produced a significant increase in conversational speech intensity while performing the concurrent motor task, while the healthy controls produced a significant reduction in speech 
intensity. The authors suggested that certain concurrent tasks might have an energizing effect on speech intensity in individuals with PD. In addition, the authors suggest that an increase in speech intensity seen during the concurrent manual task might have been the result of "an overall increase in effort" that was caused by the introduction of a concurrent task (Adams et al., 2010; Dromey \& Bates, 2005; Dromey \& Shim, 2008).

There have been a few previous reports that examined the relationship between concurrent motor tasks and speech intensity in healthy controls. In two studies of healthy young controls, Dromey and Bates (2005) and Dromey and Shim (2008) found that a concurrent manual task was associated with a significant increase in the speech intensity of spoken sentences at typical conversational intensity. Gentilucci, Benuzz, Gangitano, and Grimaldi (2001) investigated the effect of grasping an object on syllable production, and found that increases in the size of concurrent hand grasping movements were associated with increased speech intensity. In addition, Gentilucci (2003) examined 'observed' grasp movements on syllable production and found similar results. The authors suggest that the cortical areas responsible for observation and preparation of grasp movements are partially shared with the cortical areas involved in speech production.

Many concurrent task studies investigating motor speech performance have employed simple motor tasks involving the upper extremities (e.g., finger-tapping, motor tracking), but it is difficult to extrapolate from these to typical human behavior (Dromey \& Shim, 2008). For example, O'Shea et al. (2002) state that upper limb movements are primarily controlled by motor cortical regions and require attention, visual guidance and somatosensory feedback to control their performance. In contrast, locomotion consists of 
highly pre-programmed movements, and is thought to be regulated at the brainstem, spinal, and cerebellar regions (O'Shea et al., 2002).

\subsection{Walking Performance and Concurrent Task Effects in Parkinson's}

\section{Disease}

It is estimated that $80-90 \%$ of individuals with PD will develop gait impairments within the first three years of diagnosis (Kang, Brostein, Masterman, Redeings, Crum, \& Ritz, 2005). Decreased mobility and physical functioning have significant consequences on quality of life in PD, and are rated among the worst aspects of the disease (Schrag et al., 2000; Post, Merkus, J. de Haan, \& Speelman, 2007; Kelly, Eusterbrock, \& ShumwayCook, 2012).

Walking in the real world is a very complex activity that requires cognitive flexibility to meet the changing demands of the environment (Rochester et al., 2008). This creates additional challenges for individuals with basal ganglia dysfunction (i.e., PD), as substantial conscious control is needed for the walking performance, making it difficult to adapt to the changing walking environment (Rochester et al., 2008).

Consequently, individuals with gait impairments in PD experience further reductions in gait velocity and stride length, decreased symmetry and coordination of the stride pattern, and increased step time variability while dual-tasking (i.e., talking while walking) (Kelly et al., 2012).

The association of gait impairments with adverse consequences like increased fall risk has motivated research in assessing the motor and cognitive factors that exacerbate or improve walking deficits in persons with PD (Kelly et al., 2012). Consequently, a 
number of studies have investigated secondary tasks that may exacerbate or reduce the risk for falls in individuals with PD. For example, Morris, Iansex, Matyas, and Summers (1996) examined effects of both a set of verbal secondary tasks (sentence repetition) and a cognitive secondary task (reciting days of the week backwards) on gait in individuals with PD and age-matched controls. Results illustrated that, although the control group exhibited a slowing of the walking pattern, the changes were not statistically significant. In contrast, it was found that secondary task performance led to a significant decrement in stride length and stride velocity in the PD group that was proportional to the complexity of the task performed.

O'Shea et al. (2002) investigated the effects of motor versus cognitive secondary tasks on gait in PD and age-matched controls. The PD group had reduced stride length and step velocity than controls at baseline and when engaged in dual task situations, regardless of secondary task.

Rochester, Hetherington, Jones, Nieuwboer, Willems, Kwakkel, and Van Wegen (2004) evaluated the effects of functional activities (cognitive and motor) in the home setting on walking performance in individuals with PD. The performance of secondary tasks resulted in a greater reduction of walking speed and stride length in PD, compared to age-matched controls. However, the cognitive task had greater deleterious effects on gait than the motor task.

Consequently, when individuals with PD attempt dual tasking, their footsteps become short and slow (Galletly \& Brauer, 2005; O'Shea et al., 2002), ground clearance reduced, and they become at an increased risk for falls (Bloem, Hausdorff, Visser, \& Giladi, 2004; Morris et al., 1996). 


\subsection{Gait Hypokinesia in Parkinson's Disease: Velocity and Stride Length}

Walking is a complex activity that requires multi-joint coordination and the ability to sequence joint angles and segments during motion (Rochester et al., 2008; Chiu \& Chou, 2012). In healthy subjects, walking is automated and rhythmic, and limb movements are replicated from stride-to-stride while free walking (Morris, Iansek, Matyas, \& Summers, 1994b).

In PD, gait abnormalities are common and result from a combination of hypokinesia (slowness), rigidity, and deficiencies in posture and balance. (Knuttson, 1972). Gait in PD is often characterized by a shuffling gait with small steps, reduced amplitude of upper limb movements, and a stooped posture (Knuttson, 1972). There can also be problems with initiation of gait, difficulty turning and freezing of gait (Tan, Danoudis, McGinley, \& Morris, 2012). Throughout the progression of the PD, gait abnormalities can lead to an increased fall risk, loss of mobility, and loss of independence (Morris et al., 1994b).

Previous studies have shown that individuals with PD have a reduced gait velocity of $25-40 \%$, and reduced stride length of $15-27 \%$ relative to control participants (Rochester, Hetherington, Jones, Nieuwboer, Willems, Kwakkel, \& Van Wegen, 2005; O’Shea et al., 2002; Morris, Iansek, Matyas, Summers, 1994a; Morris et al., 1994b; Mak, 2013). A number of previous studies have revealed that the regulation of stride length is the fundamental deficit of gait hypokinesia in PD (Morris et al., 1994a b; Mak, 2013). For example, Morris et al. (1994b) investigated stride length, gait velocity, and stride cadence in individuals with gait hypokinesia in PD. In experiments where velocity is not controlled, individuals with PD have a reduced stride length and cadence, and an overall 
reduction in gait velocity relative to control participants. However, when velocity is controlled, individuals with PD have a higher walking cadence and shorter stride length relative to controls (Mak, 2013). The authors suggest that the increase in cadence is a compensatory mechanism for the reduced stride length (Morris et al., 1994b). In general, individuals with PD can more easily regulate gait velocity and cadence, and have problems regulating stride length.

The underlying mechanism responsible for stride length regulation in gait hypokinesia is not well understood. Learned movements, such as walking, require internal cues of the basal ganglia to string the movement sequence together. Growing evidence in PD suggests that the internal cue mechanism is defective, leading to the disordered preparation of submovements in the walking sequence (Morris et al., 1994a). In support of this, a number of investigations have found that when provided with external cues, such as auditory or visual cues, disordered participants can achieve normal movement size (Beradelli, Accornero, Argenta, Meco, \& Manfredi, 1986a; Sheridan, Flowers, \& Humell, 1987; Morris et al., 1994b). For example, Morris et al. (1994b) found that when provided with floor markers, individuals with PD were able achieve normal stride length values. These findings suggest that the step pattern is still intact in individuals with PD and that the problem of stride length regulation is related to difficulty activating the correct stepping response (Morris et al., 1994b).

A number of previous studies have shown that typical gait velocity values of healthy control participants are between 0.88 and 1.36 meters per second. In contrast, individuals with gait hypokinesia in PD demonstrate gait velocity values between 0.56 and 0.85 meters per second (Morris et al., 1994 a b; Maggioni, Veicsteinas, Rampichini, 
Ce, Nemni, Riboldazzi, \& Merati, 2011; Rochester et al., 2005; Knuttson, 1972; Murray, Sepic, Gena, Gardner, \& Downs, 1978). Some of the inconsistency in these gait velocity values may be related to the severity of PD across these previous studies. For example, Murray et al. (1978) demonstrated that PD participants with mild to moderate parkinsonian symptoms had walking speeds of 0.82 to 0.95 meters per second, while more severe participants had walking speeds of approximately 0.67 meters per second. In addition, these studies have shown that typical stride length values of healthy control participants are approximately 1.24 to 1.47 meters, while stride length values of PD participants is approximately 0.75 to 0.92 meters (Morris et al., $1994 \mathrm{a} \mathrm{b}$; Knuttson, 1972).

The effect of changes in walking speed on locomotive parameters such as gait velocity, stride length and cadence has also between investigated (Morris et al., 1994a b; Mak, M., 2013; Maggioni et al., 2011; Murray et al., 1978). The purpose of these studies has primarily been to investigate whether stride length and cadence of disordered participants will approximate normal levels when velocity is controlled or equalized. In general, findings demonstrate that PD participants undershoot or underestimate gait velocity, relative to controls, at all walking speeds. For example, at self-selected fast walking speeds, mean gait velocity is approximately $1.42-1.87$ meters per second for control participants, while PD participants have a mean gait velocity of approximately $0.92-1.35$ meters per second. Maggioni et al. (2011) investigated the maximal speed of control and PD participants and found that control and PD participants achieved mean gait velocities of 5.68 and 4.38 meters per second respectively. This suggests that individuals with PD have the capacity for fast walking, however they are consistently 
slower than control participants. In addition, Morris et al. (1994b) demonstrated similar findings when they had PD and control participants walk at slower than normal gait velocities. Control participants reduced their mean gait velocity to 0.78 meters per second from their normal self-selected speed of 1.08 meters per second (a $28 \%$ reduction), while PD participants reduced their mean gait velocity from 0.75 meters per second to 0.58 meters per second, a $22 \%$ reduction.

In addition, stride length values for faster walking speeds were found to be greater for both PD and control participant groups. However, PD participants made a relatively greater increase in their stride length relative to controls. For example, Morris et al. (1994b) demonstrated that when shifting from normal to fast walking speeds, the mean stride length of the PD participants showed a relative increase of $15 \%$ (from $0.92 \mathrm{~m}$ to $1.06 \mathrm{~m}$ ) while the mean stride length of the control participants only showed a relative increase of $10 \%$ (from $1.3 \mathrm{~m}$ to $1.43 \mathrm{~m}$ ). Although it should be noted that, in terms of the change in absolute stride length values, the PD and control participants showed a fairly similar increase in stride length across the shift from normal to fast walking speeds (PD = $+0.14 \mathrm{~m}$; controls $=+0.13 \mathrm{~m})$.

\subsection{Rationale}

A number of studies have looked at the effects of a concurrent speech task on walking performance in individuals with idiopathic Parkinson's disease (see Kelly et al. (2012) for review). Most of these studies have included serial counting, backward counting or verbal fluency tasks. The use of a monologue or conversational speech task has rarely been examined in these concurrent walking and speech studies. In addition, the 
performance measures obtained from the speech production tasks have typically been limited to measures of language performance rather than measures of speech motor performance (i.e. speech rate) or speech acoustic measures (i.e. speech intensity). This is unfortunate, because a few previous studies have indicated that there can be significant effects on speech intensity during concurrent tasks involving manual activities (Ho et al., 2002, Adams, et al., 2010). Adams et al. (2010) found an increase in the speech intensity of PD participants during a concurrent manual task and proposed the energizing hypothesis to explain this enhancing effect. The potential effect of other concurrent motor activities, such as walking, needs to be examined. Finally, previous studies suggest that attentional demands of a speech task may influence the effect of interlocutor distance on speech intensity in PD (Ho et al., 1999a; Adams et al., 2010). Unfortunately no previous studies have attempted to systematically examine the potential interaction between changes in attention demands (i.e., concurrent task effects) and changes in interlocutor distance.

\subsection{Objectives}

1. To examine conversational speech intensity and speech rate in individuals with Parkinson's disease and controls.

2. To examine the effect of several concurrent walking and non-walking tasks on conversational speech intensity and speech rate in Parkinson's and controls.

3. To examine the effect of different concurrent walking speeds on conversational speech intensity and speech rate in Parkinson's and controls.

4. To examine the effects of different interlocutor distance on conversational speech intensity and speech rate in Parkinson's and controls. 
5. To examine the interaction between different concurrent walking tasks and interlocutor distances on the conversational speech intensity and speech rate of Parkinson's and controls.

6. To examine the effect of concurrent walking tasks and conversational speech on walking speed and stride length in Parkinson's and controls.

7. To examine the combined effects of concurrent walking tasks, interlocutor distances and conversational speech on walking speed and stride length in Parkinson's and controls. 


\section{Chapter 2}

\section{Methods}

\subsection{Participants}

This study included 15 participants $(2 \mathrm{~F}, 13 \mathrm{M})$ between 58 and 80 years old $(\mathrm{M}=72.07)$ diagnosed with mild to moderate idiopathic Parkinson's disease and hypophonia and hypokinetic dysarthria as reported by a neurologist. Participants with PD were patients of neurologist Dr. Mandar Jog at the Movement Disorders Clinic in the Department of Clinical Neurological Sciences at the London Health Sciences Centre (LHSC), and were recruited by Dr. Scott Adams and Cassandra McCaig. Participants with PD were stabilized on their anti-Parkinson medications, and tested approximately one to two hours after the regular self-administration of their medication. Three of the participants with PD were not on anti-Parkinson medication. PD participant demographic information is listed in Table 1 . The study also included 14 age-equivalent healthy control participants (7M, 7F) between 59 and 82 years old. The control participants were recruited from the Retirement Research Association and the Centre for Activity and Aging by Dr. Scott Adams and Cassandra McCaig. The control participants were in good overall health, with an absence of any speech, language, hearing (with the exception of C10, however hearing loss was compensated for by the use of hearing aids) or neurological impairments. 
Table 1. PD Participant Demographic Information

\begin{tabular}{|c|c|c|c|c|c|c|}
\hline Participant & Age & Gender & $\begin{array}{l}\text { Years } \\
\text { since } \\
\text { diagnosis }\end{array}$ & $\begin{array}{l}\text { Mini- } \\
\text { Mental } \\
\text { Score }\end{array}$ & $\begin{array}{l}\text { Parkinson } \\
\text { Medication }\end{array}$ & $\begin{array}{l}\text { Previous } \\
\text { Occupation }\end{array}$ \\
\hline PD1 & 73 & $\mathrm{M}$ & 6 & 29 & Sinemet & $\begin{array}{l}\text { Account } \\
\text { Manager }\end{array}$ \\
\hline PD2 & 80 & M & 2 & 29 & None & Mechanic \\
\hline PD3 & 70 & M & 16 & 30 & Sinemet & Engineer \\
\hline PD4 & 62 & M & 17 & 30 & Sinemet & $\begin{array}{l}\text { Lumber } \\
\text { Yard Sales }\end{array}$ \\
\hline PD5 & 74 & M & 16 & 27 & Sinemet & Printing \\
\hline PD6 & 76 & F & 17 & 30 & Sinemet & Teacher \\
\hline PD7 & 76 & M & 3 & 27 & Sinemet & $\begin{array}{l}\text { High School } \\
\text { Teacher }\end{array}$ \\
\hline PD8 & 74 & M & 17 & 30 & Sinemet & $\begin{array}{l}\text { London Life } \\
\text { Manager }\end{array}$ \\
\hline PD9 & 78 & M & 4 & 29 & Levocarb & Engineer \\
\hline PD10 & 67 & M & 7 & 30 & Sinemet & N/A \\
\hline PD11 & 78 & M & 8 & 22 & Sinemet & Veterinarian \\
\hline PD12 & 75 & F & 11 & 28 & None & Housewife \\
\hline PD13 & 73 & M & 3 & 30 & Sinemet & $\begin{array}{l}\text { Heavy } \\
\text { Equipment } \\
\text { Operator }\end{array}$ \\
\hline PD14 & 58 & M & 1 & 30 & None & Controller \\
\hline PD15 & 63 & M & 3 & 28 & Sinemet & Technician \\
\hline
\end{tabular}

Table 2. Control Participant Demographic Information

\begin{tabular}{cccc}
\hline Participant & Age & Gender & Previous Occupation \\
\hline C1 & 59 & M & Language Consultant \\
C2 & 61 & F & Housewife \\
C3 & 86 & M & Production Methods Engineer \\
C4 & 73 & M & Fanshawe Instructor \\
C5 & 73 & F & N/A \\
C6 & 82 & M & Armed Forces, Music \\
& & & Instructor \\
C7 & 76 & F & N/A \\
C8 & 73 & M & High School Teacher \\
C9 & 61 & M & Retired \\
C10 & 66 & F & Social Worker \\
C11 & 65 & F & N/A \\
C12 & 77 & F & Secretary \\
C13 & 80 & F & Nurse \\
C14 & 72 & M & Field Safety Inspector \\
\hline
\end{tabular}


Prior to testing, all participants were given a letter of information (Appendices A and B) about the study, along with a consent form (Appendix C) before agreeing to participate. All participants passed a $40 \mathrm{~dB}$ HL hearing screening (tested at $500 \mathrm{~Hz}, 1000$ $\mathrm{Hz}$, and $2000 \mathrm{~Hz}$ ). None of the participants reported previous history of speech, language, or neurological disorder other than PD. In addition, all PD participants passed a cognitive screening (Mini Mental Status Examination). This study was approved by the Health Sciences Research Ethics Board at the University of Western Ontario (Appendix D).

\subsection{Apparatus}

Conversational Data Acquisition. Each PD and control participant completed all of the experimental procedures during a single, 60-minute session in the Althouse Gymnasium in Althouse College at the University of Western Ontario. The participant was equipped with an M-audio Microtracker II device, with a DPA 4060 miniature omnidirectional head-mounted microphone to record conversational speech. The microphone was placed approximately $6 \mathrm{~cm}$ from the participant's mouth. The microphone was calibrated by having the participant produce a sustained 'ah' for at least 1 second at $70 \mathrm{~dB}$ using a sound level meter placed $15 \mathrm{~cm}$ from the participant's mouth. This was repeated 3-times, and the average was used.

Walking Data Acquisition. Two stationary video cameras were used to record each participant's walking performance. One camera was placed parallel to the participant's walking path, and a second video camera was placed perpendicular to the 
participant's walking path. The video recordings were used to measure walking speed (distance/time) and stride length (distance/number of steps).

\subsection{Experimental Protocol}

The experimental protocol began with the collection of the following supporting information: the Unified Parkinson Disease Rating Scale (Fahn et al., 1987) (PD group only); the Mini Mental Status examination to screen for dementia (PD group only); and an Intake Survey (Appendix E). The participant was then introduced to the walking task where they were familiarized with the walking pathway.

Each participant was asked to perform a variety of separate and concurrent speech and walking tasks for a total of 13 experimental conditions. The order of the 13 conditions was randomized for each participant using an online random sequence generator (Haahr, 1998). The speech tasks involved engaging in a conversation with the experimenter for approximately 2-3 minutes about a familiar topic. The conversational topics included favourite vacations, interests, hobbies, relatives, occupational experiences, etc. The conversations took place with the experimenter positioned at either a one-meter interlocutor distance from the participant or a 6-meter interlocutor distance. The walking tasks involved 5 different tasks. This included 1) sitting, 2) standing, 3) walking at a normal or habitual speed, 4) walking at a speed that is self-perceived to be two times slower than the habitual speed, and 5) walking at a speed that is self-perceived to be two times faster than habitual. The experimental conditions are outlined in Table 3 . 
Table 3. Outline of Experimental Protocol

\begin{tabular}{cccc}
\hline Condition & Walking Task & $\begin{array}{c}\text { Interlocutor } \\
\text { Distance } \\
\text { (meters) }\end{array}$ & $\begin{array}{c}\text { Talking } \\
\text { (YES/NO) }\end{array}$ \\
\hline 1 & Sitting & 1 & YES \\
2 & Sitting & 6 & YES \\
3 & Standing & 1 & YES \\
4 & Standing & 6 & YES \\
5 & Walking Normal & 1 & NO \\
6 & Walking Slow & 1 & NO \\
7 & Walking Fast & 1 & NO \\
\hline 8 & Walking Slow & 1 & YES \\
9 & Walking Slow & 6 & YES \\
10 & Walking Normal & 1 & YES \\
11 & Walking Normal & 6 & YES \\
12 & Walking Fast & 1 & YES \\
13 & Walking Fast & 6 & YES \\
\hline
\end{tabular}

\subsection{Data Measurement}

Conversational Speech Intensity and Rate. All conversational speech recordings were transferred from the M-Audio Microtracker II device to a desktop computer running Praat (Boersma \& Weenink, 2011) software. The acoustic waveform editing and analysis function in the Praat software were used to obtain two primary acoustic measures: average speech rate (words per minute) and average speech intensity (dB SPL). The first 10 conversational utterances (minimum 5 words in length) were analyzed from each experimental condition. The number of words per utterance was divided by the utterance duration (in minutes) in order to obtain a measure of speech rate (words per minute). The estimates of speech rate and speech intensity were based exclusively on continuous (fluent) utterances and did not include pauses greater than 250 milliseconds. 
Walking Speed and Stride Length. To collect a measure of stride length the experimenter observed the perpendicular video recording and manually counted the number of steps in each 21-meter walking segment (end points marked by 2 meter vertical poles) for each walking condition. The number of steps was then divided by the distance of the walking segment (21 meters / \# steps). To collect a measure of walking speed the experimenter would time the duration of each 21-meter walking segment on the perpendicular video recording using a stopwatch. The recorded time (seconds) was then divided by the distance of the walking segment (21 meters / time in seconds). The participant would typically walk along the 21-meter walking segment 2-3 times per walking condition, so the stride length and walking speed measurements were averaged across these repeated walks for each walking condition. The video recordings were analyzed using VLC media player, which allowed the examiner to zoom in on the video recording at any time. The zoom function was used when the participant would approach the vertical pole to collect the most accurate estimate of walking duration and number of steps.

In addition, participants were asked to start walking approximately 2-3 meters prior to the first vertical pole, and to walk 2-3 meters past the second vertical pole before turning around. This ensured that participants maintained a constant speed while walking along the 21-meter walking segment.

\subsection{Data Analysis}

In order to assess the primary objective of this study investigating the effects of concurrent walking on conversational speech intensity and speech rate, two separate 
three-way ANOVA's were performed with the participant group (PD and control) as the between group factor, and walking task (sitting, standing, walking at a speed perceived by the participant to be two times slower than their normal or habitual speed, walking at a normal or habitual speed, and walking at a speed perceived by the participant to be two times faster than their normal or habitual speed) and interlocutor distance (one-meter and six-meters) as the within group factors.

Each of the significant main effects were obtained in the ANOVAs investigating conversational speech intensity were examined in more detail using Bonferroni-corrected post-hoc t-tests. The first post-hoc t-test investigated the effect of walking speed on conversational speech intensity in both the PD and control groups. A second post-hoc ttest investigated the effect of walking at a normal speed versus sitting or standing on conversational speech intensity in both the PD and control groups.

In order to assess the secondary objective of this study investigating the concurrent task effects on stride length and walking speed, two separate three-way ANOVA's were performed with the participant group (PD and control) as the between group factor, and walking speed conditions (walking slow, walking normal, and walking fast) and talking condition (talking and not-talking) as the within group factors.

To investigate the effect of interlocutor distance on stride length and walking speed, two separate three-way ANOVA's were performed with the participant group (PD and control) as the between group factor, and walking speed conditions (walking slow, walking normal, and walking fast) and interlocutor distance (1-meter and 6-meters) as the within group factors.

Each of the ANOVAs were examined with a significance level of $p=.05$. 


\section{Chapter 3}

\section{Results}

This study investigated the effects of concurrent walking tasks and interlocutor distance on conversational speech intensity and speech rate in individuals with Parkinson's disease, and healthy age-equivalent controls. The primary results of this study will be presented in two main sections based on the two primary variables being assessed: conversational speech intensity, and conversational speech rate. For each of these two variables, the results will be presented with regard to the group effect (PD versus control), the effect of the walking conditions, and the effect of the interlocutor distance conditions.

A secondary analysis examined the effect of talking versus not talking on the walking performance in individuals with PD and the healthy age-equivalent controls. The results of this secondary analysis will be grouped into two main sections that are based on the two walking variables being assessed: walking speed and stride length. For each of these two walking variables, the results will be presented with regard to the group effect (PD versus control), the effect of the walking conditions, and the effect of the interlocutor distance conditions.

\subsection{Conversational Speech Intensity}

One of the primary objectives of this study was to examine the effect of concurrent walking tasks on conversational speech intensity in individuals with Parkinson's disease and healthy age-equivalent controls. A three factor, repeated 
measures ANOVA was performed using participant group as the between groups factor with two levels (control, PD). The two within-group factors included interlocutor distance and the type of walking task. The interlocutor distance factor had two levels (one meter and six meters interlocutor distance). The factor related to the type of walking task had five levels (sitting, standing, walking at a speed perceived by the participant to be two times slower than their habitual walking speed, walking at a habitual walking speed, and walking at a speed perceived by the participant to be two times faster than their habitual walking speed). The results of the three-way ANOVA are presented in separate sections related to the main effects (group, interlocutor distance, walking task), the interactions, and the post-hoc comparisons. The results are summarized in Figures 1 and 2 with associated means and standard deviations listed in Tables 4 and 5. The detailed results of the three-way ANOVA related to speech intensity are presented in Appendix F.

Table 4. Average Conversational Speech Intensity Values by Walking Condition at an Interlocutor Distance of One Meter

\begin{tabular}{ccc}
\hline & Control & PD \\
\hline Sitting & $69.76(2.64)$ & $65.58(3.55)$ \\
Standing & $69.31(2.67)$ & $64.23(3.81)$ \\
Walking Slow & $71.33(2.52)$ & $67.61(4.53)$ \\
Walking Normal & $71.85(2.45)$ & $68.24(3.25)$ \\
Walking Fast & $73.41(2.87)$ & $69.79(3.43)$ \\
\hline
\end{tabular}

*Note: speech intensity levels are in dB SPL. Standard deviations appear in parentheses beside means. 


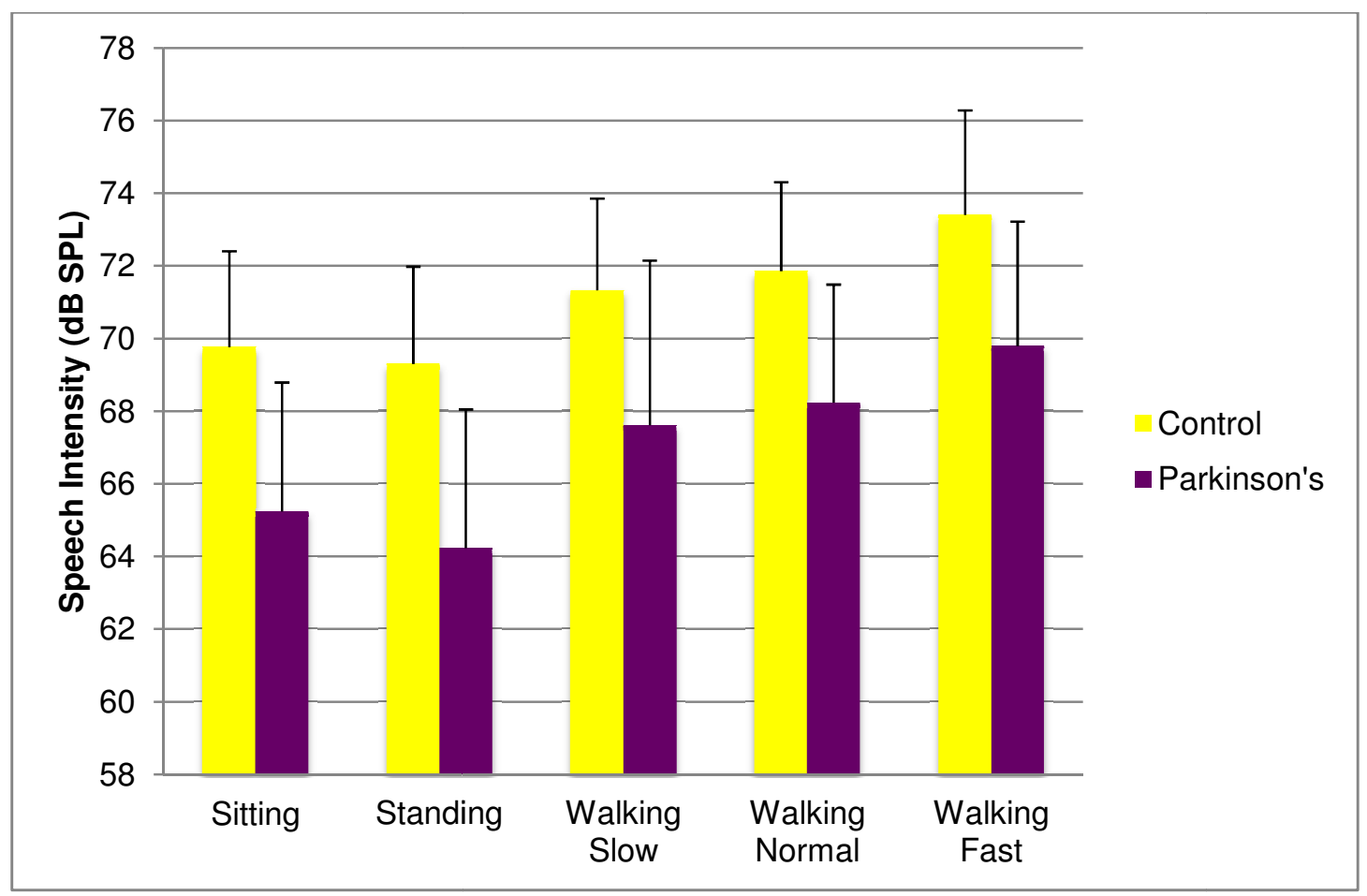

Figure 1. Average conversational speech intensity values by walking condition at an interlocutor distance of one meter. Standard deviations appear as error bars.

Table 5. Average Conversational Speech Intensity Values by Walking Condition at an Interlocutor Distance of Six Meters

\begin{tabular}{ccc}
\hline & Control & PD \\
\hline Sitting & $72.91(2.22)$ & $68.65(3.66)$ \\
Standing & $72.25(2.51)$ & $68.09(3.59)$ \\
Walking Slow & $74.14(2.87)$ & $70.36(3.49)$ \\
Walking Normal & $74.30(2.48)$ & $70.28(3.18)$ \\
Walking Fast & $75.73(3.02)$ & $70.84(3.40)$ \\
\hline
\end{tabular}




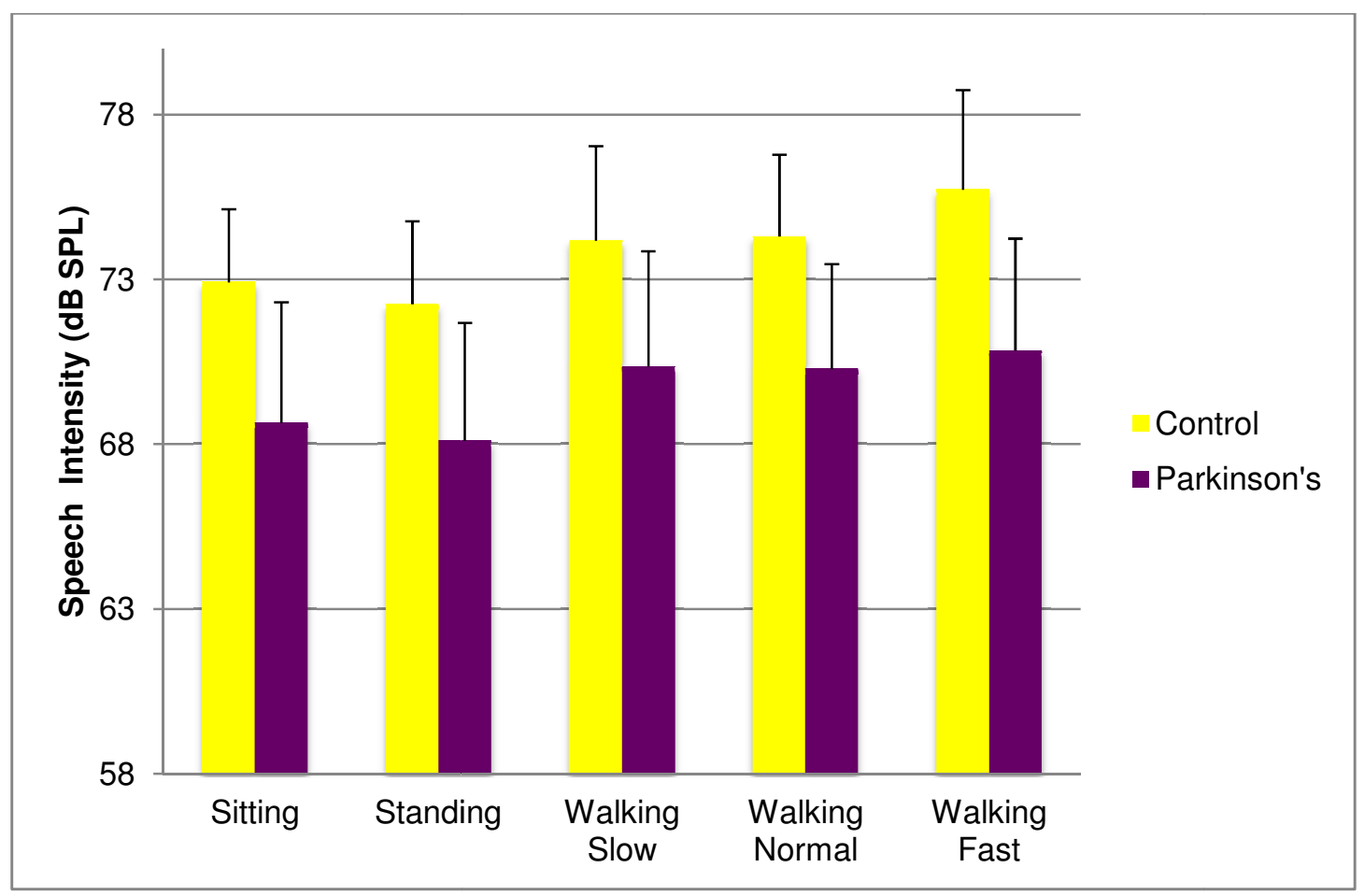

Figure 2. Average conversational speech intensity values by walking condition at an interlocutor distance of six meters. Standard deviations appear as error bars.

\subsubsection{Main Effects: Group, Interlocutor Distance, and Walking Conditions}

The main effect of group was significant $(F(1,21)=11.32, \mathrm{p}=0.003)$ and is illustrated in Figure 3 with associated means and standard error scores listed in Table 6. This significant main effect for group indicates that across all of the experimental conditions the individuals with Parkinson's disease had an overall conversational speech intensity level that was approximately $4.1 \mathrm{~dB}$ lower than the healthy age-equivalent controls. 
Table 6. Average Conversational Speech Intensity Values

\begin{tabular}{cc}
\hline Control & PD \\
\hline 72.31 & 68.17 \\
\hline$(0.85)$ & $(0.89)$
\end{tabular}

*Note: speech intensity levels are in dB SPL. Standard errors appear in parentheses below means.

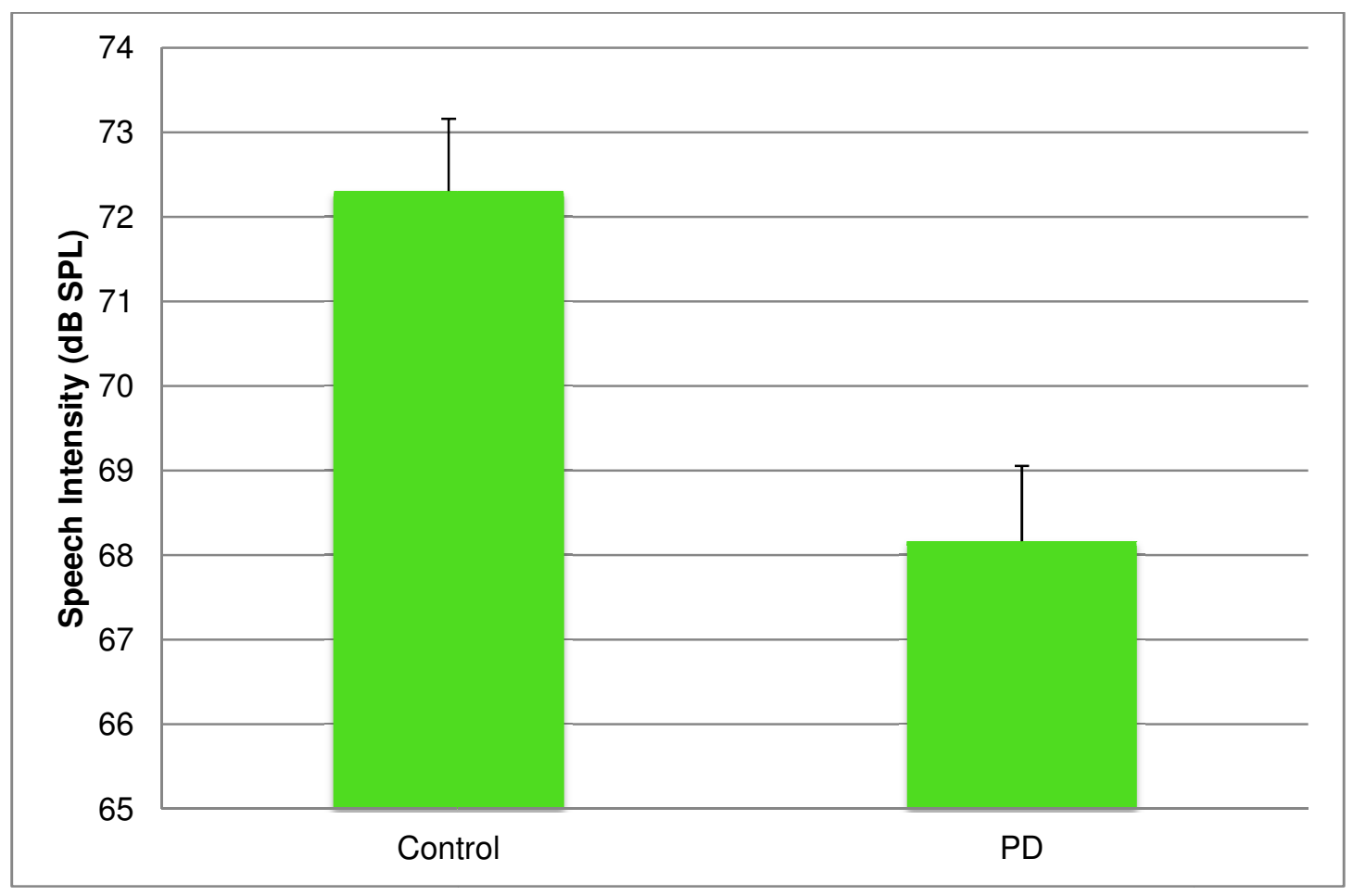

Figure 3. Average conversational speech intensity values. Standard error scores appear as error bars.

The main effect of interlocutor distance was significant $[F(1,31)=103.23, \mathrm{p}=$ 0.000] and is illustrated in Figure 4 with associated means and standard error scores listed in Table 7. This significant main effect for interlocutor distance indicates that both 
participant groups significantly increase conversational speech intensity in response to an increase in interlocutor distance.

Table 7. Average Conversational Speech Intensity of Control and PD Participants at an Interlocutor Distance of One and Six Meters

\begin{tabular}{cc}
\hline 1 meter & 6 meters \\
\hline 69.02 & 71.46 \\
$(0.64)$ & $(0.61)$ \\
\hline
\end{tabular}

*Note: speech intensity levels are in dB SPL. Standard errors appear in parentheses below means.

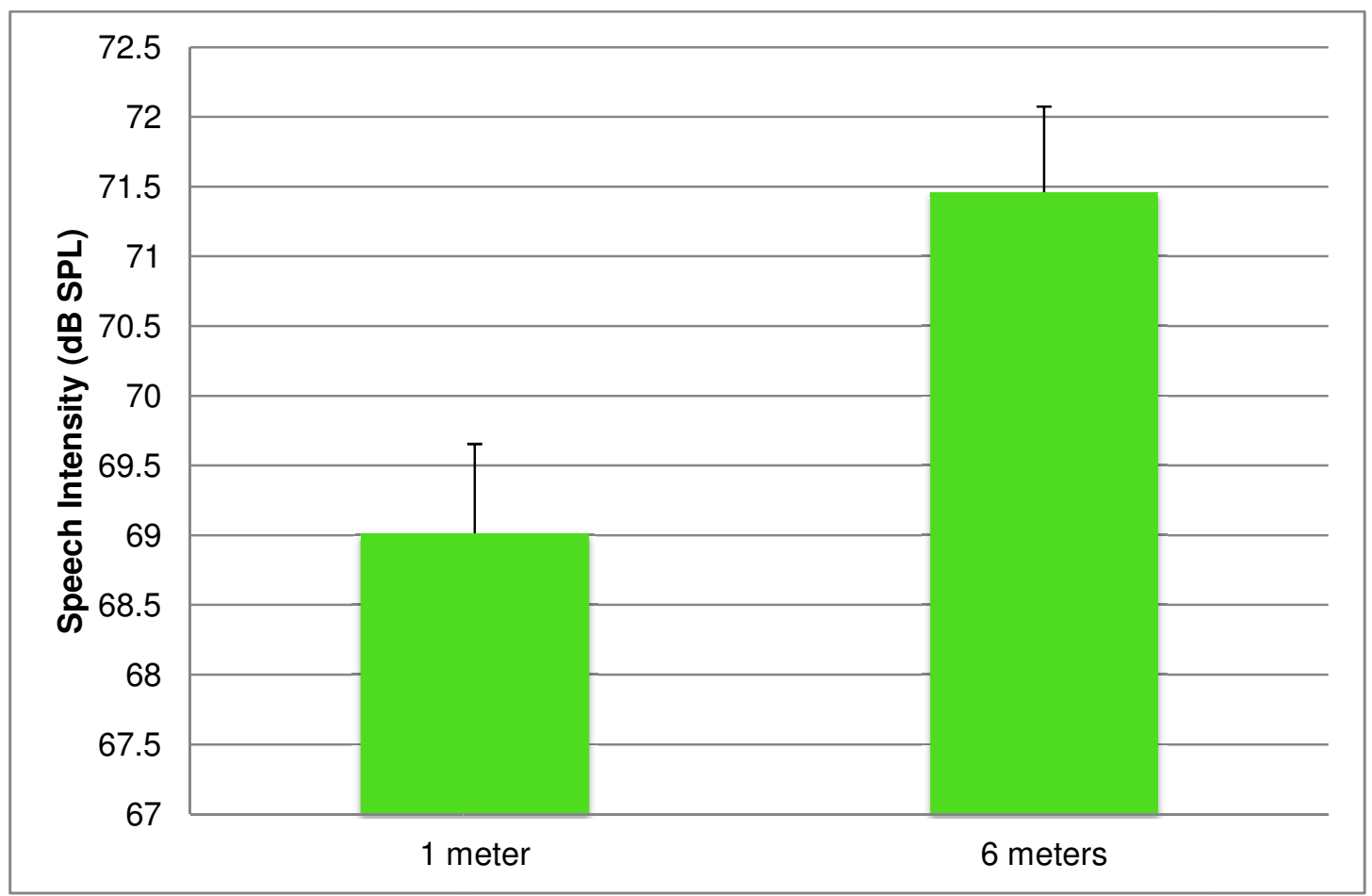

Figure 4. Average conversational speech intensity of control and PD participants at an interlocutor distance of one and six meters. Standard error scores appear as error bars. 
The main effect of the walking task was significant $[F(4,18)=32.90, \mathrm{p}=0.000]$ and is illustrated in Figure 5 with associated means and standard error scores listed in Table 8. This significant main effect of walking task indicates that the type of concurrent walking task significantly effects conversational speech intensity in both participant groups.

Table 8. Average Conversational Speech Intensity Values Across Walking Conditions

\begin{tabular}{ccccc}
\hline Sitting & Standing & Walking Slow & Walking Normal & Walking Fast \\
\hline 69.01 & 68.40 & 70.51 & 71.00 & 72.28 \\
$(0.64)$ & $(0.67)$ & $(0.68)$ & $(0.56)$ & $(0.65)$ \\
\hline *Note: speech intensity levels are in dB SPL. Standard errors appear in parentheses below means.
\end{tabular}

*Note: speech intensity levels are in dB SPL. Standard errors appear in parentheses below means.

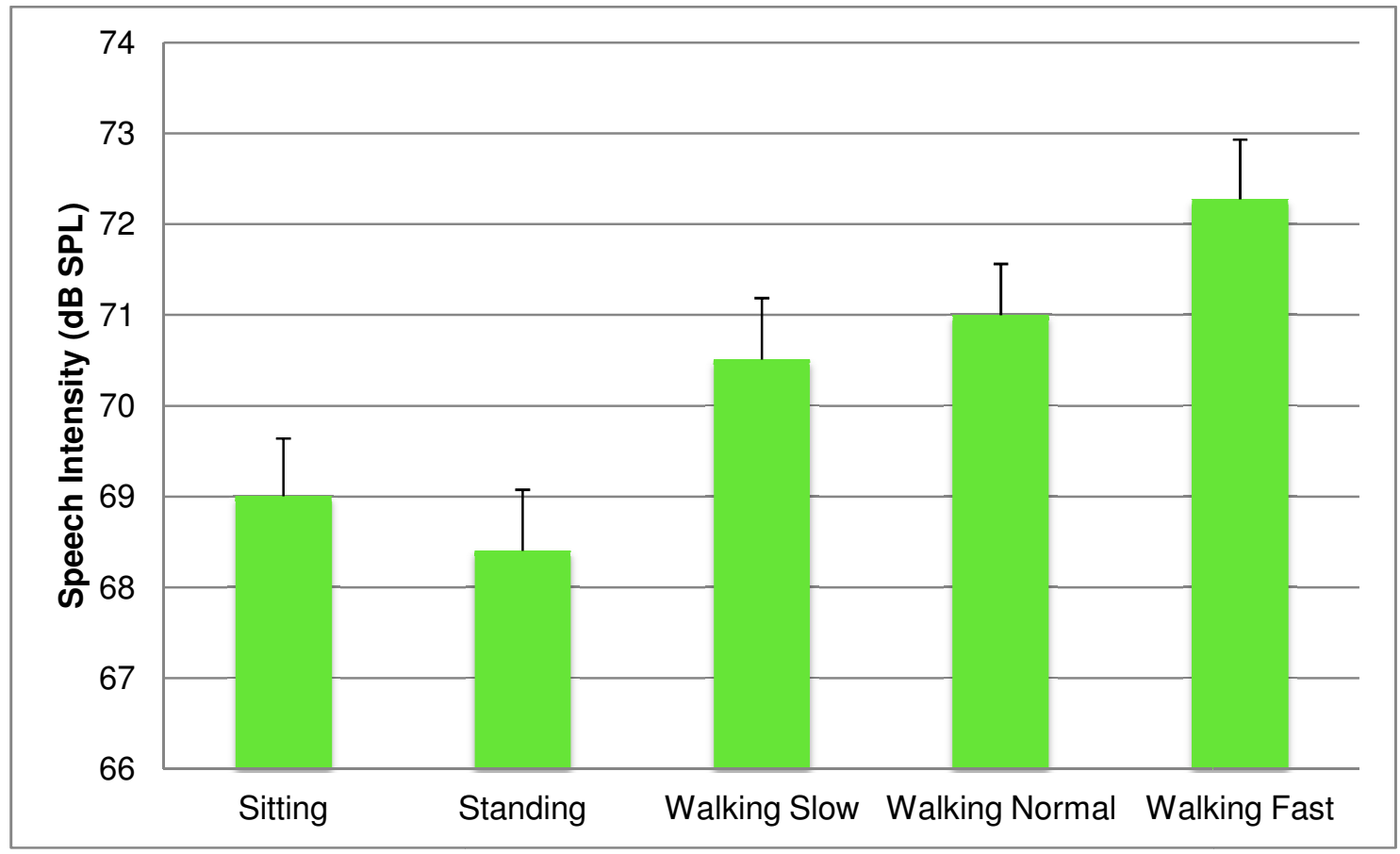

Figure 55. Average conversational speech intensity values across walking conditions. Standard error scores appear as error bars. 


\subsubsection{Interactions Related to Intensity: Group, Interlocutor Distance, and Walking Conditions}

The result for the interlocutor distance by group interaction was not significant $[F(1,21)=0.57, \mathrm{p}=0.460]$. This non-significant result indicates that both the individuals with Parkinson's disease, and the healthy age-equivalent controls had similar changes in conversational speech intensity in response to the change in interlocutor distance. Figure 6 and 7 suggests that as the interlocutor distance increased, the conversational speech intensity levels increased in a similar, parallel manner in both the PD and control groups.

The result for the walking condition by group interaction was not significant $[F(4$, 18) $=0.09, \mathrm{p}=0.959]$. This non-significant result indicates that both the individuals with Parkinson's disease, and the healthy age-equivalent controls had similar changes in conversational speech intensity across the five walking tasks.

The result for the walking condition by interlocutor distance interaction was significant $[F(4,18)=5.73, \mathrm{p}=0.005]$ and is illustrated in Figures 6 and 7 with associated means and standard deviation scores listed in Tables 9 and 10. This result indicates that changes in conversational speech intensity across the five walking conditions are different at an interlocutor distance of one meter than six meters for both participant groups. 
Table 9. Average Conversational Speech Intensity Across Walking Conditions at the One and Six Meter Interlocutor Distances for PD Participants

\begin{tabular}{ccc}
\hline & 1 meter & 6 meters \\
\hline Sitting & $65.58(3.55)$ & $68.65(3.66)$ \\
Standing & $64.23(3.81)$ & $68.09(3.59)$ \\
Walking Slow & $67.61(4.53)$ & $70.36(3.49)$ \\
Walking Normal & $68.24(3.25)$ & $70.28(3.18)$ \\
Walking Fast & $69.79(3.43)$ & $70.84(3.40)$ \\
\hline
\end{tabular}

*Note: speech intensity levels are in dB SPL. Standard deviations appear in parentheses beside means.

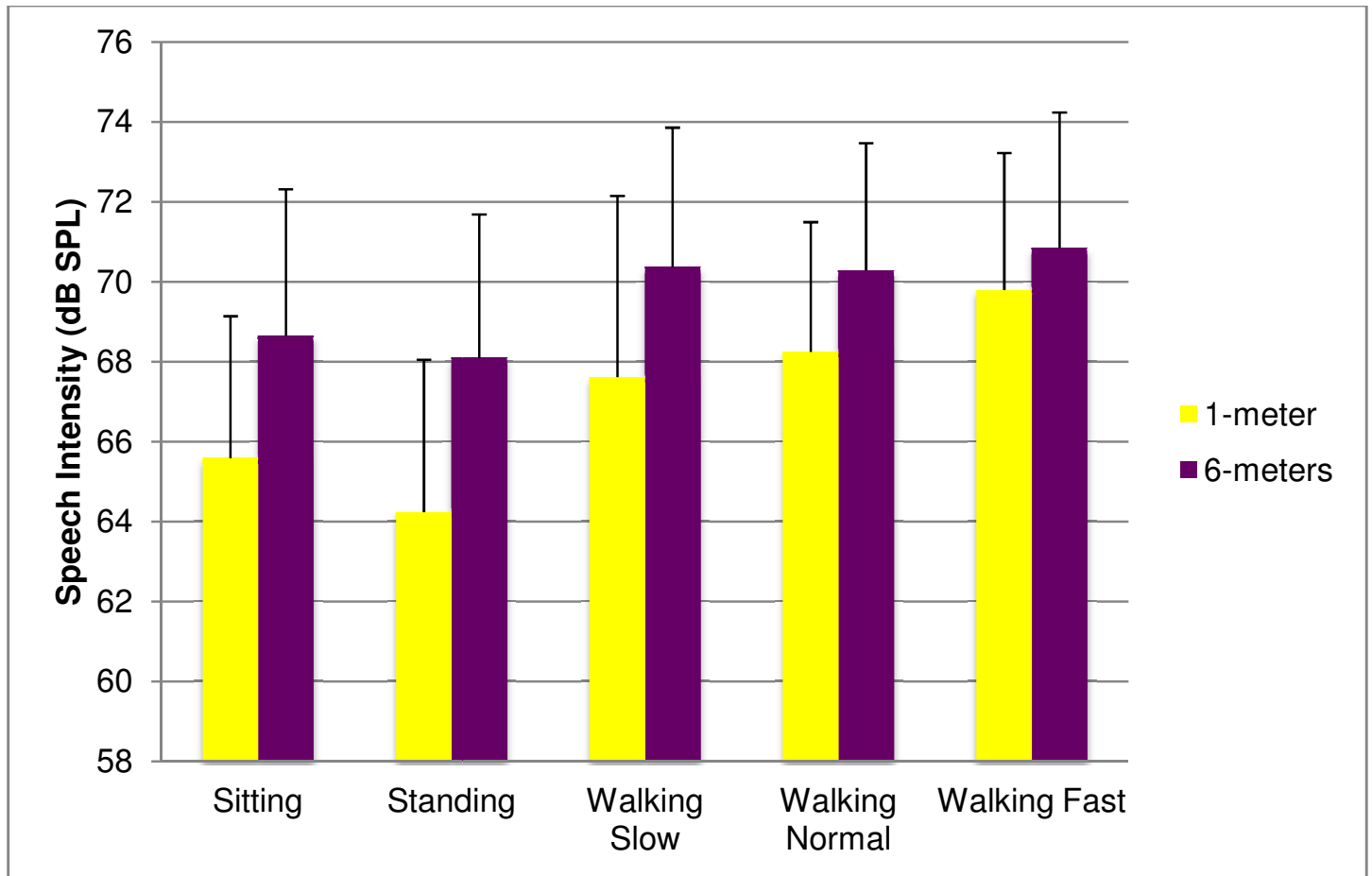

Figure 6. Average conversational speech intensity across walking conditions at the one and six meter interlocutor distances for PD participants. Standard deviations appear as error bars. 
Table 10. Average Conversational Speech Intensity at One and Six Meters Interlocutor Distance Across Walking Conditions for Control Participants

\begin{tabular}{ccc}
\hline & 1 meter & 6 meters \\
\hline Sitting & $69.76(2.64)$ & $72.91(2.21)$ \\
Standing & $69.31(2.67)$ & $72.25(2.51)$ \\
Walking Slow & $71.33(2.52)$ & $74.14(2.87)$ \\
Walking Normal & $71.85(2.45)$ & $74.30(2.48)$ \\
Walking Fast & $73.41(2.87)$ & $75.73(3.02)$ \\
\hline
\end{tabular}

*Note: speech intensity levels are in dB SPL. Standard deviations appear in parentheses beside means.

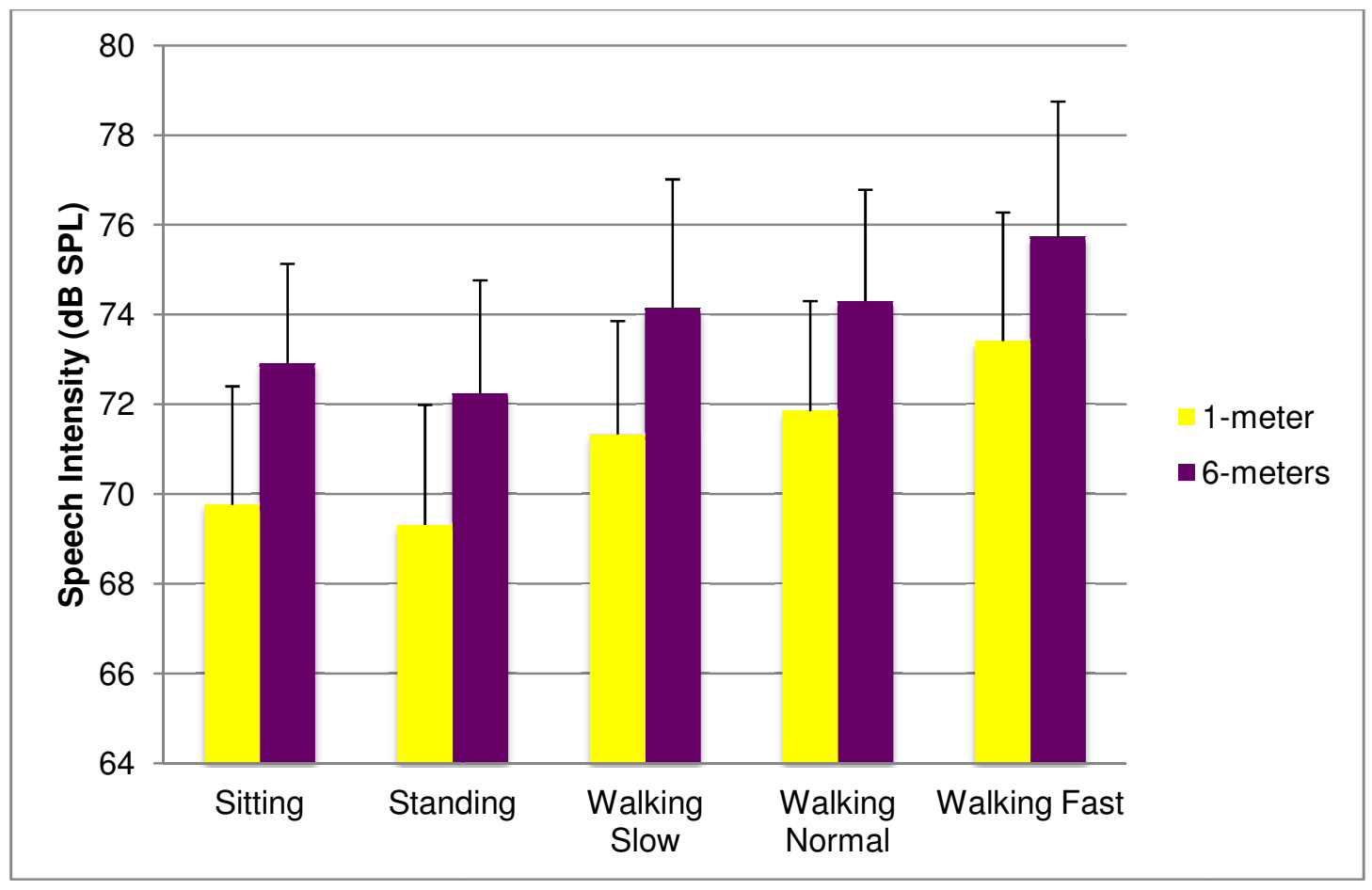

Figure 7. Average conversational speech intensity at one and six meters interlocutor distance across walking conditions for control participants. Standard deviations appear as error bars. 
The three-way interaction involving the group, interlocutor distance, and walking condition was not significant $[F(4,18)=1.65, \mathrm{p}=0.372]$.

\subsubsection{Post Hoc Comparisons: Walking Conditions, Walking Speeds}

In order to examine group differences more closely, a series of post-hoc t-tests were conducted using a Bonferonni correction $(\mathrm{p}=0.05 / 5$ comparisons $=.01)$. The first post-hoc analysis examined within-group differences in conversational speech intensity across three walking speed conditions. Comparisons were made between walking slow and walking fast, walking slow and walking normal, and walking normal and walking fast. The results of this analysis are illustrated in Figures 8 and 9, with associated means and standard deviations listed in Tables 11 and 12. The detailed results of this post-hoc ttest related to walking speed and speech intensity are presented in Appendix G. This analysis indicates that both the PD and control groups had significantly higher speech intensity during the fast walking condition than the normal walking condition when participants were at an interlocutor distance of one meter $([\mathrm{t}(12)=-3.09, \mathrm{p}=.009]$ and $[(t(13)=-4.69, p=.000]$ respectively $)$. At an interlocutor distance of six meters, the control group had a significantly higher speech intensity during the fast walking condition than the normal walking condition $[\mathrm{t}(12)=-3.73, \mathrm{p}=.003]$, but the PD group did not show a significant difference in conversational speech intensity between the fast and normal walking conditions $[\mathrm{t}(13)=-1.42, \mathrm{p}=.177]$. The PD and control groups had significantly higher conversational speech intensity during the fast walking condition than the slow walking condition at an interlocutor distance of one meter $([\mathrm{t}(12)=3.26, \mathrm{p}$ $=.007]$ and $[\mathrm{t}(11)=4.46, \mathrm{p}=.001]$ respectively). At an interlocutor distance of six meters, the control group had a significantly higher speech intensity during the fast 
walking condition than the slow walking condition $[\mathrm{t}(13)=2.95, \mathrm{p}=.011]$, but the PD group did not show a significant difference in conversational speech intensity between the fast and slow walking conditions $[\mathrm{t}(13)=1.59, \mathrm{p}=.136]$. There was no significant difference between the slow walking condition and the normal walking condition for both the PD and control groups at an interlocutor distance of one meter $([\mathrm{t}(14)=0.65, \mathrm{p}=$ $.525]$ and $[\mathrm{t}(11)=1.21, \mathrm{p}=.251]$ respectively $)$ and six meters $([\mathrm{t}(13)=-0.11, \mathrm{p}=.909]$ and $[\mathrm{t}(12)=0.79, \mathrm{p}=.447]$ respectively).

Table 11. Average Conversational Speech Intensity Across Walking Speed Conditions for Control Participants

\begin{tabular}{rccc}
\hline Walking Condition & $\begin{array}{l}\text { Interlocutor } \\
\text { Distance }\end{array}$ & Mean (dB SPL) & $\begin{array}{c}\text { Standard } \\
\text { Deviation }\end{array}$ \\
\hline Walking Slow & 1 meter & 71.33 & 2.52 \\
& 6 meters & 74.17 & 2.84 \\
Walking Normal & 1 meter & 71.85 & 2.45 \\
& 6 meters & 74.30 & 2.48 \\
Walking Fast & 1 meter & 73.41 & 2.87 \\
& 6 meters & 75.45 & 2.95 \\
\hline
\end{tabular}




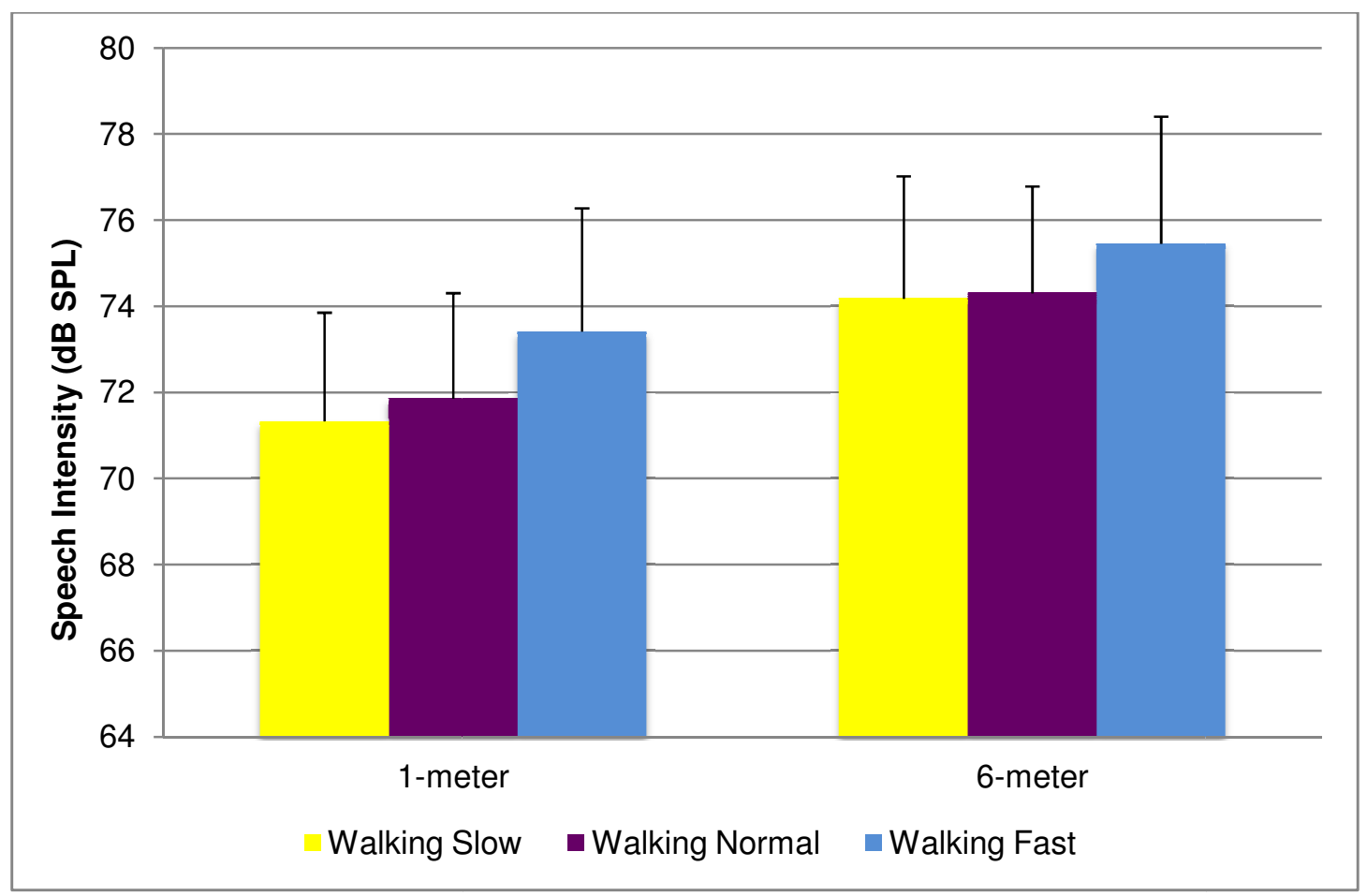

Figure 8. Average conversational speech intensity across walking speed conditions for control participants. Standard deviations appear as error bars.

Table 12. Average Conversational Speech Intensity Across Walking Speed Conditions for PD Participants

\begin{tabular}{lccc}
\hline $\begin{array}{l}\text { Walking } \\
\text { Condition }\end{array}$ & $\begin{array}{l}\text { Interlocutor } \\
\text { Distance }\end{array}$ & Mean (dB SPL) & Standard Deviation \\
\hline Walking Slow & 1 meter & 67.61 & 4.60 \\
& 6 meters & 70.36 & 3.49 \\
\hline Walking Normal & 1 meter & 67.76 & 3.25 \\
& 6 meters & 70.28 & 3.18 \\
\hline Walking Fast & 1 meter & 69.79 & 3.42 \\
\hline & 6 meters & 71.07 & 3.41 \\
\hline
\end{tabular}




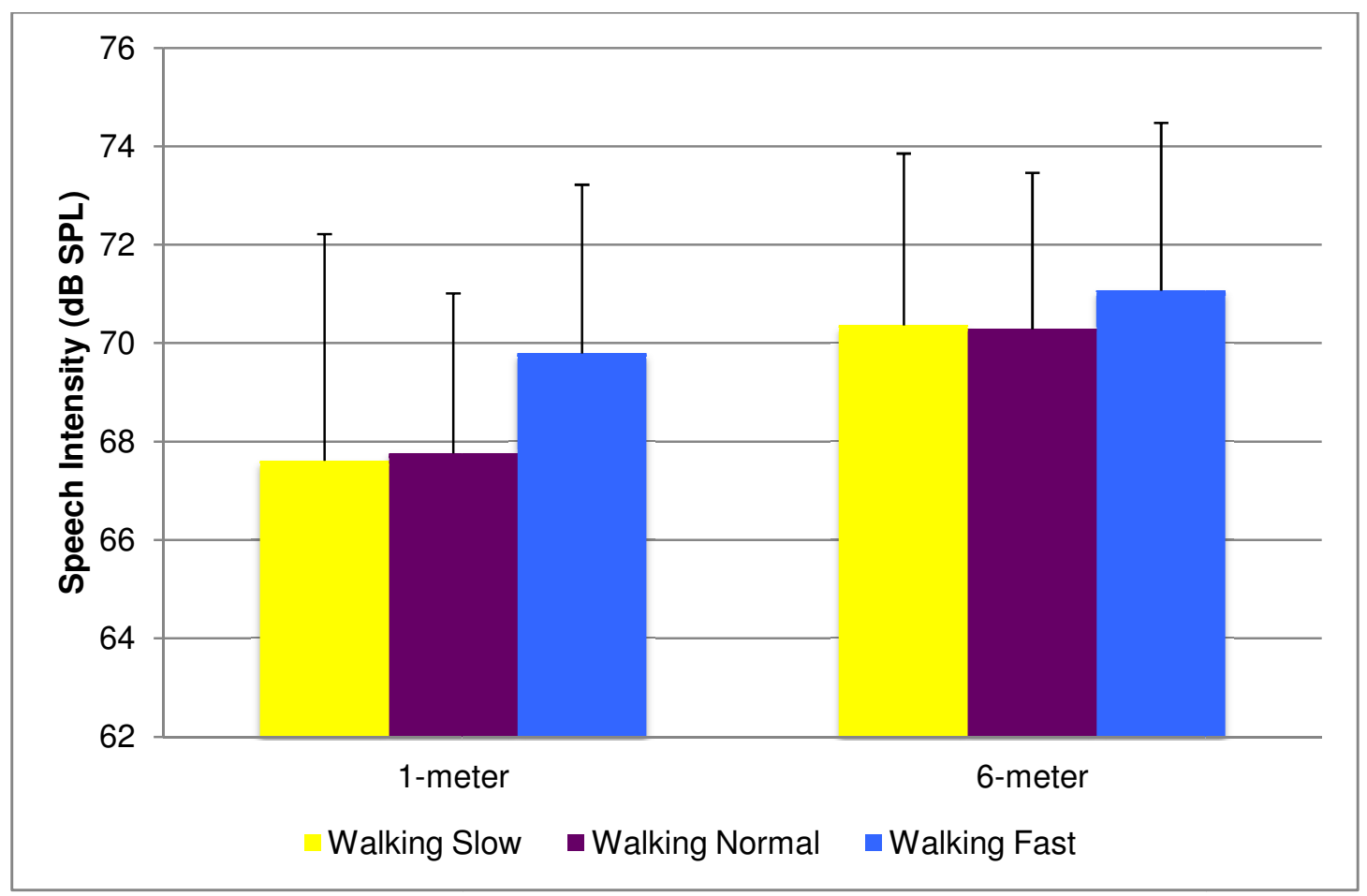

Figure 9. Average conversational speech intensity across walking speed conditions for PD participants. Standard deviations appear as error bars.

A second series of post-hoc t-tests were conducted using a Bonferonni correction $(\mathrm{p}=0.05 / 5$ comparisons $=.01)$ to determine within-group differences in conversational speech intensity between sitting, standing, and walking at a normal or comfortable speed for both the PD and control group as illustrated in Figure 10 and 11, with associated means and standard deviations in Table 13 and 14. This analysis indicates that both the PD and control groups had significantly higher conversational speech intensity while walking at a normal speed than while standing at an interlocutor distance of one meter $([\mathrm{t}(13)=-4.87, \mathrm{p}=.000]$ and $[\mathrm{t}(13)=-7.33, \mathrm{p}=.000]$ respectively $)$ and six meters $([\mathrm{t}(12)$ $=-3.81, \mathrm{p}=.002]$ and $[\mathrm{t}(12)=-5.48, \mathrm{p}=.000]$ respectively). In addition, both the PD and control group had significantly higher conversational speech intensity while walking 
at a normal speed than the sitting condition at an interlocutor distance of one meter $([\mathrm{t}(14)=-3.30, \mathrm{p}=.005]$ and $[\mathrm{t}(13)=-3.71, \mathrm{p}=.003]$ respectively $)$ and six meters $([\mathrm{t}(13)$ $=-2.95, \mathrm{p}=.011]$ and $[\mathrm{t}(12)=-3.39, \mathrm{p}=.005]$ respectively). There was no significant difference in conversational speech intensity for both the PD and control groups between sitting and standing at one meter $([\mathrm{t}(13)=1.68, \mathrm{p}=.116]$ and $[\mathrm{t}(13)=0.80, \mathrm{p}=.435]$ respectively) and six meters $([\mathrm{t}(13)=0.79, \mathrm{p}=.444]$ and $[\mathrm{t}(13)=1.89, \mathrm{p}=.080]$ respectively) interlocutor distance.

Table 13. Average Conversational Speech Intensity in Walking versus Not-Walking Conditions for Control Participants

\begin{tabular}{cccc}
\hline Walking Condition & $\begin{array}{c}\text { Interlocutor } \\
\text { Distance }\end{array}$ & Mean (dB SPL) & Standard Deviation \\
\hline Sitting & 1 meter & 69.76 & 2.64 \\
& 6 meters & 72.91 & 2.30 \\
Standing & 1 meter & 69.30 & 2.68 \\
& 6 meters & 72.25 & 2.51 \\
Walking Normal & 1 meter & 71.85 & 2.45 \\
& 6 meters & 74.30 & 2.48 \\
\hline
\end{tabular}




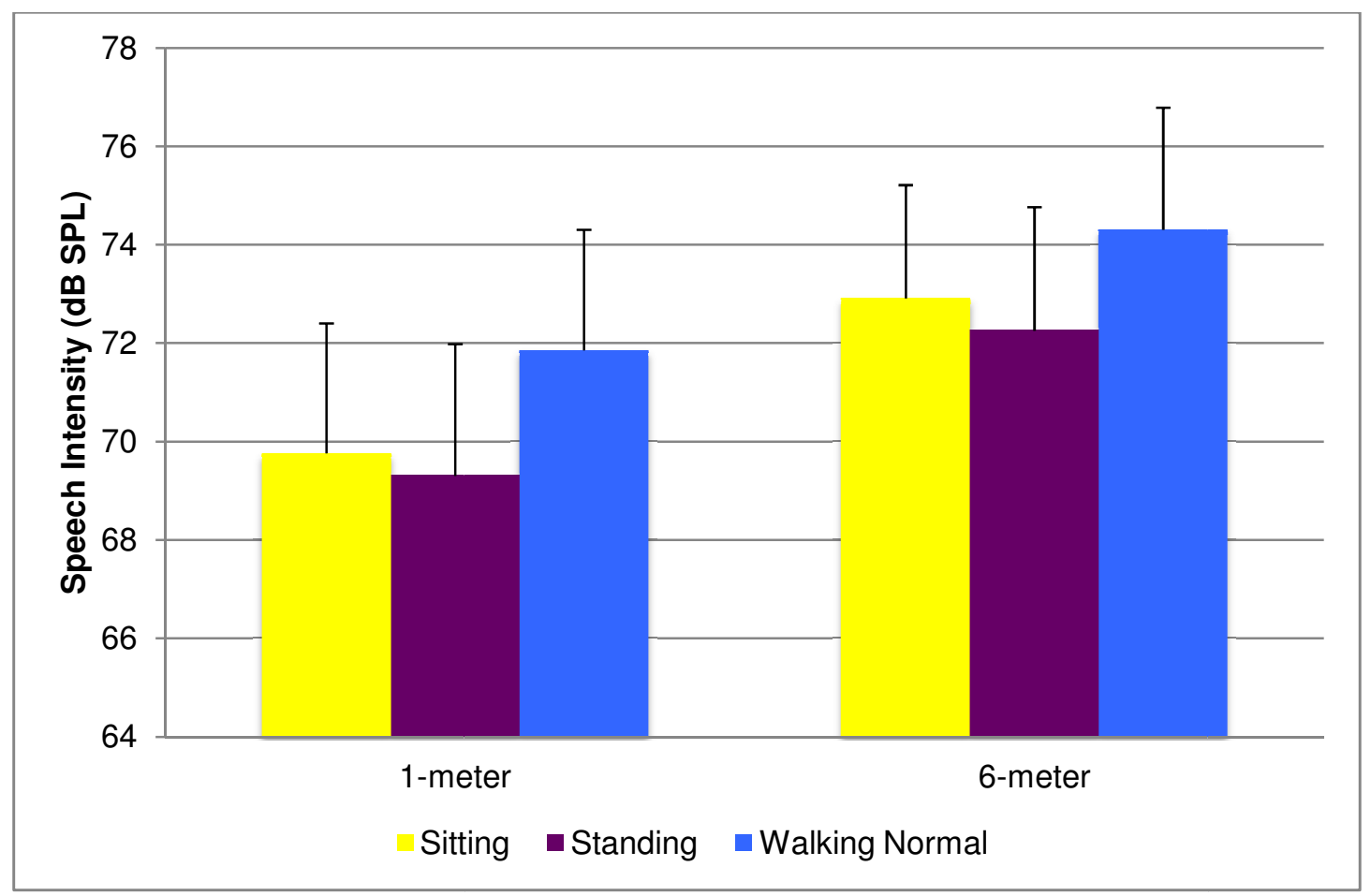

Figure 10. Average conversational speech intensity in walking versus not-walking conditions for control participants. Standard deviations appear as error bars.

Table 14. Average Conversational Speech Intensity in Walking Versus Not-Walking Conditions for PD Participants

\begin{tabular}{cccc}
\hline Walking Condition & $\begin{array}{c}\text { Interlocutor } \\
\text { Distance }\end{array}$ & Mean (dB SPL) & Standard Deviation \\
\hline Sitting & 1 meter & 65.37 & 3.58 \\
& 6 meters & 68.49 & 3.74 \\
Standing & 1 meter & 64.24 & 3.81 \\
& 6 meters & 68.10 & 3.58 \\
\hline Walking Normal & 1 meter & 68.24 & 3.25 \\
& 6 meters & 70.28 & 3.18 \\
\hline
\end{tabular}




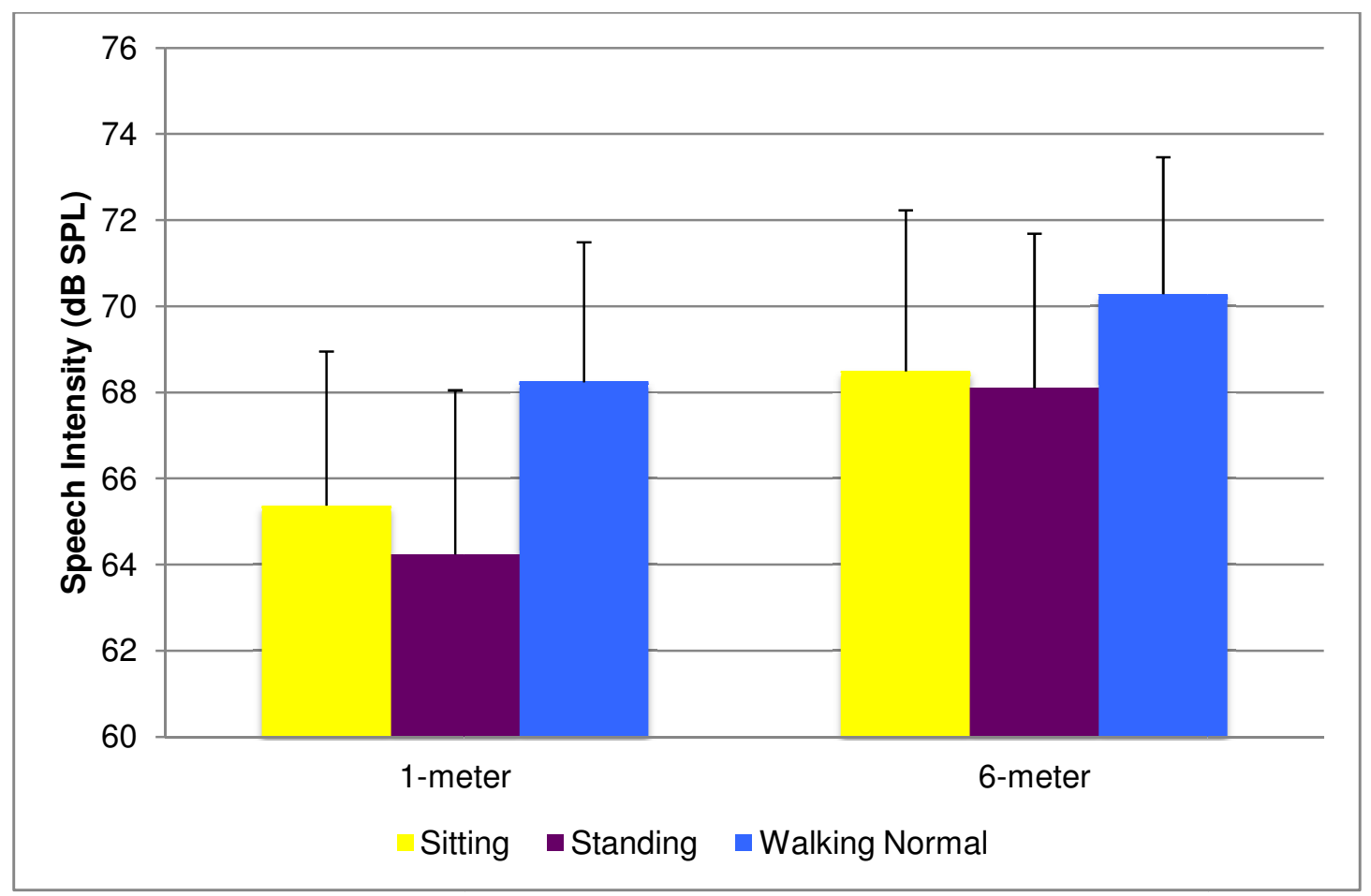

Figure 11. Average conversational speech intensity in walking versus not-walking conditions for PD participants. Standard deviations appear as error bars.

\subsection{Conversational Speech Rate}

The second primary objective of this study was to examine the effect of concurrent walking tasks on conversational speech rate in individuals with Parkinson's disease and healthy age-equivalent controls. A three factor, repeated measures ANOVA was performed using participant group as the between groups factor with two levels (control, PD). The two within group factors included interlocutor distance and the type of walking task. The interlocutor distance factor had two levels (one meter and six meters interlocutor distance). The factor related to the type of walking task had five levels (concurrent speech + sitting, standing, walking at a speed perceived by the participant to be two times slower than their habitual walking speed, walking at a habitual walking 
speed, and walking at a speed perceived by the participant to be two times faster than their habitual walking speed). The results of the three-way ANOVA are presented in separate sections related to the main effects (group, interlocutor distance, walking condition) and the interactions. The results are summarized in Figures 12 and 13 with associated means and standard deviations listed in Tables 15 and 16. The results of this three-way ANOVA related to speech rate are presented in Appendix $\mathrm{H}$.

Table 15. Average Conversational Speech Rate Across Walking Conditions at an Interlocutor Distance of One Meter

\begin{tabular}{ccc}
\hline & Control & PD \\
\hline Sitting & $229.66(29.11)$ & $228.29(38.11)$ \\
Standing & $227.01(23.28)$ & $231.52(36.31)$ \\
Walking Slow & $220.21(34.14)$ & $230.20(47.46)$ \\
Walking Normal & $228.85(31.57)$ & $231.19(38.51)$ \\
Walking Fast & $226.58(20.02)$ & $229.39(43.31)$ \\
\hline
\end{tabular}

*Note: Speech rate values are in words per minute. Standard deviations appear in parentheses beside means. 


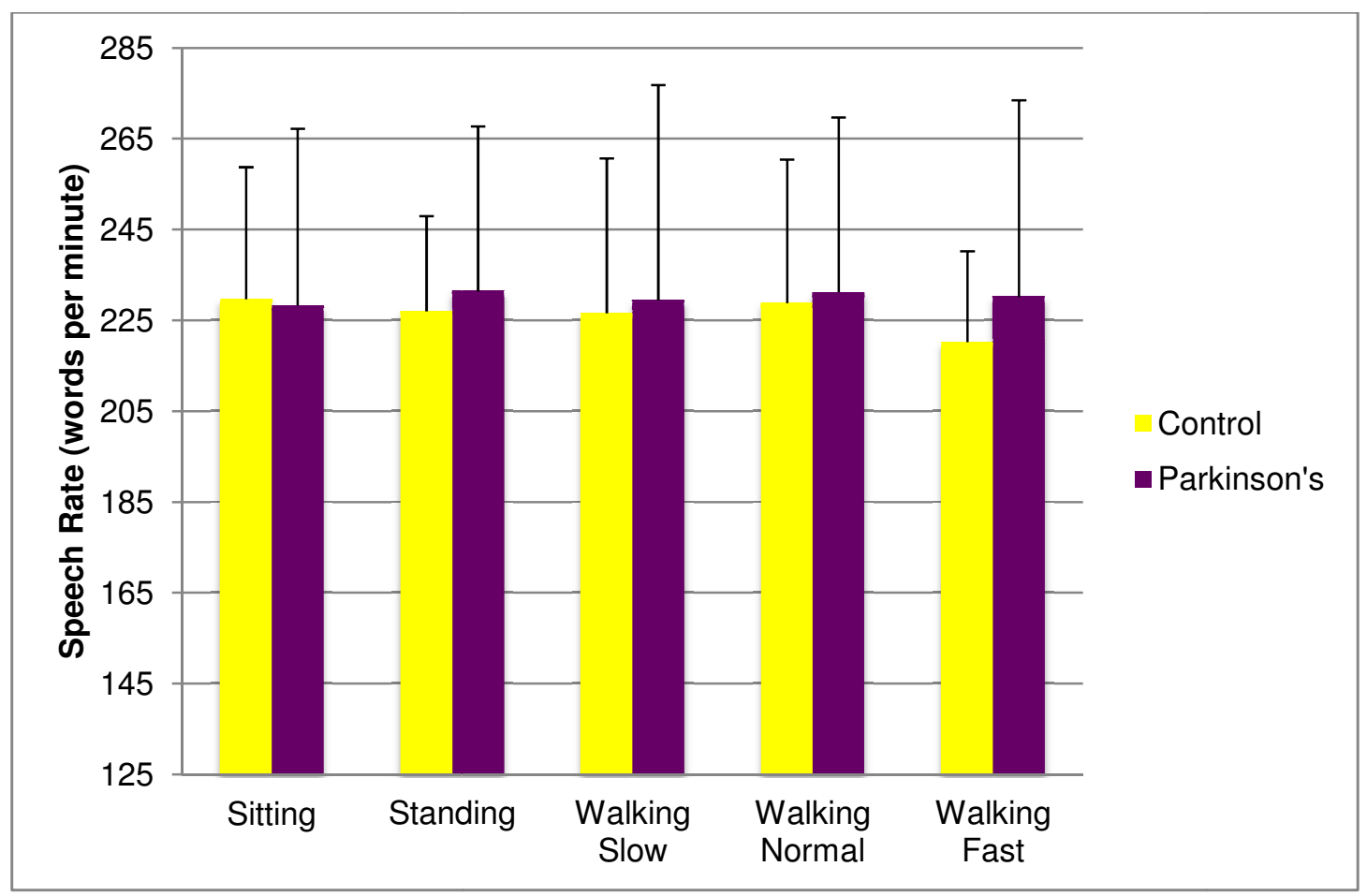

Figure 12. Average conversational speech rate across walking conditions at an interlocutor distance of one meter. Standard deviations appear as error bars.

Table 16. Average Conversational Speech Rate Across Walking Conditions at an Interlocutor Distance of Six Meters

\begin{tabular}{ccc}
\hline & Control & PD \\
\hline Sitting & $218.21(23.28)$ & $232.41(37)$ \\
Standing & $218.89(38.98)$ & $231.60(36.93)$ \\
Walking Slow & $222.55(33.91)$ & $226.65(41.5)$ \\
Walking Normal & $218.16(21.61)$ & $239.97(42.31)$ \\
Walking Fast & $225.31(23.35)$ & $235.53(49.81)$ \\
\hline
\end{tabular}

*Note: speech rate values are in words per minute. Standard deviations appear in parentheses beside means. 


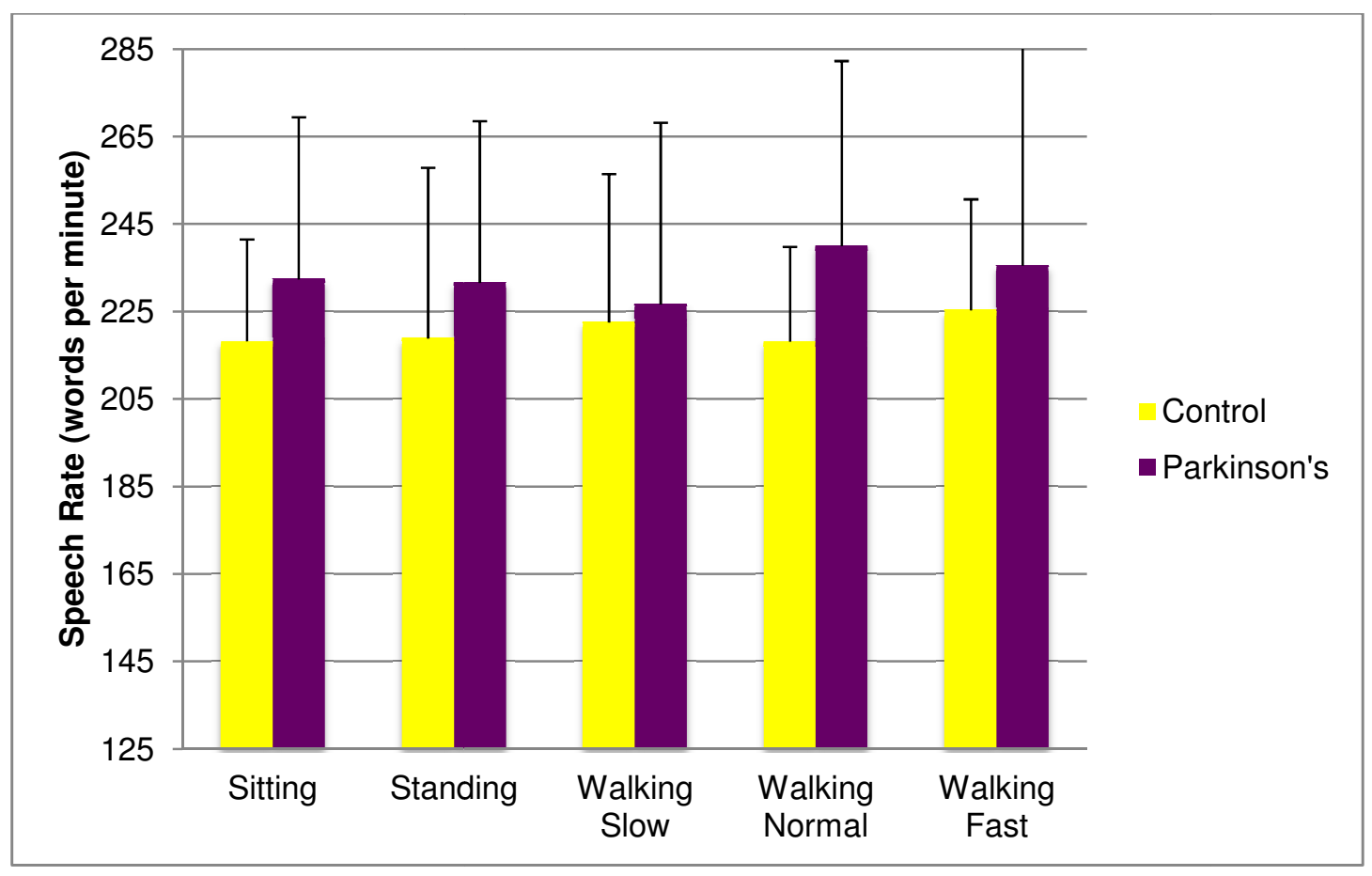

Figure 13. Average conversational speech rate across walking conditions at an interlocutor distance of six meters. Standard deviations appear as error bars.

\subsubsection{Main Effects Related to Speech Rate: Group, Interlocutor Distance, and}

\section{Walking Condition}

The main effect of group was not significant $[F(1,20)=0.41, \mathrm{p}=0.530]$ and is

illustrated in Figure 14 with associated means and standard error scores in Table 17. This non-significant result indicates that there was no overall difference in conversational speech rate between participant groups across all interlocutor distance and walking conditions. 
Table 17. Average Conversational Speech Rate for Control and PD participants

\begin{tabular}{cc}
\hline Control & PD \\
\hline 223.54 & 231.68 \\
$(8.99)$ & $(8.99)$ \\
\hline
\end{tabular}

*Note: Speech rate values are in words per minute. Standard error scores appear in parentheses below means.

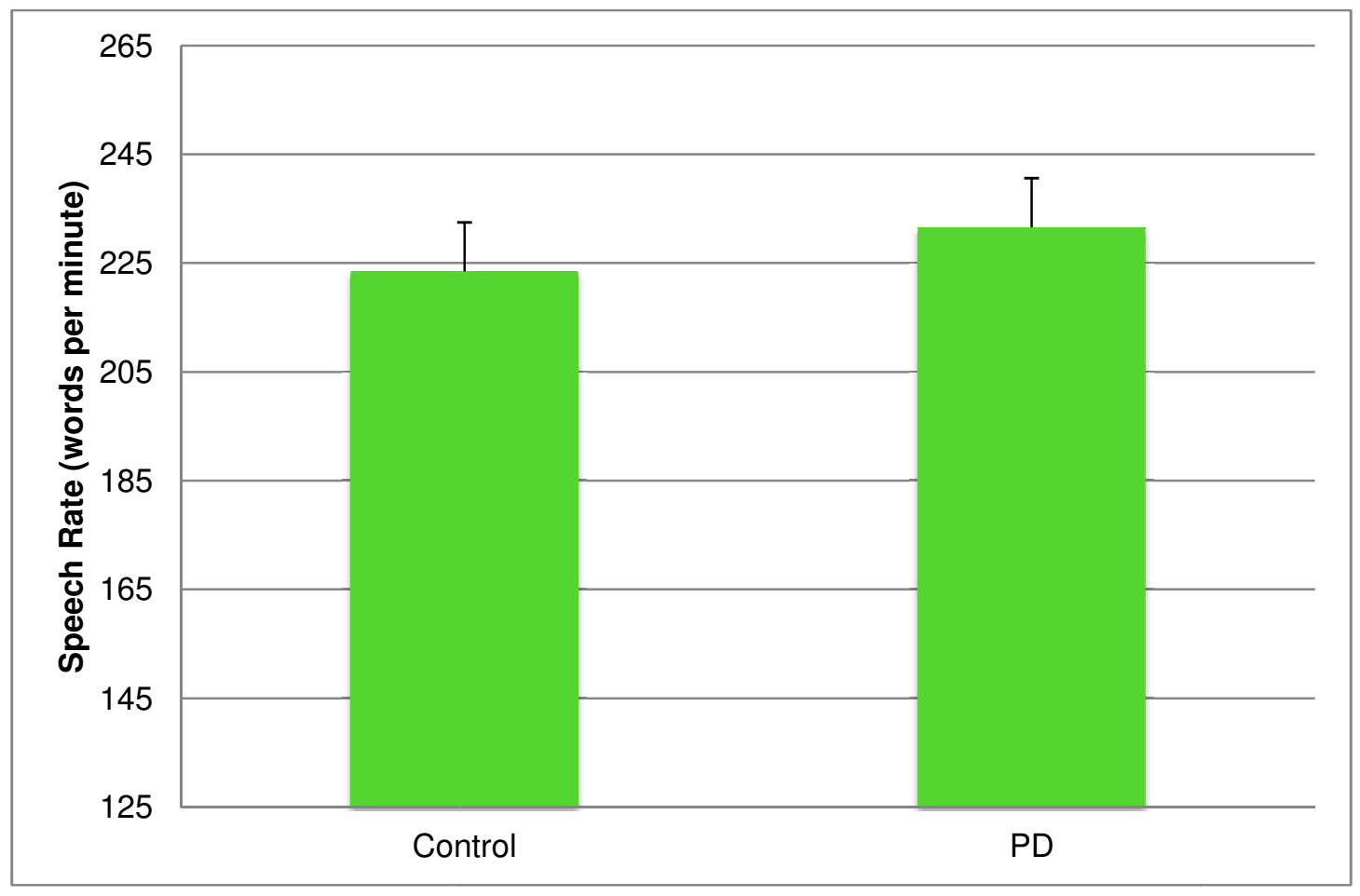

Figure 14. Average conversational speech rate for control and PD participants. Standard error scores appear as error bars.

The main effect of interlocutor distance was not significant $[F(1,20)=0.36, \mathrm{p}=$ 0.555] and is illustrated in Figure 16 with associated means and standard error scores in Table 16. This non-significant result indicates that there was no significant effect of an increase in interlocutor distance on conversational speech rate in both participant groups. 
Table 18. Average Conversational Speech Rate at the One and Six Meter Interlocutor Distances

\begin{tabular}{cc}
\hline 1 meter & 6 meters \\
\hline 228.29 & 226.93 \\
$(6.61)$ & $(6.30)$ \\
\hline
\end{tabular}

*Note: Speech rate values are in words per minute. Standard error scores appear in parentheses below means.

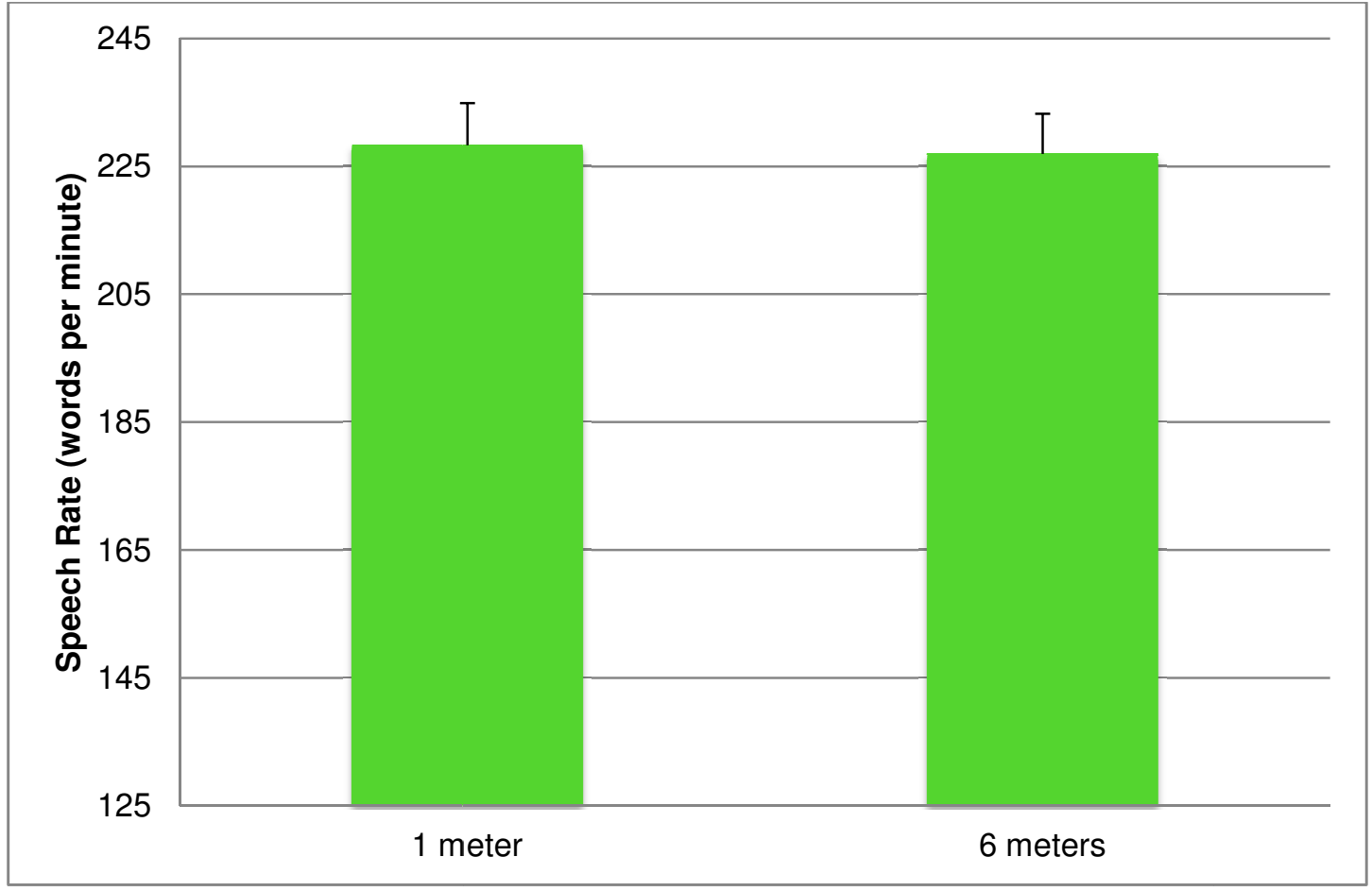

Figure 15. Average conversational speech rate at the one and six meter interlocutor distances. Standard error scores appear as error bars.

The main effect of walking condition was not significant $[F(4,17)=0.49, \mathrm{p}=$ $0.820]$ and is illustrated in Figure 16 with associated means and standard error scores in Table 19. This non-significant result indicates that there was no significant effect of walking condition on conversational speech rate in both participant groups. 
Table 19. Average Conversational Speech Rate Across Walking Conditions

\begin{tabular}{ccccc}
\hline Sitting & Standing & Walking Slow & $\begin{array}{c}\text { Walking } \\
\text { Normal }\end{array}$ & Walking Fast \\
\hline 227.14 & 227.25 & 229.54 & 229.21 & 224.90 \\
$(6.32)$ & $(6.53)$ & $(6.71)$ & $(6.86)$ & $(7.99)$ \\
\hline
\end{tabular}

*Speech rate values are in words per minute. Standard errors appear in parentheses below means.

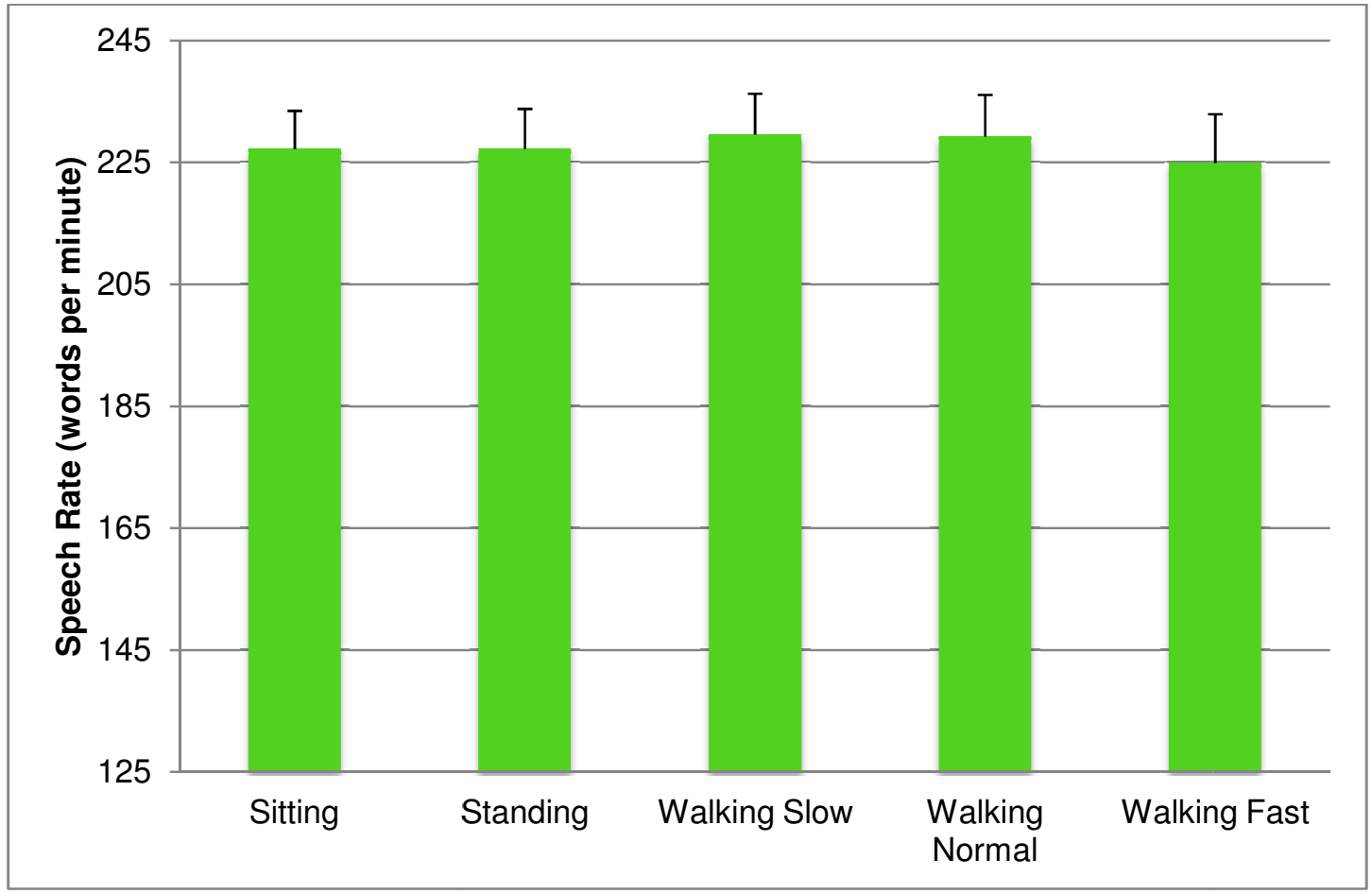

Figure 16. Average conversational speech rate across walking conditions. Standard error scores appear as error bars.

\subsubsection{Interaction Related to Speech Rate: Group, Interlocutor Distance, and}

\section{Walking Conditions}

The result for the interlocutor distance by group interaction was not significant $[F(1,20)=3.87, \mathrm{p}=0.063]$. This non-significant result indicates that both the individuals with Parkinson's disease, and the healthy age-equivalent controls had similar changes in 
conversational speech rate in response to changes in interlocutor distance. While the interaction was not significant, it is noted that there was a tendency for the PD participants to use a slightly faster speech rate as they went from an interlocutor distance of one meter to six meters. On the other hand, there was a tendency for the control participants to use a slightly slower speech rate as they went from an interlocutor distance of one meter to six meters.

The result for walking condition by group interaction was not significant $[F(4,17)$ $=0.30, p=0.960]$. This non-significant result indicates that both the individuals with Parkinson's disease, and the healthy age-equivalent controls had similar changes in conversational speech rate across the five walking conditions.

The result for walking condition by interlocutor distance interaction was not significant $[F(4,17)=0.16, \mathrm{p}=0.958]$. This non-significant result indicates that changes in conversational speech rate across the five walking conditions are similar at both interlocutor distances.

The three way interaction involving group, interlocutor distance, and walking condition was not significant $[F(4,17)=0.46, \mathrm{p}=0.701]$.

\subsection{Walking Speed}

The first secondary objective of this study was to examine the effect of concurrent talking on walking speed in individuals with Parkinson's disease and healthy ageequivalent controls. A three factor, repeated measures ANOVA was performed using participant group as the between groups factor with two levels (control and PD). The two within group factors included the talking condition and the type of walking task. The 
factor related to the talking condition had two levels (talking and not-talking). The factor related to the type of walking task had three levels (walking at a speed perceived by the participant to be two times slower than their habitual walking speed, walking at a habitual walking speed, and walking at a speed perceived by the participant to be two times faster than their habitual walking speed). The results of the three-way ANOVA are presented in separate sections related to the main effects (group, talking condition, and walking condition) and the interactions. The results are summarized in Figures 17 and 18, with associated means and standard deviations listed in Tables 20 and 21. The detailed results of the three-way ANOVA related to walking speed are presented in Appendix I.

Table 20. Average Walking Speed by Walking Condition while Concurrently Talking

\begin{tabular}{ccc}
\hline & Control & PD \\
\hline Walking Slow & $0.83(0.15)$ & $0.75(0.19)$ \\
Walking Normal & $1.26(0.19)$ & $0.94(0.23)$ \\
Walking Fast & $1.69(0.14)$ & $1.24(0.32)$ \\
\hline g speed values are in meters per second. Standard deviations appear in parenthesis beside means.
\end{tabular}




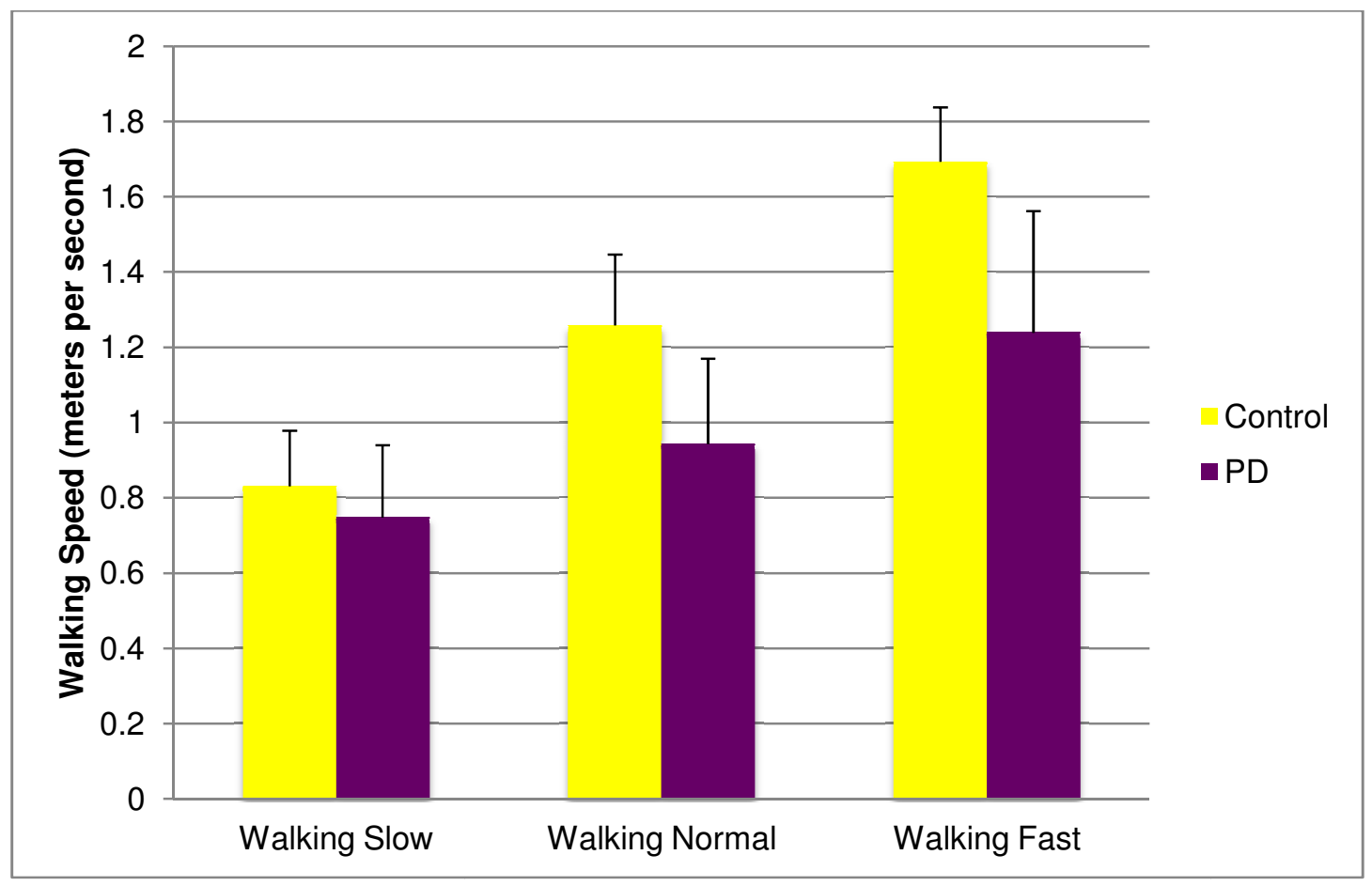

Figure 17. Average walking speed by walking condition while concurrently talking. Standard deviations appear as error bars.

Table 21. Average Walking Speed by Walking Condition while not Talking

\begin{tabular}{ccc}
\hline & Control & PD \\
\hline Walking Slow & $0.85(0.17)$ & $0.866(0.21)$ \\
Walking Normal & $1.41(0.12)$ & $1.149(0.25)$ \\
Walking Fast & $1.84(0.15)$ & $1.459(0.24)$ \\
\hline
\end{tabular}

*Note: Walking speed values are in meters per second. Standard deviations appear in parenthesis beside means. 


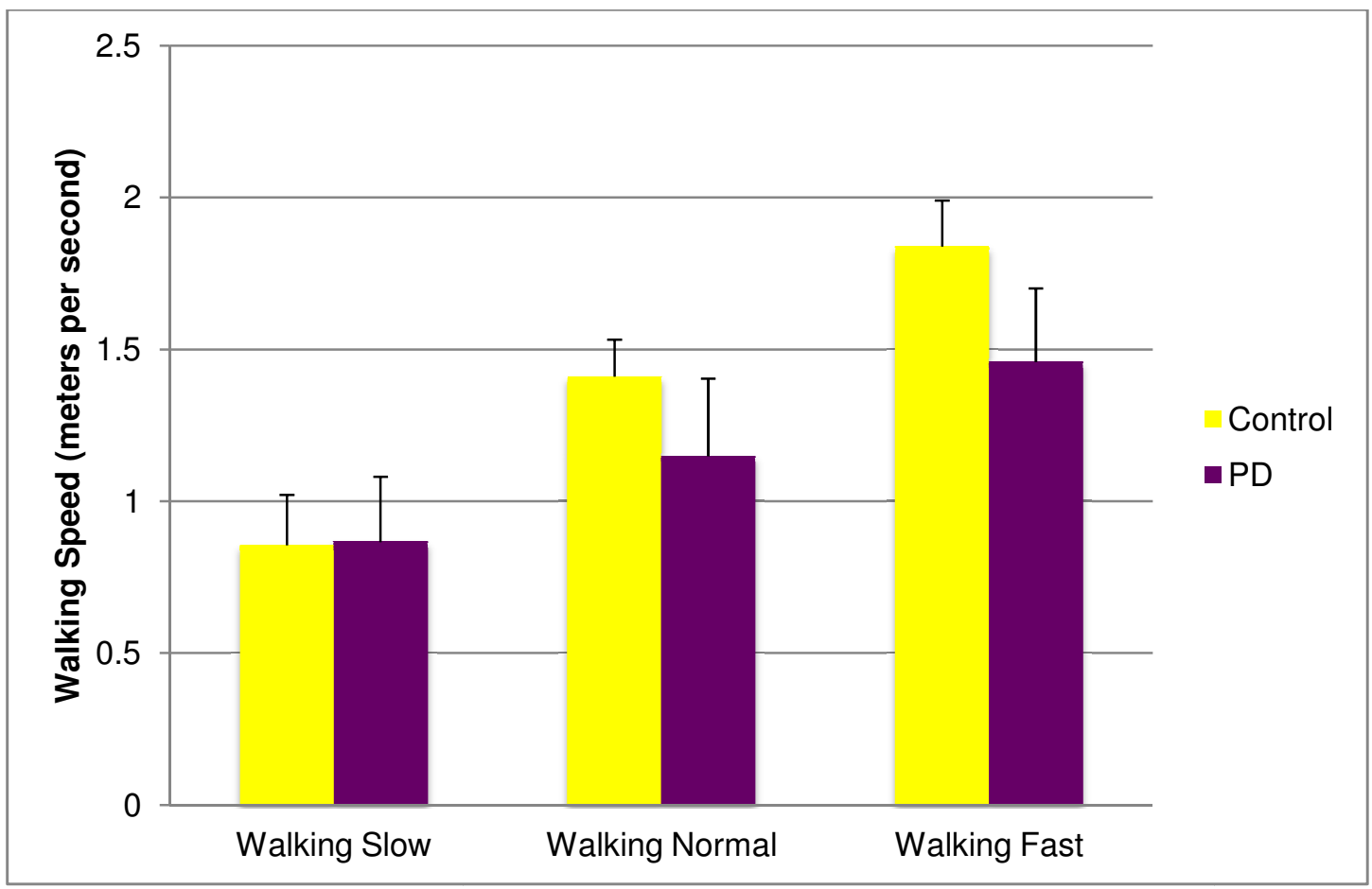

Figure 18. Average walking speed by walking condition while not talking. Standard deviations appear as error bars.

\subsubsection{Main Effects Related to Walking Speed: Group, Talking Condition, and Walking Conditions}

The main effect of group was significant $[F(1,26)=15.18, \mathrm{p}=0.001]$ and is illustrated in Figure 19 with associated means and standard error scores in Table 22. This significant result indicates that there was an overall difference in walking speed between participant groups across all of the talking and not-talking walking conditions. The PD participants had an average walking speed that was about 0.3 meters per second slower than the control participants (about 19\% slower). 
Table 22. Average Walking Speed for Control and PD Participants

\begin{tabular}{cc}
\hline PD & Control \\
\hline 1.07 & 1.31
\end{tabular}

*Walking speed values are in meters per second. Standard error values appear in parenthesis below means.

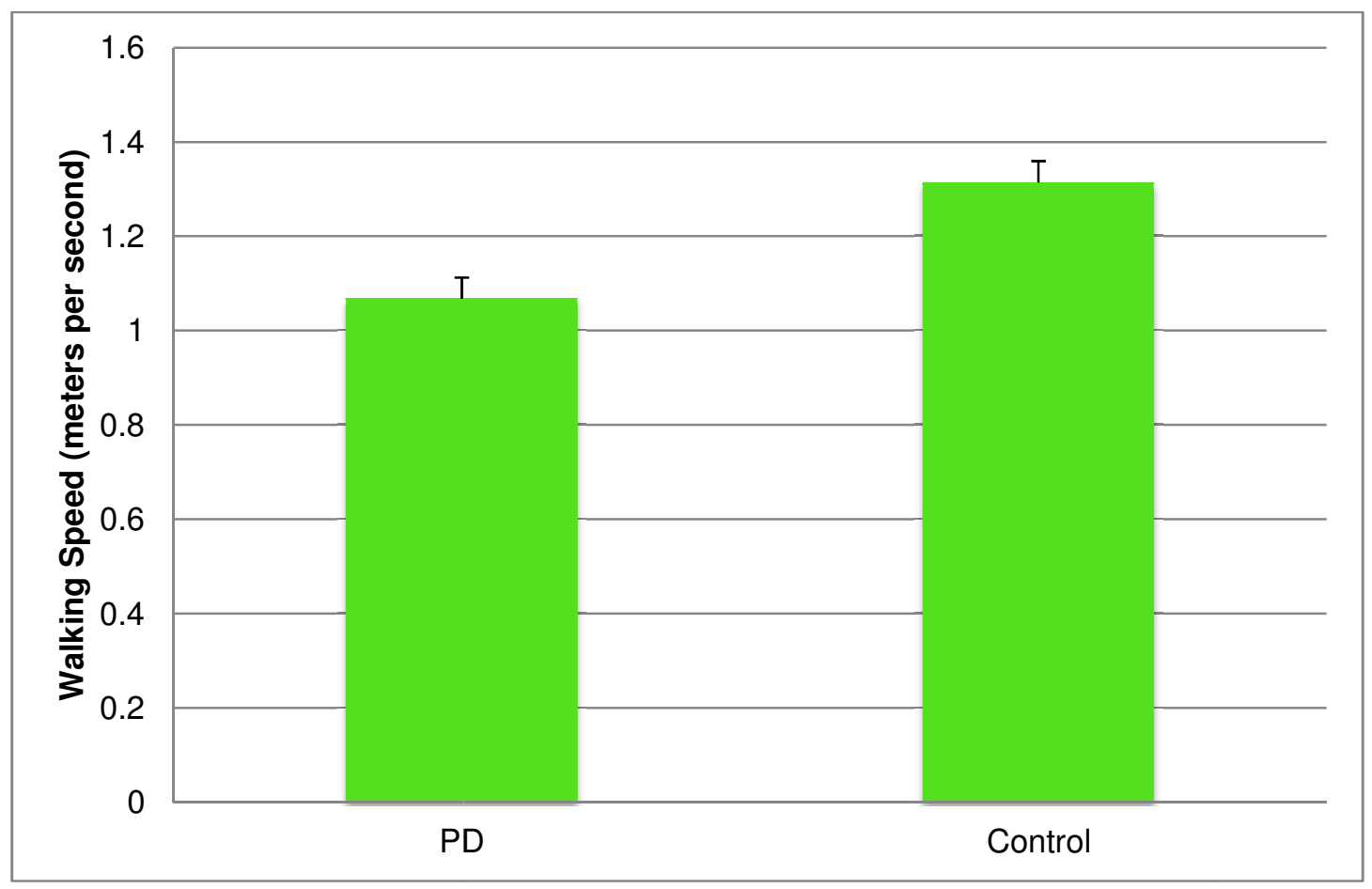

Figure 19. Average walking speed for control and PD participants. Standard error scores appear as error bars.

The main effect of talking was also significant $[F(1,26)=49.35, \mathrm{p}=0.000]$ and is illustrated in Figure 20 with associated means and standard error scores in Table 23. This significant result indicates that both participant groups walked about $11 \%$ slower while concurrently talking and walking, than while walking only. 
Table 23. Average Walking Speed while Concurrently Talking and Walking, and Walking Only

\begin{tabular}{cc}
\hline Talking & Not Talking \\
\hline 1.12 & 1.26 \\
$(0.03)$ & $(0.03)$ \\
\hline
\end{tabular}

*Walking speed values are in meters per second. Standard error scores appear in parenthesis below means.

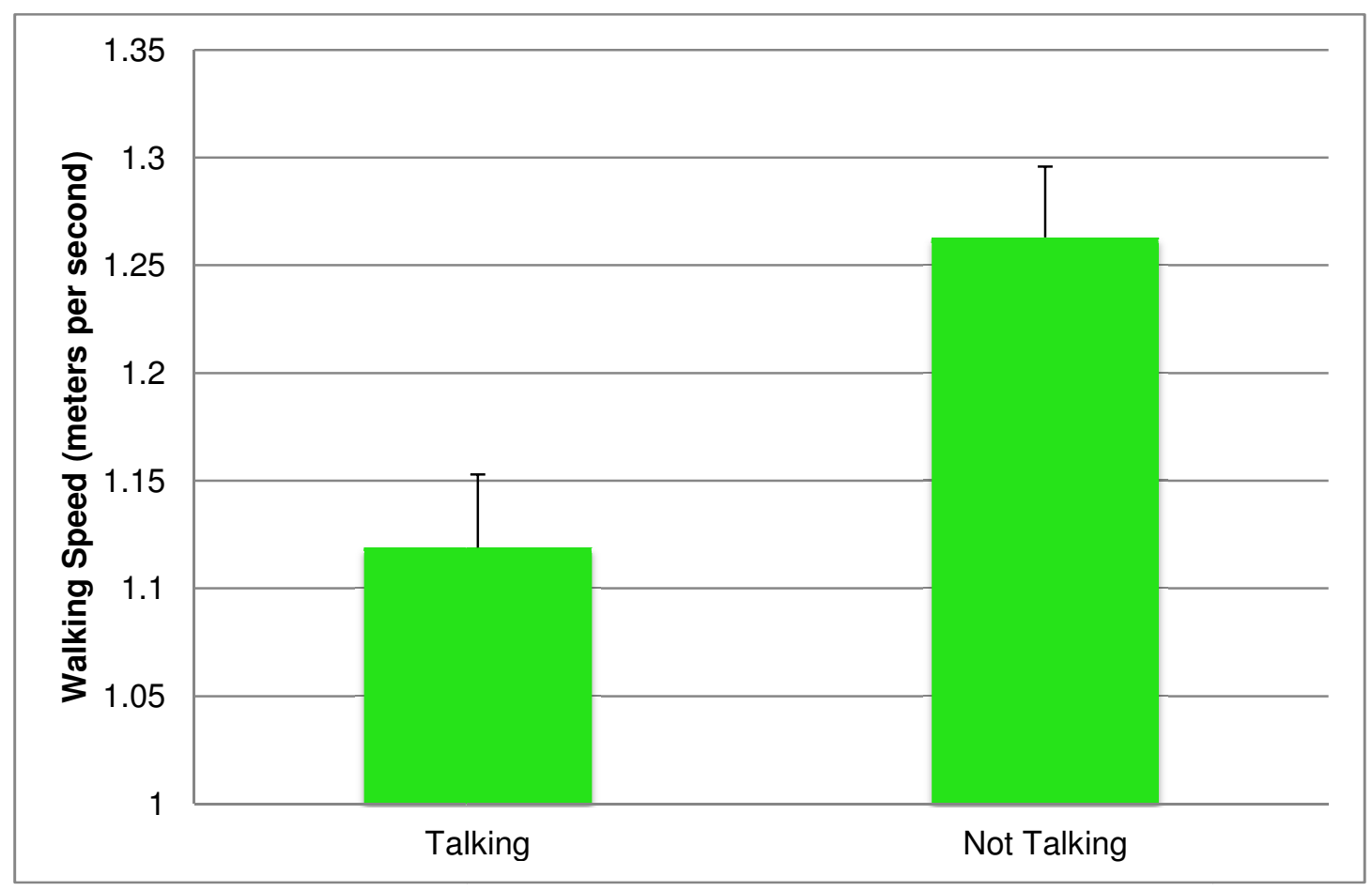

Figure 20. Average walking speed while concurrently talking and walking, and walking only. Standard error scores appear as error bars.

The main effect of walking condition was also significant $[F(2,25)=197.48, \mathrm{p}=$ 0.000] and is illustrated in Figure 21 with associated means and standard error scores listed in Table 24. This significant result verifies that both participant groups appropriately adjusted their walking speed to the walking speed condition. For example, 
all participants walked at their slowest speed in the slow walking condition, and their fastest speed in the fast walking condition.

Table 24. Average Walking Speed Across Walking Speed Conditions for Control and PD Participants

\begin{tabular}{ccc}
\hline Walking Slow & Walking Normal & Walking Fast \\
\hline 0.82 & 1.19 & 1.56 \\
$(0.03)$ & $(0.03)$ & $(0.04)$ \\
\hline
\end{tabular}

*Walking speed values are in meters per second. Standard error scores appear in parenthesis below means.

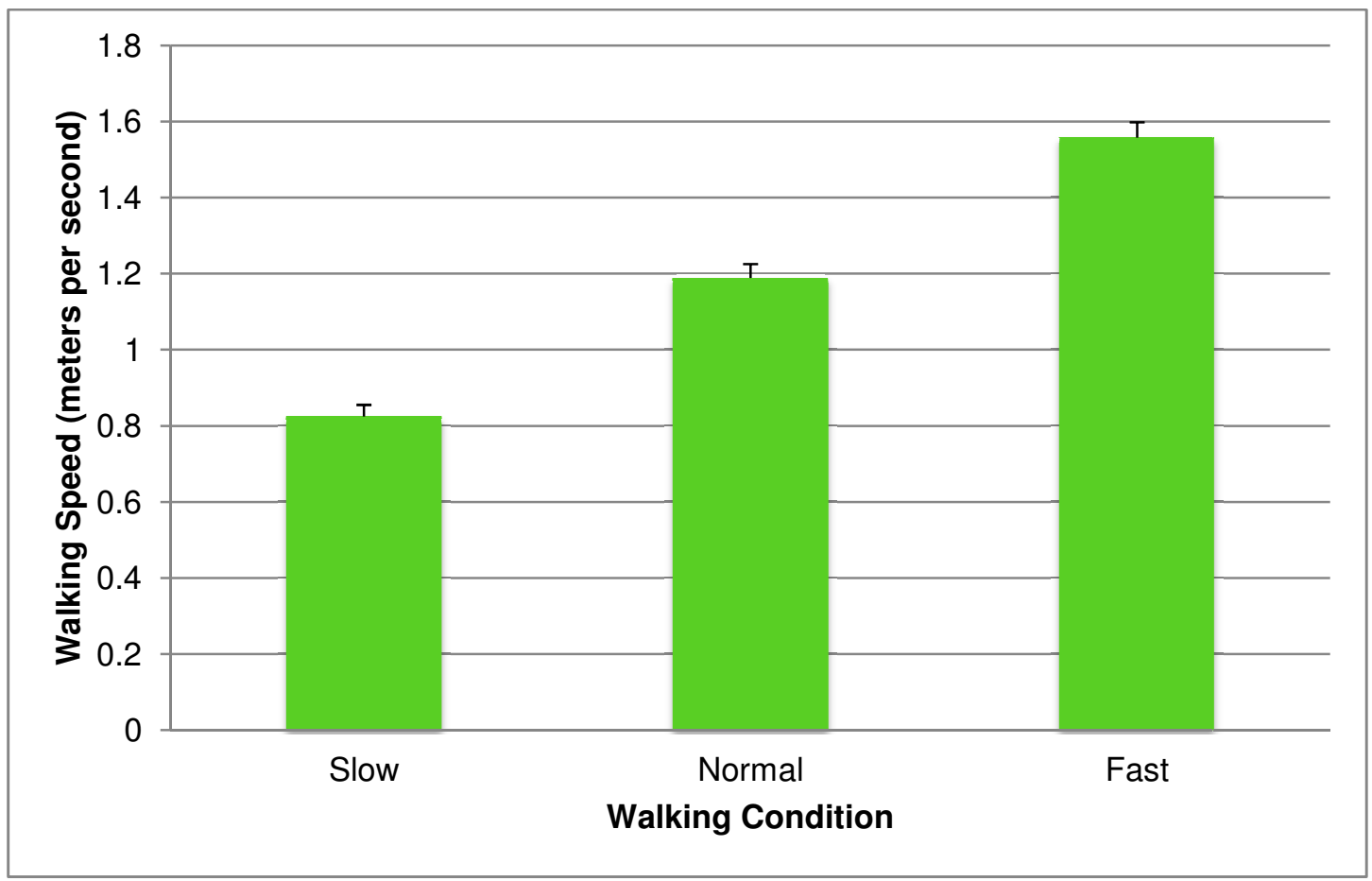

Figure 21. Average walking speed across walking conditions for control and PD participants. Standard error scores appear as error bars. 


\subsubsection{Interactions Related to Walking Speed: Group, Talking Condition, and Walking Conditions}

The result for the walking condition by group interaction was significant $[F(2,26)=15.41, \mathrm{p}=0.000]$. This significant result indicates that the PD participants changed their walking speed in a manner that was different from the way the control participants changed their walking speed. In particular, control participants show relatively greater increases in walking speed than the PD group as the intended walking speed conditions increased from slow to fast (see Figures 19 and 20, with associated means and standard deviations listed in Tables 22 and 23).

The result for the talking condition by group interaction was not significant $[F(1,26)=3.23, \mathrm{p}=0.084]$. This non-significant result indicates that concurrent talking while walking had a similar effect on walking speed in both participant groups.

The result for the walking condition by talking condition interaction was significant $[F(2,25)=8.82, \mathrm{p}=0.001]$. This significant result indicates that some walking conditions may be more affected by talking than other walking conditions. In particular, the fast walking condition was associated with a greater difference in walking speed between the talking and not talking conditions. In contrast, the slow walking condition was associated with a relatively small difference in walking speed across the talking and not talking conditions.

The three way interaction involving group, talking condition, and walking condition was not significant $[F(2,25)=0.15, \mathrm{p}=.835]$. 
A second three factor, repeated measures ANOVA was performed to examine whether interlocutor distance had an effect on walking speed in individuals with Parkinson's disease and healthy age-equivalent controls. The participant group was the between groups factor with two levels (control and PD). The two within group factors included interlocutor distance and the type of walking task. The interlocutor distance factor had two levels (one meter and six meters). The factor related to the type of walking task had three levels (walking at a speed perceived by the participant to be two times slower than their habitual walking speed, walking at a habitual walking speed, and walking at a speed perceived by the participant to be two times faster than their habitual walking speed). The results of the three-way ANOVA are presented in separate sections related to the main effects of interlocutor distance and the interactions. These results are summarized in Figures 22 and 23, with associated means and standard deviation scores listed in Tables 25 and 26. The detailed results of the three-way ANOVA related to walking speed are presented in Appendix J.

Table 25. Average Walking Speed by Walking Condition at an Interlocutor Distance of One Meter

\begin{tabular}{ccc}
\hline & Control & PD \\
\cline { 2 - 3 } Walking Slow & $0.84(0.15)$ & $0.75(0.21)$ \\
Walking Normal & $1.27(0.19)$ & $0.95(0.23)$ \\
*Note: Walking speed values are in meters per second. Standard deviations appear in parenthesis beside means.
\end{tabular}




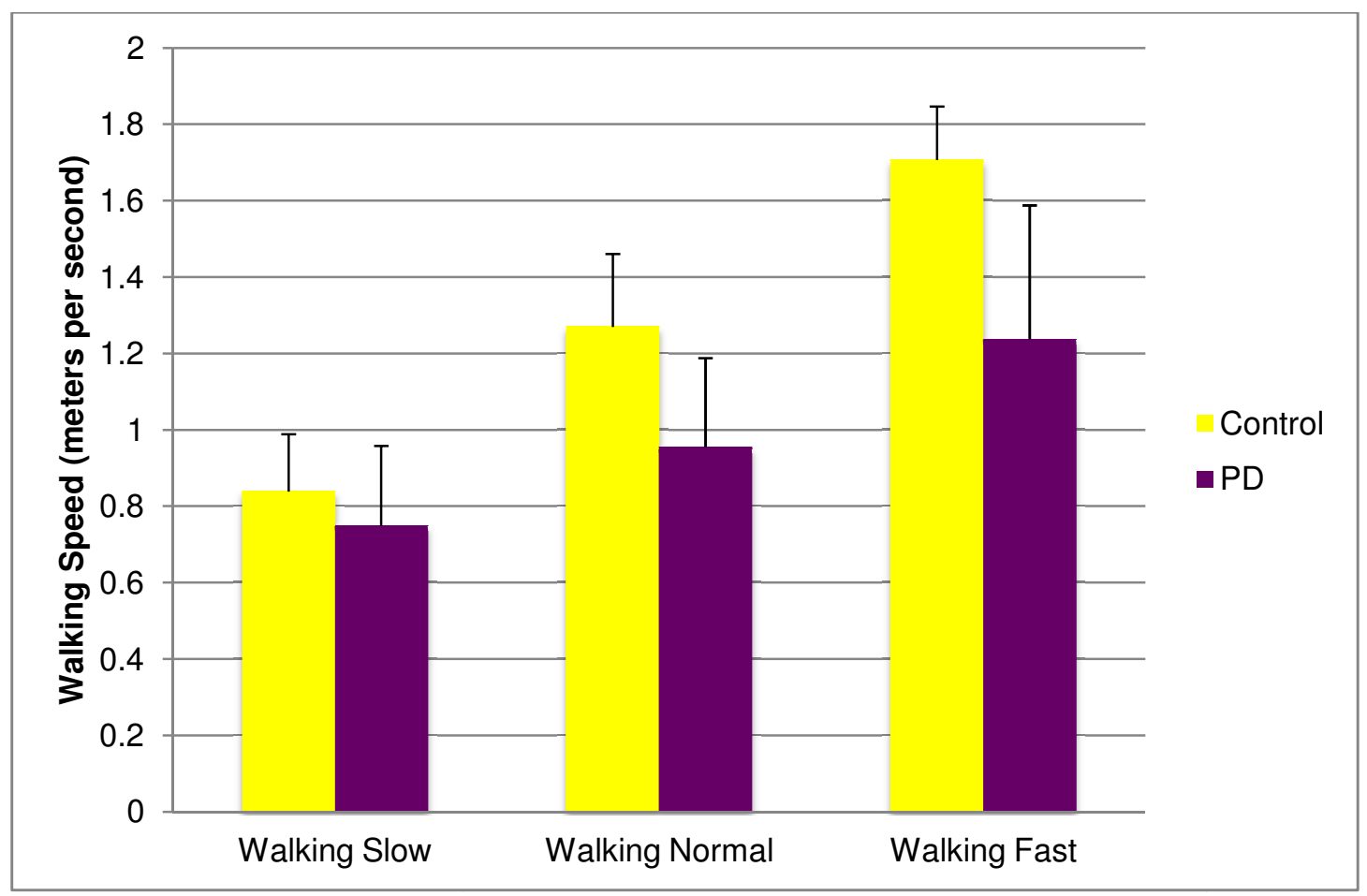

Figure 22. Average walking speed by walking condition at an interlocutor distance of one meter. Standard deviations appear as error bars.

Table 26. Average Walking Speed by Walking Condition at an Interlocutor Distance of Six Meters

\begin{tabular}{ccc}
\hline & Control & PD \\
\hline Walking Slow & $0.85(0.19)$ & $0.722(0.24)$ \\
Walking Normal & $1.24(0.15)$ & $0.937(0.28)$ \\
Walking Fast & $1.65(0.13)$ & $1.261(.37)$ \\
\hline
\end{tabular}

*Note: Walking speed values are in meters per second. Standard deviations appear in parenthesis beside means. 


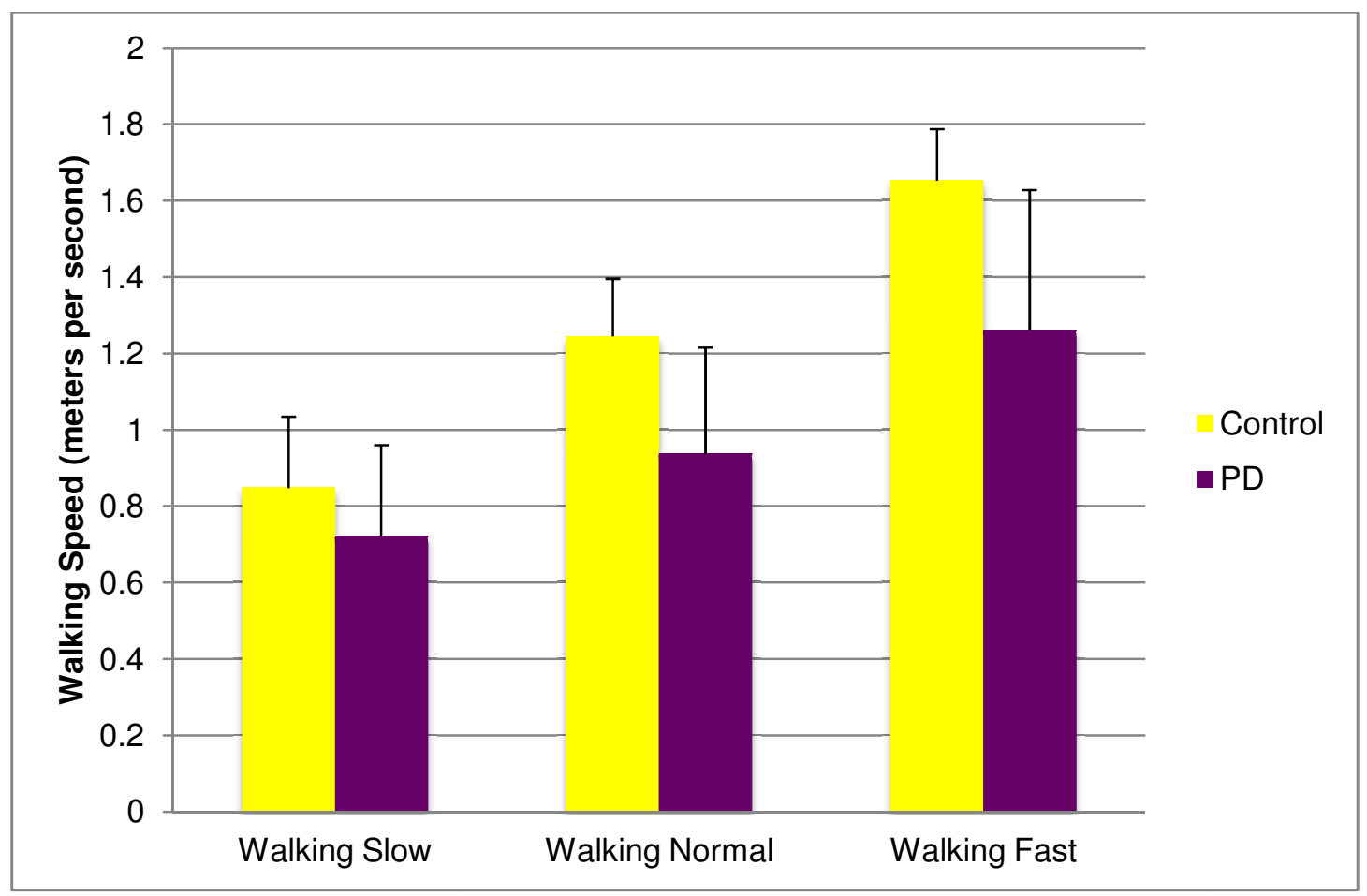

Figure 23. Average walking speed by walking condition at an interlocutor at an interlocutor distance of six meters. Standard deviations appear as error bars.

\subsubsection{Main Effects Related to Walking Speed: Interlocutor Distance}

The main effect of interlocutor distance was not significant $[F(1,23)=0.92, \mathrm{p}=.346]$ and is illustrated in Figure 24 with associated means and standard errors listed in Table 27. This non-significant result indicates that both participant groups have similar walking speeds at both interlocutor distances (one meter and six meters). 
Table 27. Average Walking Speed at an Interlocutor Distance of One and Six Meters

\begin{tabular}{cc}
\hline 1 meter & 6 meters \\
\hline 1.13 & 1.11 \\
$(0.04)$ & $(0.04)$ \\
\hline
\end{tabular}

*Walking speed values are in meters per second. Standard error scores appear in parenthesis below means.

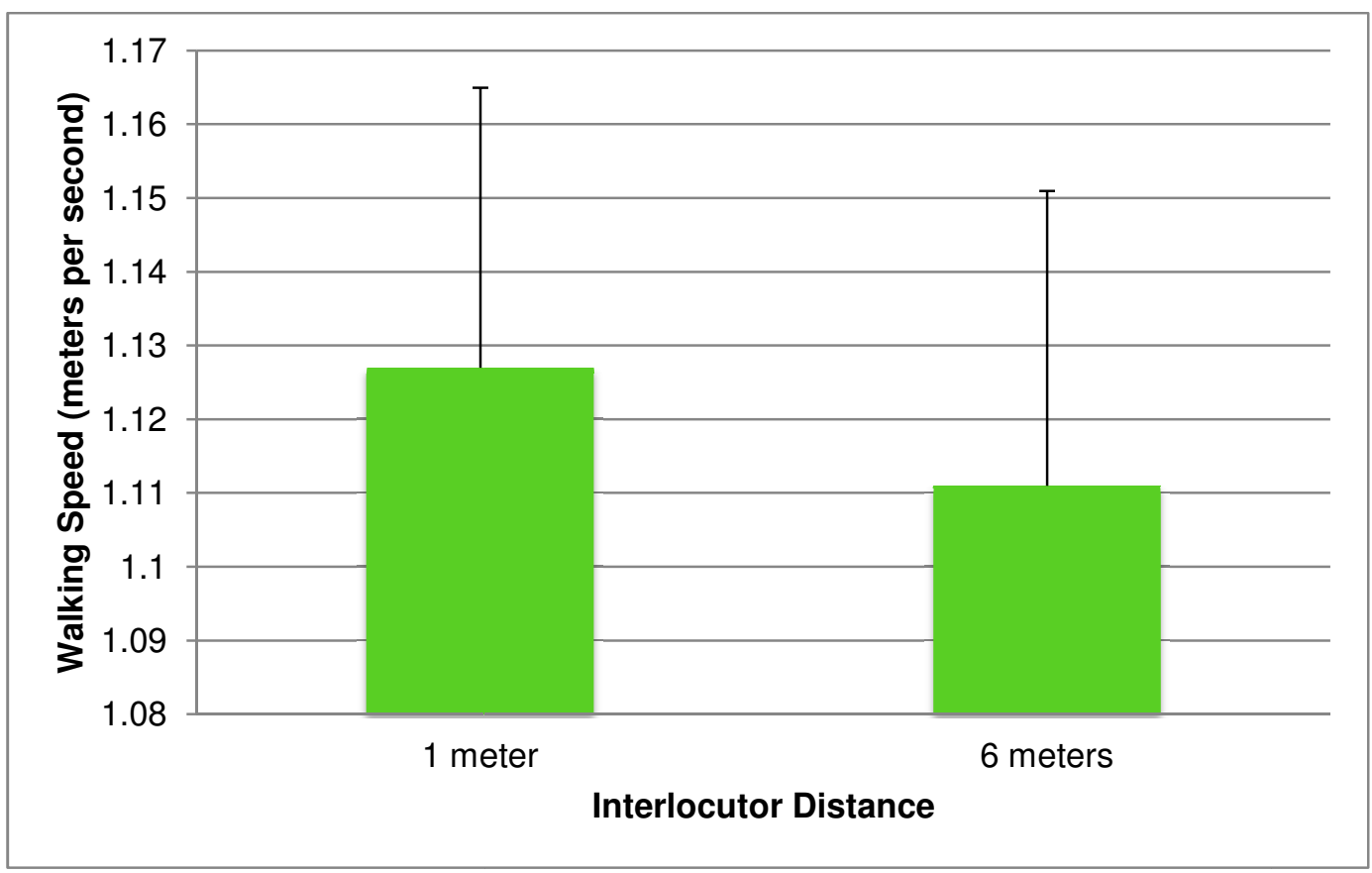

Figure 24. Average walking speed at an interlocutor distance of one and six meters. Standard error scores appear as error bars.

\subsubsection{Interactions Related to Walking Speed: Group, Interlocutor Distance, and}

\section{Walking Condition}

The result of the interlocutor distance by group interaction was not significant $[F(1,23)=0.28, \mathrm{p}=.602]$. This non-significant result indicates that an increase in 
interlocutor distance does not effect the speed of walking in both participant groups in a similar way.

The result of the interlocutor distance by walking condition interaction was not significant $[F(2,22)=0.02, \mathrm{p}=.972]$. This non-significant result indicates that the changes in walking speed that occurred across the walking speed conditions (slow to fast) were similar for the one meter and six meter interlocutor distances.

The three way interaction involving group, interlocutor distance, and walking condition was not significant $[F(2,22)=0.84, \mathrm{p}=.538]$.

\subsection{Stride Length (meters)}

The other secondary objective of this study was to examine the effect of concurrent talking on average stride length in individuals with Parkinson's disease and healthy age-equivalent controls. A three factor, repeated measures ANOVA was performed using participant group as the between groups factor with two levels (control and PD). The two within group factors included talking versus not talking and the type of walking task. The talking factor had two levels (talking and not-talking). The factor related to the type of walking task had three levels (walking at a speed perceived by the participant to be two times slower than their habitual walking speed, walking at a habitual walking speed, and walking at a speed perceived by the participant to be two times faster than their habitual walking speed). The results of the three-way ANOVA are presented in separate sections related to the main effects (group, talking versus not-talking, and walking condition) and the interactions. The results are summarized in Figures 25 and 26, with associated means and standard deviations listed in Tables 28 and 29. The detailed 
results of the three-way ANOVA related to stride length and concurrent talking are presented in Appendix K.

Table 28. Average Stride Length by Walking Condition while Concurrently Talking

\begin{tabular}{ccc}
\hline & Control & PD \\
\hline Walking Slow & $0.62(0.07)$ & $0.52(0.12)$ \\
Walking Normal & $0.71(0.06)$ & $0.58(0.13)$ \\
Walking Fast & $0.83(0.06)$ & $0.67(0.15)$ \\
\hline
\end{tabular}

*Note: Stride length values are in meters. Standard deviations appear in parenthesis beside means.

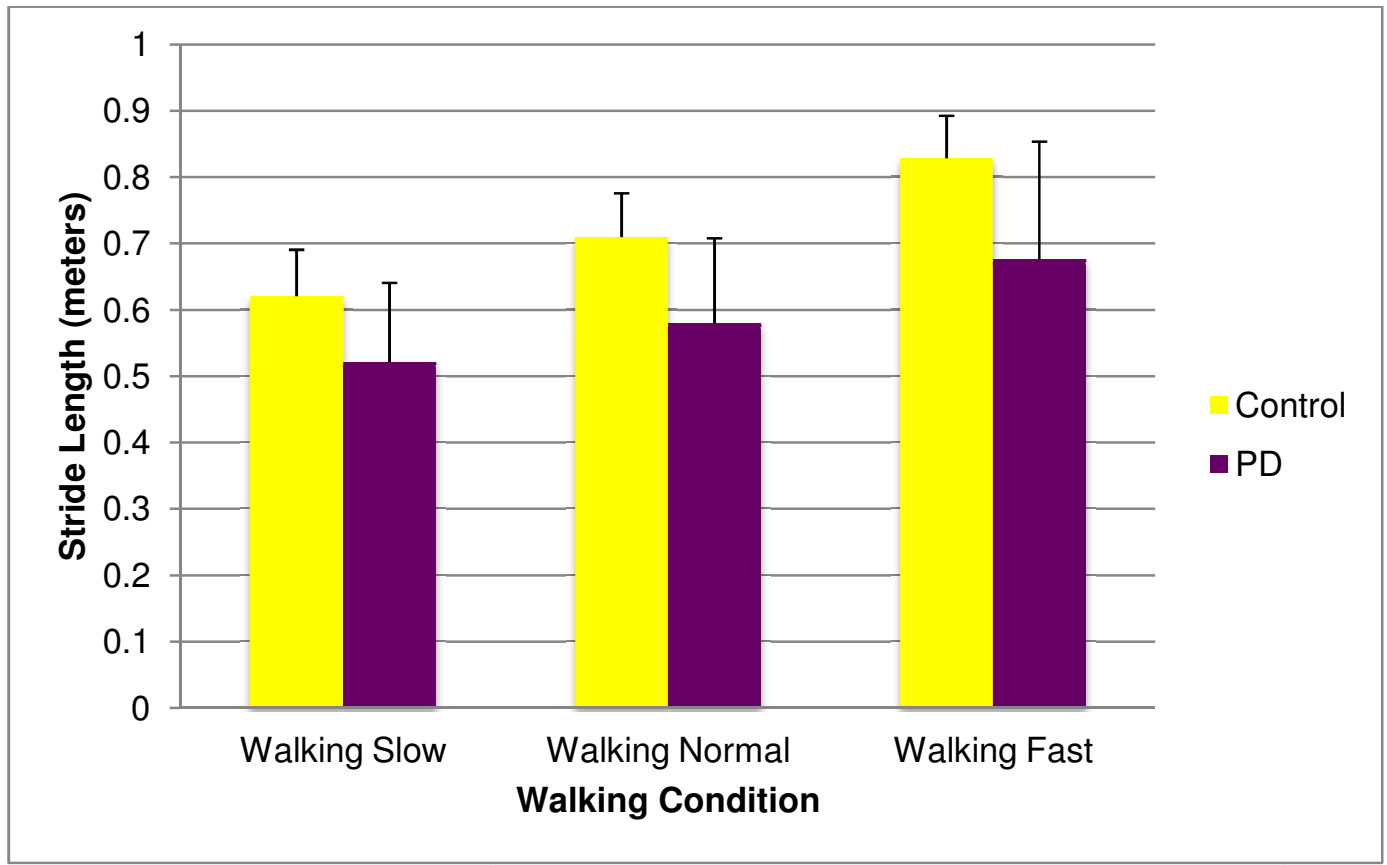

Figure 25. Average stride length by walking condition while concurrently talking. Standard deviations appear as error bars. 
Table 29. Average Stride Length by Walking Condition while not Talking

\begin{tabular}{ccc}
\hline & Control & PD \\
\hline Walking Slow & $0.63(0.08)$ & $0.57(0.11)$ \\
Walking Normal & $0.75(0.05)$ & $0.65(0.13)$ \\
Walking Fast & $0.85(0.07)$ & $0.74(0.11)$ \\
\hline
\end{tabular}

*Note: Stride length values are in meters. Standard deviations appear in parenthesis beside means.

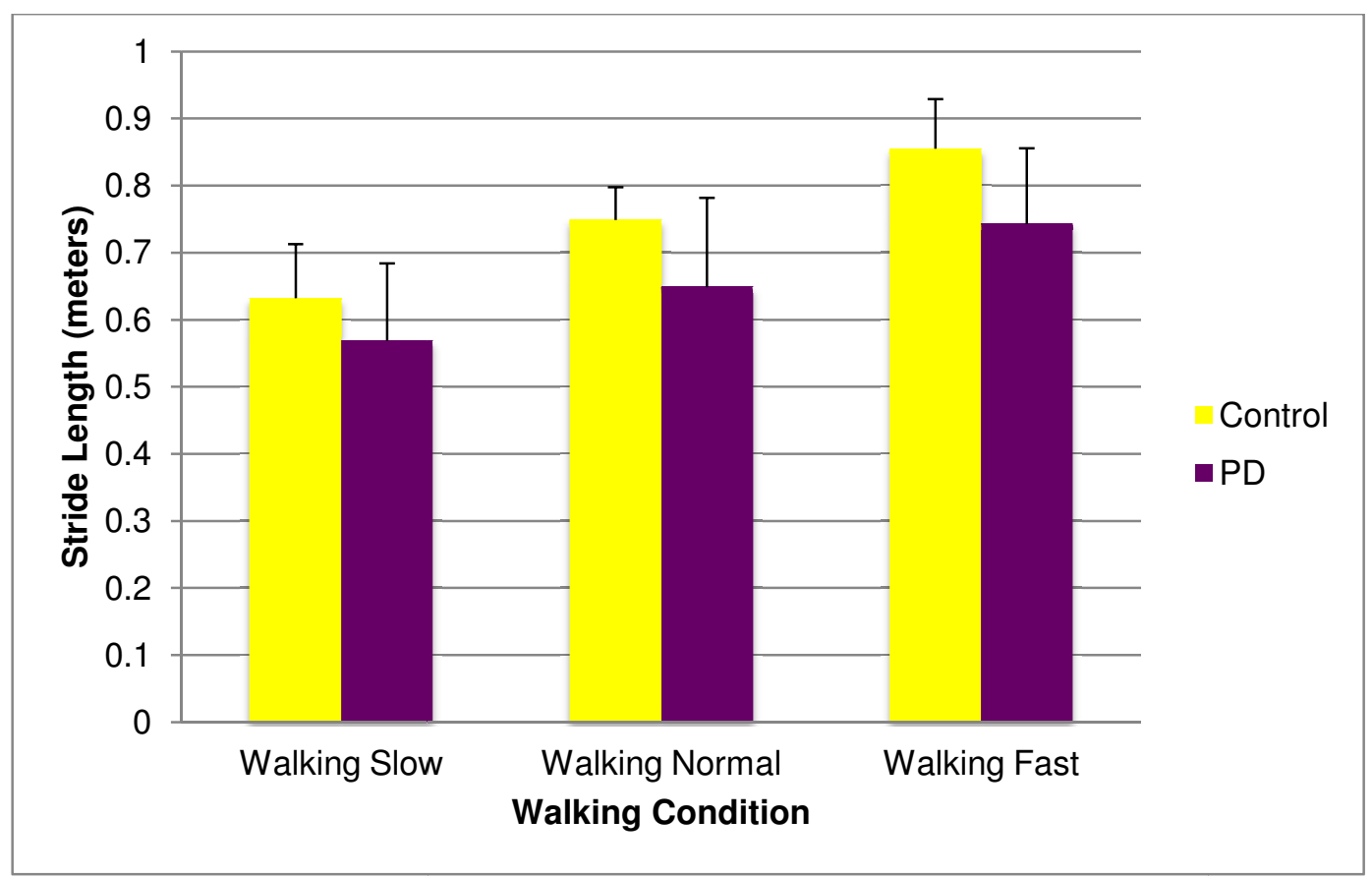

Figure 26. Average stride length by walking condition while not talking. Standard deviations appear as error bars.

\subsubsection{Main Effects Related to Stride Length: Group, Talking Condition, and Walking Condition}

The main effect of group was significant $[F(1,26)=15.18, \mathrm{p}=0.001]$ and is illustrated in Figure 29 with associated means and standard error scores in Table 32. This significant result indicates that there was an overall difference in stride length between 
participant groups across all talking versus not-talking and walking conditions. The PD participants had a consistently shorter stride length than the control participants.

Table 30. Average Stride Length for Control and PD Participants

\begin{tabular}{cc}
\hline PD & Control \\
\hline 0.62 & 0.73 \\
$(0.02)$ & $(0.02)$
\end{tabular}

*Note: Stride length values are in meters. Standard error scores appear in parenthesis beside means.

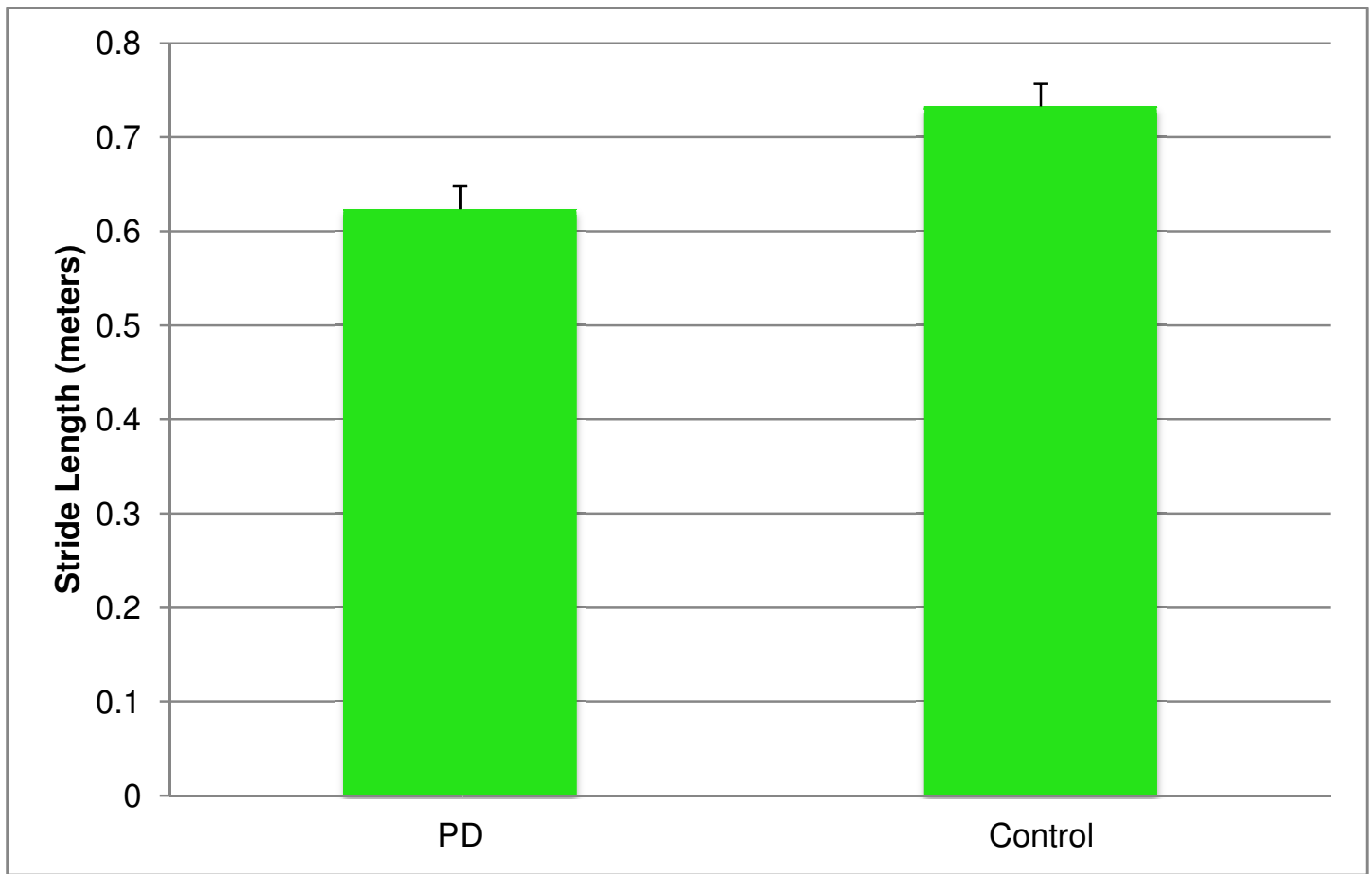

Figure 27. Average stride length for control and PD participants. Standard error scores appear as error bars.

The main effect of the talking condition (i.e. talking versus not talking) was also significant $[F(1,26)=39.82, \mathrm{p}=0.000]$ and is illustrated in Figure 30 with associated 
means and standard error scores in Table 33. This significant result indicates that stride length is significantly greater when walking only, than when concurrently talking and walking.

Table 31. Average Stride Length While Concurrently Talking and Walking, and Walking Only

\begin{tabular}{cc}
\hline Talking & Not Talking \\
\hline 0.66 & 0.7 \\
$(0.02)$ & $(0.02)$ \\
\hline
\end{tabular}

*Note: Stride length values are in meters. Standard deviations appear in parenthesis beside means..

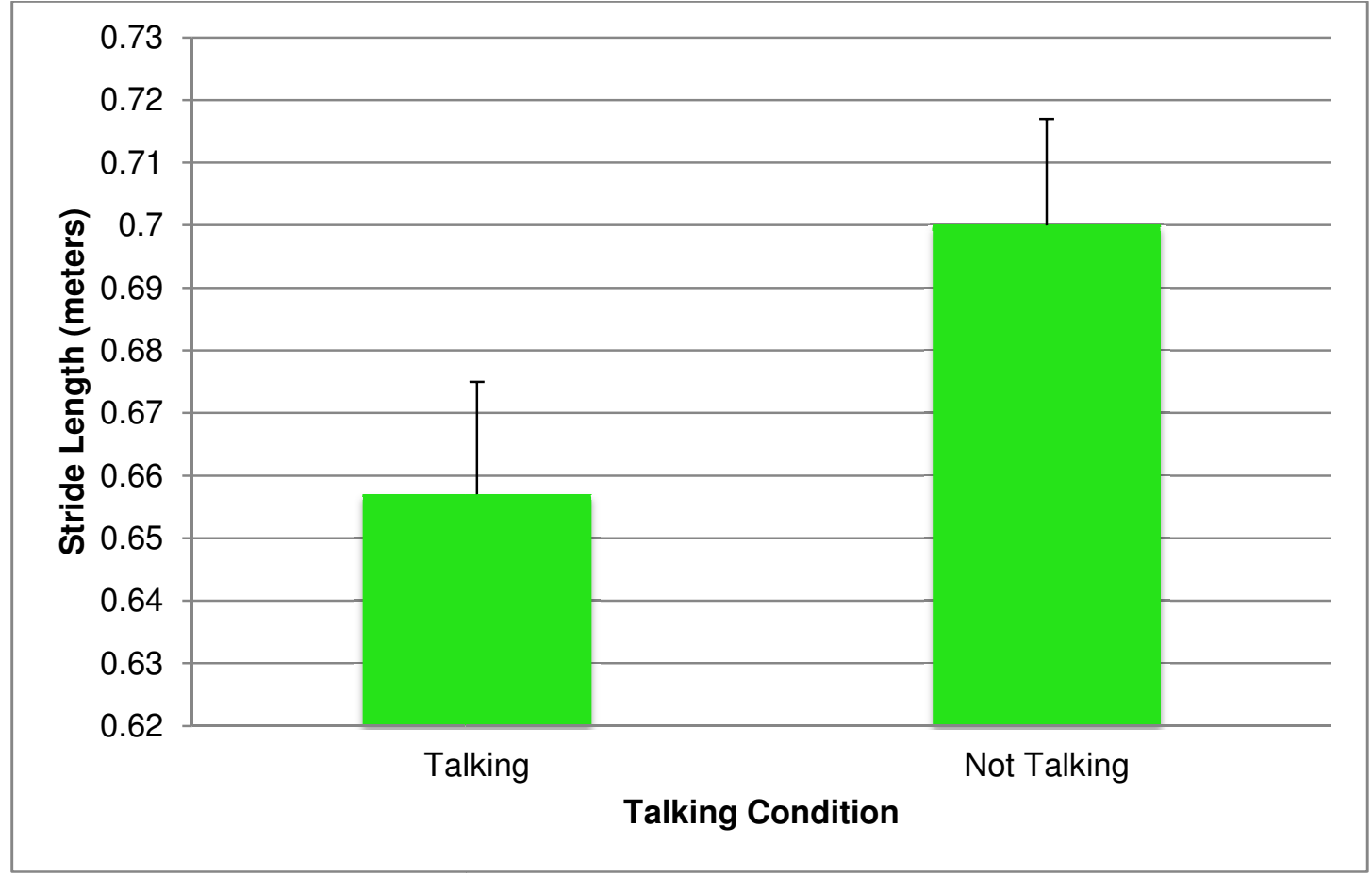

Figure 28. Average stride length while concurrently talking and walking, and walking only. Standard deviations appear as error bars. 
The main effect of walking condition was also significant

$[F(2,25)=88.58, \mathrm{p}=0.000]$ and is illustrated in Figure 31 with associated means and standard error scores listed in Table 34. This significant result indicates that both participant groups had significant differences in stride length across the three walking conditions. For example, both participant groups showed an increase in stride length as they shifted from the slow walking condition to the fast walking condition.

Table 32. Average Stride Length Across Walking Speed Conditions

\begin{tabular}{ccc}
\hline Slow & Normal & Fast \\
\hline 0.58 & 0.67 & 0.77 \\
$(0.02)$ & $(0.02)$ & $(0.02)$ \\
\hline
\end{tabular}

*Note: Stride length values are in meters. Standard deviations appear in parenthesis beside means.

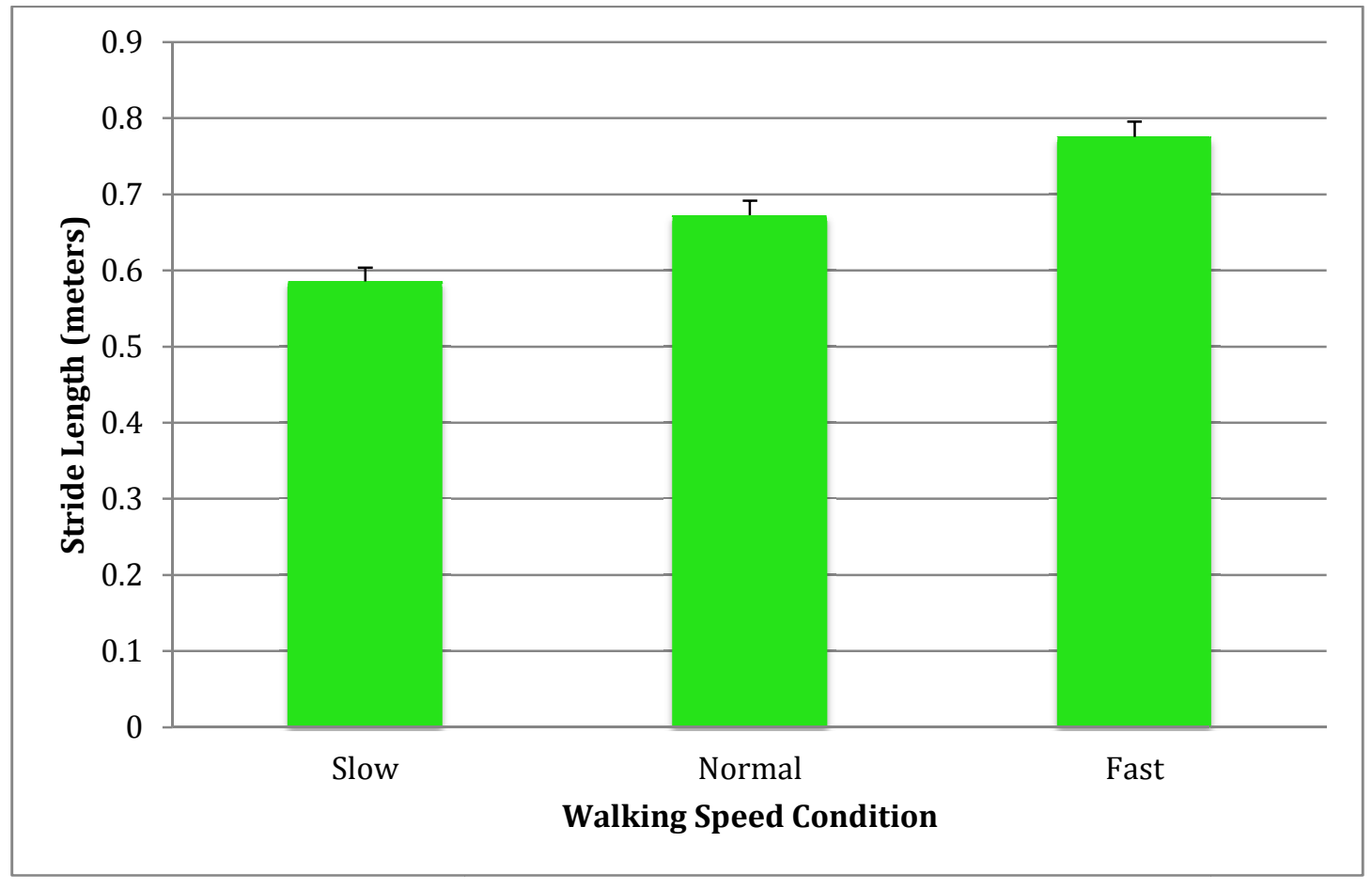

Figure 29. Average stride length across walking speed conditions. Standard deviations appear as error bars. 


\subsubsection{Interactions Related to Stride Length: Group, Talking Condition, and Walking Condition}

The result for the walking condition by group interaction was not significant $[F(2,25)=1.26, \mathrm{p}=0.118]$. This non-significant result indicates that the PD and controls groups had similar changes in stride length across all walking conditions.

The result for the talking condition by group interaction was significant $[F(1,26)$ $=6.58, \mathrm{p}=0.016]$. This significant result indicates that concurrent talking while walking had a different effect on stride length in the PD group than the control group. In particular, the PD group showed a relatively greater reduction in stride length than that of the controls when they shifted from the not talking to the talking condition.

The result for the walking condition by talking condition interaction was not significant $[F(2,25)=2.67, \mathrm{p}=0.131]$. This non-significant result indicates that talking and not talking had similar effects on stride length across all walking conditions.

The three way interaction involving group, talking versus not talking, and walking condition was not significant $[F(2,25)=2.67, \mathrm{p}=.088]$.

A second three factor, repeated measures ANOVA was performed to examine whether interlocutor distance had an effect on stride length in individuals with Parkinson's disease and healthy age-matched controls. The participant group was the between groups factor with two levels (control and PD). The two within group factors included interlocutor distance and the type of walking task. The interlocutor distance factor had two levels (1-meter and 6-meters). The factor related to the type of walking task had three levels (walking at a speed perceived by the participant to be two times 
slower than their habitual walking speed, walking at a habitual walking speed, and walking at a speed perceived by the participant to be two times faster than their habitual walking speed). The results of the three-way ANOVA are presented in separate sections related to the main effects of interlocutor distance and the interactions. The results are summarized in Figures 27 and 28, with associated means and standard deviations listed in Tables 30 and 31. The detailed results of the three-way ANOVA related to stride length and interlocutor distance are presented in Appendix L.

Table 33. Average Stride Length by Walking Condition at an Interlocutor Distance of One Meter

\begin{tabular}{ccc}
\hline & Control & PD \\
\hline Walking Slow & $0.62(0.07)$ & $0.52(0.13)$ \\
Walking Normal & $0.70(0.06)$ & $0.59(0.14)$ \\
Walking Fast & $0.83(0.06)$ & $0.68(0.16)$ \\
\hline
\end{tabular}

*Note: Stride length values are in meters. Standard deviations appear in parenthesis beside means. 


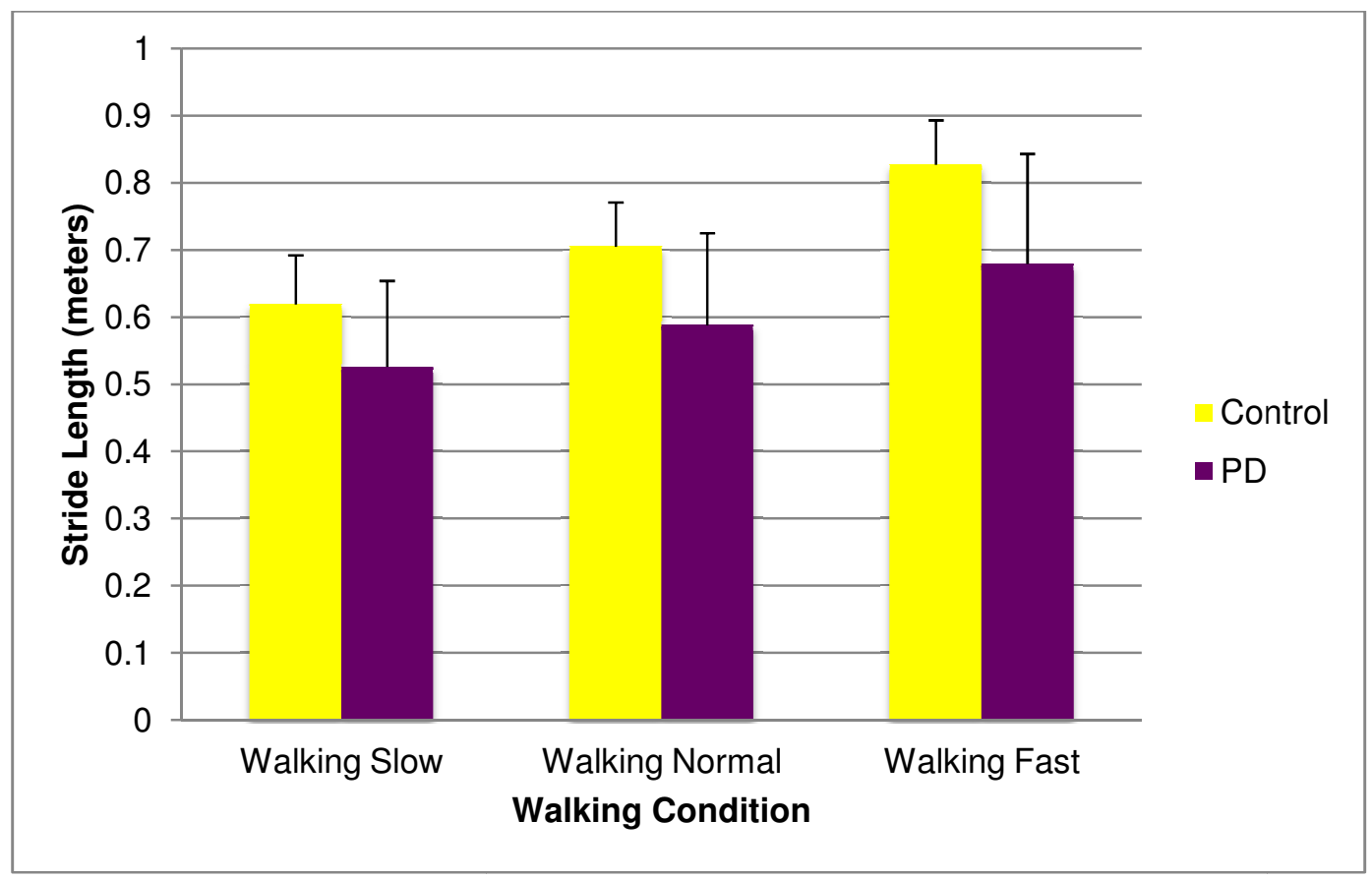

Figure 30. Average stride length by walking condition at an interlocutor distance of one meter. Standard deviations appear as error bars.

Table 34. Average Stride Length by Walking Condition at an Interlocutor Distance of Six Meters

\begin{tabular}{ccc}
\hline & Control & PD \\
\hline Walking Slow & $0.61(0.06)$ & $0.51(0.14)$ \\
Walking Normal & $0.71(0.05)$ & $0.59(0.14)$ \\
Walking Fast & $0.80(0.08)$ & $0.69(0.18)$ \\
\hline
\end{tabular}

*Note: Stride length values are in meters. Standard deviations appear in parenthesis beside means. 


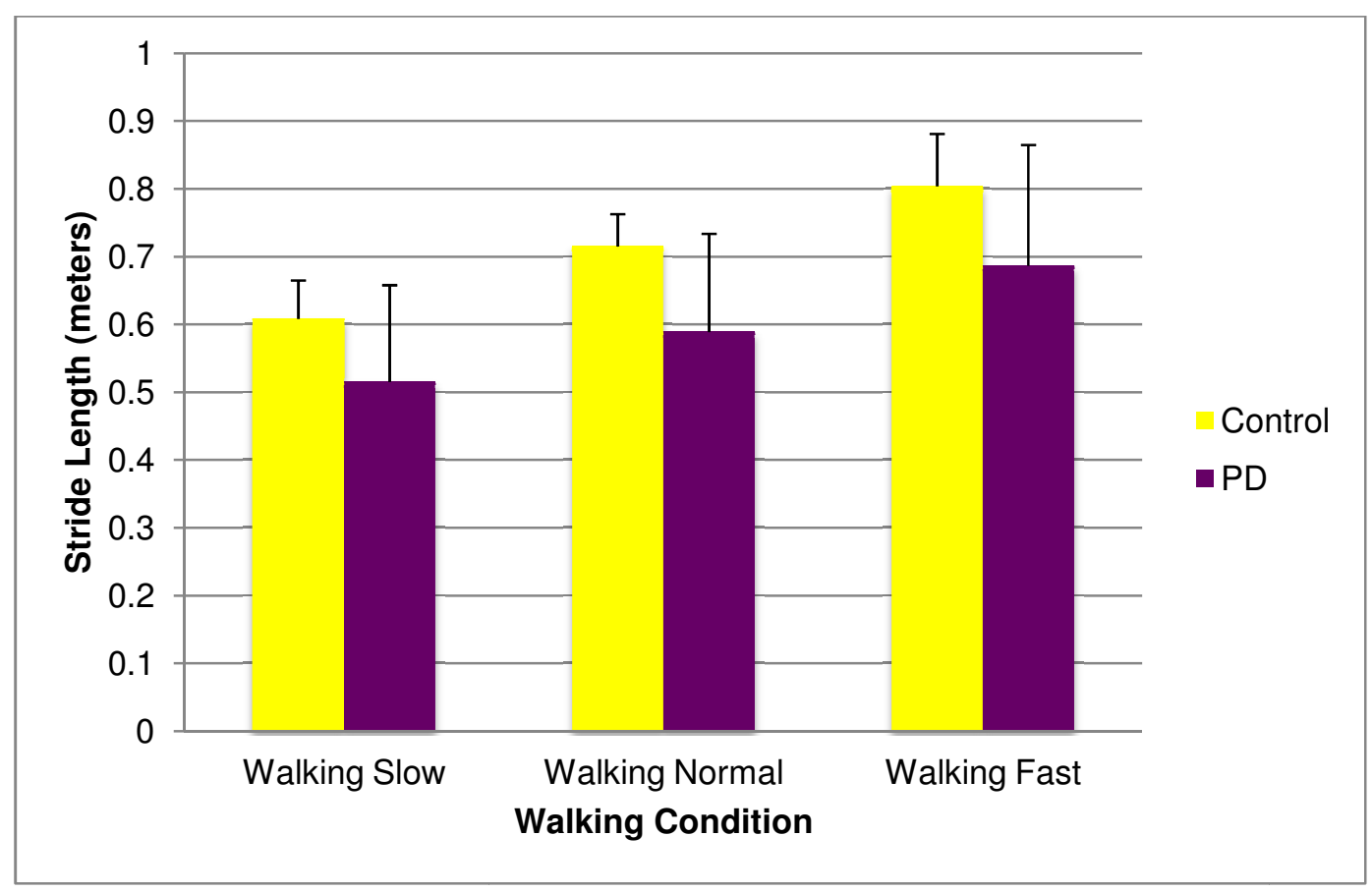

Figure 31. Average stride length by walking condition at an interlocutor distance of six meters. Standard deviations appear as error bars.

\subsection{Reliability}

To determine the inter-judge and intra-judge reliability of the conversational speech intensity and speech rate measures, and the walking speed and stride length measures, the original judge reanalyzed $10 \%$ of the data and $10 \%$ of the data was analyzed by a second judge. A bivariate correlation analysis revealed high intrajudge reliability for all measures, with correlation coefficients ranging from $.86-.99$. The analysis also revealed high inter-judge reliability for all measures, with correlation coefficients ranging from $.84-.99$. Table 35 summarizes the results of the correlation analysis used to obtain inter and intra-judge reliability estimates. This correlation analysis demonstrates good overall reliability between and within judges for conversational speech intensity, conversational speech rate, walking speed, and stride length measures. 
Table 35. Inter and Intra-Judge Reliability

Pearson Correlation Coefficients

\begin{tabular}{ccc}
\hline & \multicolumn{1}{c}{ Intra-Judge } & \multicolumn{1}{c}{ Inter-Judge } \\
\hline $\begin{array}{c}\text { Conversational Speech } \\
\text { Intensity (dB) }\end{array}$ & $.94, \mathrm{p}=.00$ & $.95, \mathrm{p}=.00$ \\
$\begin{array}{c}\text { Conversational Speech Rate } \\
(\text { WPM) }\end{array}$ & $.89, \mathrm{p}=.00$ & $.84, \mathrm{p}=.00$ \\
$\begin{array}{c}\text { Walking Speed }(\mathbf{m} / \mathbf{s}) \\
\text { Stride Length }(\mathbf{m})\end{array}$ & $.86, \mathrm{p}=.00$ & $.99, \mathrm{p}=.00$ \\
\hline
\end{tabular}




\section{Chapter 4}

\section{Discussion}

The goal of the present study was to examine the effect of concurrent walking tasks and interlocutor distance on conversational speech intensity and conversational speech rate in individuals with PD and controls. The main objectives of this study were to: 1) examine the effects of concurrent walking on conversational speech intensity and conversational speech rate; 2) examine the effects of interlocutor distance on conversational speech intensity and conversational speech rate; 3) examine the effects of concurrent talking on walking speed; 4) examine the effects of concurrent talking on stride length. The following sections will discuss the findings of the present study and relate these findings to previous research examining the effect of concurrent tasks on conversational speech intensity and speech rate, interlocutor distance, and concurrent task effects on walking performance. The limitations of the present study will also be discussed, along with clinical implications and recommendations for future research.

\subsection{Effect of Concurrent Walking Tasks and Interlocutor Distance on}

\section{Conversational Speech Intensity}

Conversational intensity of Parkinson's and control groups. The results of this study revealed a significant difference in conversational speech intensity values between the Parkinson and control groups. Individuals with PD produced a conversational speech intensity that was on average $4 \mathrm{~dB}$ lower than that of the control participants. In the current study, conversational speech intensity varied across walking conditions and 
interlocutor distance conditions, but the difference between PD and control participants remained consistently around 2-4 dB SPL. A 2-4 dB SPL change in intensity is equal to about a 40\% reduction in perceived loudness (Fox \& Ramig, 1997). This finding is in agreement with several previous studies that have reported that individuals with PD have conversational speech intensity levels that are 2-4 dB SPL lower than those of healthy age-equivalent controls (Fox \& Ramig, 1997; Ho et al., 1999; Adams et al., 2010).

Low speech intensity (hypophonia) is generally recognized as one of the distinctive speech symptoms of PD. It is estimated that 40-50\% of individuals with hypokinetic dysarthria will present with low speech intensity, and it is often the first speech symptom to become apparent in the early stages of the disease (Adams et al., 2010).

\section{Effect of interlocutor distance on conversational intensity in Parkinson's and}

control groups. The results of this study revealed a significant difference in conversational speech intensity values between the one-meter and six-meters interlocutor distances for both the PD and control participants. Both participant groups significantly increased their conversational speech intensity in response to the increase in interlocutor distance. The PD and control participants were on average $2.5 \mathrm{~dB}$ louder at an interlocutor distance of six-meters than one-meter. This is in agreement with Ho et al. (2002) and Adams et al. (2010) who found that both PD and control participants increased their speech volume to compensate for an increase in interlocutor distance. In addition, there was no significant interaction between groups at one-meter and sixmeters. This finding that individuals with PD demonstrate a normal pattern of intensity 
regulation relative to controls in response to increased interlocutor distance is consistent with previous studies (Ho et al., 1999; Adams et al., 2010).

\section{Effect of walking conditions on conversational speech intensity in}

Parkinson's and control groups. The results of this study revealed a significant difference in conversational speech intensity values between walking conditions for both the PD and control participants. Post hoc analyses revealed that individuals with PD and control participants had significantly greater conversational speech intensity during walking conditions relative to the sitting and standing conditions. In addition, walking fast was associated with the greatest increase in conversational speech intensity for both participant groups at one-meter interlocutor distance.

Several hypotheses were considered in relation to the effect of concurrent tasks on conversational speech intensity. The results of the present study appear to provide support for the energizing hypothesis. The energizing hypothesis proposes that there is an energizing effect on conversational speech intensity when speech is performed concurrently with other tasks. This energizing effect is proposed to cause speech intensity to increase during concurrent motor tasks such as walking, manual actions and other motor behaviours (Adams et al., 2010).

The findings of the present study are in agreement with the Adams et al. (2010) study, which found an increase in speech intensity in individuals with PD during a concurrent manual visuomotor tracking task. In addition, the results of this study are in agreement with the Dromey and Shim (2008) study, and the Dromey and Bates (2005) 
study that found an increase in speech intensity during concurrent manual tasks in young healthy participants.

In contrast to these findings, other studies have demonstrated a reduction in speech intensity while performing a secondary task. The control participants in the Adams et al. (2010) study demonstrated a reduction in speech intensity while performing a concurrent manual visuomotor tracking task. It was suggested that this failure to observe an energizing effect might have been related to the relatively easy nature of the concurrent manual task for the control participants. Adams et al. (2010) proposed that the energizing effect of concurrent tasks on speech intensity may be dependent on the difficulty level of the concurrent task. Interestingly, in the present study the walking condition by group interaction was not significant. This result indicates that both participant groups were affected by the walking tasks in a similar way. Both the PD and control groups had significantly greater conversational speech intensity while walking than while sitting or standing. In addition, both groups demonstrated a similar increase in conversational speech intensity across walking conditions. Both participant groups demonstrated a 2-5 dB increase in conversational speech intensity when they shifted from sitting to walking. This suggests that the concurrent walking task was sufficiently complex or challenging enough to produce an energizing effect on the conversational speech intensity of both the PD and control participants.

One additional study that failed to demonstrate an energizing effect of a concurrent task on speech intensity was the study by Ho et al. (2002). This study found that when individuals with PD produced a loud speech task involving loud counting of numbers there was a significant decrease in the speech intensity. This result suggests that 
the energizing effect of a concurrent task on speech intensity may not extend to conditions of high intensity speech production. Perhaps there is a ceiling effect that does not allow for increases in speech intensity when speech is already being produced at a high level of intensity.

Although the results of the present study appear to provide support for the energizing hypothesis, additional studies that involve the systematic examination of different speech tasks and the careful evaluation of various concurrent task parameters are required in future studies.

\section{Effect of walking speed on conversational speech intensity in Parkinson's}

and control groups. In general, the speed of walking was found to have an effect on conversational speech intensity. The post-hoc analyses revealed that both PD and control participants had significantly greater speech intensity while walking fast than while walking at a comfortable speed or walking slow at one meter interlocutor distance.

In the Dromey and Shim (2008) study and the Dromey and Bates (2005) study, the authors proposed that greater speech intensity may be the result of an overall increase in effort caused by the introduction of a concurrent task. The results of this study are consistent with this hypothesis, suggesting that an increase in effort (by walking faster) in one task translates to increased effort (greater speech intensity) in the other task.

This is the first study to provide evidence that increasing physical effort in a highly pre-programmed functional task, such as walking, increases conversational speech intensity in individuals with PD. This result suggests that physical effort as well as task complexity may play a role in the energizing effect of concurrent tasks on speech 
intensity. Further research should investigate the interaction between lower limb activities and motor speech acoustics, as well as the effect of increased physical effort of dual-task activities on motor speech acoustics.

\section{Effect of standing on conversational speed intensity in Parkinson's and}

control groups. The results of this study revealed no significant difference in conversational speech intensity between sitting and standing for both PD and control participants. However, both the PD and control participants demonstrated a slight reduction in conversational speech intensity ( $0.5 \mathrm{~dB}$ and $1.5 \mathrm{~dB}$ respectively) when standing, compared to sitting.

It was hypothesized that standing would enhance speech intensity by preactivating or exciting the motor cortex. This hypothesis was based on the Meinzer et al. (2011) study that reported an enhancing effect of standing on word retrieval performance in individuals with aphasia. The authors suggested that pre-activation of the motor cortex could be used to excite the language network. In the present study it was hypothesized that standing may have a similar excitatory effect on the speech production system. Interestingly, the results of the present study did not show an excitatory effect of standing on the speech production parameter of speech intensity. Future studies are required to determine if there are excitatory effects of standing on other parameters of speech production (i.e., speech fluency, voice quality).

Holmes et al. (2010) investigated concurrent interference on postural control and proposed that individuals with PD and healthy older adults employ a 'posture-first principle' under dual-task conditions. This principle suggests that people prioritize 
balance over concurrent tasks, and this prioritization becomes more pronounced with high complexity tasks, such as talking. It is possible that both participant groups attended more to balance and postural stability than to motor speech performance. However, if this prioritization of balance caused interference in motor speech performance, it would be expected that the reduction in speech intensity would be exacerbated while walking, especially in the fast walking condition. O'Shea et al. (2002) stated that fast walking speeds require greater balance control because of the rapidly changing accelerations of the center of mass and the reduction in double support time. Therefore, it is possible that an overall increase in physical effort, by walking, has a more powerful effect on motor speech performance than the posture-first strategy.

In general, the results of the present study indicate that concurrent walking tasks can have a significant effect on conversational speech intensity. These effects need to be given consideration in future attempts to develop a comprehensive model of speech intensity regulation and in future attempts to understand the problem of hypophonia in PD.

\subsection{Effect of Concurrent Walking Tasks and Interlocutor Distance on}

\section{Conversational Speech Rate}

Conversational speech rate of Parkinson's and control groups. The results of this study revealed no significant difference in conversational speech rate between the PD and control participants. The results of this study are in agreement with previous studies, which found no significant difference in the speaking rate between individuals with PD and healthy adults (Flint et al., 1992; Walsh \& Smith, 2012; Tjaden \& Wilding, 2011). 
Previous studies have shown that typical discourse speaking rates for healthy adults is between 143 and 183 WPM (words per minute) or 195 and 236 SPM (syllables per minute). In contrast, the mean conversational speech rate for the control participants in the present study was 223.5 WPM, which is slightly faster than previous reports.

A few previous studies have suggested that only a small proportion of individuals with PD (6-13\%) demonstrate an abnormally rapid speech rate (Adams \& Dykstra, 2009), however most previous studies have found that speech rate in PD is comparable to that of age-equivalent controls (Adams \& Dykstra, 2009).

It should be noted that one recent study by Tjaden and Wilding (2011) reported no significant difference between PD and control participants for speech rate but a significant difference for pause durations. PD participants had significantly longer pause durations than controls. This study by Tjaden and Wilding (2011) involved speech during a reading aloud task. Additional research is required to determine the characteristics of pause durations in PD speakers during conversational speech.

\section{Effect of interlocutor distance on conversational speech rate in Parkinson's}

and control groups. The results of this study revealed no significant difference in conversational speech rate at the one-meter and six-meters interlocutor distances for both the PD and control groups. This may suggest that the mechanisms involved in increasing speech volume do not effect speaking rate. This result was somewhat unexpected.

Previous studies have found that loud speech can be associated with a reduction in speech rate (Wenke, Theodoros, \& Cornwell, 2011; Schulman, 1989). Thus, it is somewhat surprising that the increased speech intensity associated with greater interlocutor distance 
did not produce a corresponding reduction in speech rate in the present study. It should be noted that there was a tendency for the control participants to use a slightly slower speech rate as they increased their interlocutor distance from one to six meters. In contrast to this, the PD participants showed a tendency to increase their rate of speech as the interlocutor distance increased. Additional studies are required to investigate the relationship between speech intensity and speech rate in both PD and control participants. These future studies should examine the effect of a wide range of changes in speech intensity on speech rate. The present study was associated with significant changes in speech intensity $(+2 \mathrm{~dB})$ however larger changes in speech intensity may be necessary in order to observe significant effects on speech rate.

\section{Effect of walking condition on conversational speech rate in Parkinson's and}

control groups. In general, the results of this study revealed no significant difference in conversational speech rate between the five walking conditions. In addition, there was no significant difference between groups across the five walking conditions. The present study is in agreement with the finding by Ho et al. (2002) that control participants had no significant change in speech rate while performing concurrent manual tracking task. The

present study is also in agreement with a study by Pohl, Kemper, Siengsukon, Boyd, and Vidoni (2011) that looked at the effect of walking on conversational speech rate in older adults with and without a stroke. For both of these participant groups, Pohl et al. (2011) found no significant difference in the conversational speech rate between the walking and not walking conditions. In contrast, Kemper, Herman, and Lian (2003) investigated conversational speech rate in healthy older adults and found a significant reduction in 
conversation speech rate while performing concurrent finger tapping or walking tasks.

The inconsistencies in speaking rate for both healthy control participants and individuals with PD may be partially explained by methodological differences across these concurrent speech studies. Both the Ho et al. (2002) and Kemper et al. (2003) studies used concurrent manual tasks. On the other hand, the Pohl et al. (2011) study used a concurrent walking task and they elicited conversational speech using a methodology that was very similar to that of the present study. The Pohl et al. (2011) and the present study failed to show an effect of concurrent walking on speech rate. Additional studies are required to compare the effects of concurrent walking tasks versus concurrent manual tasks on speech rate and other aspects of speech production.

\subsection{Effect of Concurrent Talking, Interlocutor Distance and Walking Condition on Walking Speed}

Walking speed of Parkinson's and control groups. The results of this study

revealed that the PD participants walked significantly slower than the control participants across all of the talking and not-talking walking conditions. This finding is in agreement with previous studies that suggest that gait impairments are a common consequence in $\mathrm{PD}$, and reduced gait velocity is one of the most distinctive gait impairments (Rochester et al., 2005; O’Shea et al., 2002; Morris et al., 1994b; Mak, 2013). In the present study, the mean gait velocity of the control participants was 1.27 meters per second, which is in agreement with a number of previous studies that suggest habitual walking speed is between 0.88 and 1.36 meters per second (Rochester et al., 2005; O'Shea et al., 2002; Morris et al., 1994b; Mak, 2013). In contrast, the PD participants in the present study 
walked at a mean gait velocity of 0.95 meters per second, which is slightly faster than previously reported for individuals with PD (0.56 - 0.83 meters per second [Rochester et al., 2005; O'Shea et al., 2002; Morris et al., 1994b; Mak, 2013]).

It is also interesting to note that both participant groups did not actually produce a two times faster or two times slower walking speed in the particular walking speed conditions. For example, when the participants were asked to walk two times faster than their normal walking speed $(1.2 \mathrm{~m} / \mathrm{s})$ they only increased their walking speed by about $33 \%(+0.4 \mathrm{~m} / \mathrm{s})$ instead producing a doubling or a $100 \%$ increase in their walking speed $(2 * 1.2=2.4 \mathrm{~m} / \mathrm{s})$. The psychophysical relationship between the perceived and actual magnitude of a stimulus has been described for many types of physical stimuli (Stevens, 1962; Grosjean \& Lane, 1973). In many previous psychophysical studies, the perceived magnitude of a self-generated activity or movement has been found to be exponentially (a power function) related to the actual magnitude of the physical stimulus (Stevens, 1962). In speech production, this psychophysical relationship is referred to as an autophonic function. Autophonic functions have been described for speech intensity/loudness and speech rate (Lane, Catania \& Stevens, 1961; Grosjean \& Lane, 1973). For speech rate, an autophonic power function of 2.6 has been determined (Grosjean \& Lane, 1973). This power function is the linear function that was obtained from a log-log plot of perceived and actual speech rate. This function (2.6) means that in order for a person to actually produce a rate of speech that is two times faster they would need to have the perception that they are producing a rate of speech that is six times faster. A function that is similar to this six-fold relationship appears to be present for the walking speed results that were obtained in the present study. When the participants were asked to double their walking 
speed they only increased their walking speed by about $33 \%$. Thus, it appears that the participants would need to be asked to try to walk at approximately six times their normal walking speed in order for them to produce a walking speed that was actually two times faster than their normal speed. This perceived-to-actual psychophysical function for walking speed needs to be further investigated and defined in future studies.

\section{Effect of interlocutor distance on walking speed in Parkinson's and control}

groups. The results of this study revealed no significant difference in walking speed at one-meter and six-meters interlocutor distance. This result suggests that the mechanism involved in increasing vocal output appears to have little or no effect on walking speed. Additional concurrent walking and talking studies that involve greater increases in speech intensity $(+2 \mathrm{~dB})$ are required to examine this proposed null relationship in greater detail.

\section{Effect of changing walking speed in Parkinson's and control groups. The} results of this study revealed that walking speed was significantly different across the three walking speed conditions. Both participant groups walked significantly slower in the slow walking condition, and significantly faster in the fast walking condition.

In the fast walking condition, the PD participants were walking at a speed relative to the control participant's normal walking speed. In the walking only condition the fast walking speed for the PD participants was 1.24 meters per second, and the normal walking speed of the control participants was 1.26 meters per second. The same relationship was found while concurrently talking. The fast walking speed for the PD participants was 1.46 meters per second, and normal walking speed of the control 
participants was 1.41 meters per second. A study by Morris et al. (1994b) had similar findings, and suggested that individuals with PD have the capacity to walk at the normal speed for age-equivalent controls, but walking speed is habitually reduced because stride length is shortened. When individuals with PD are asked to walk at faster speeds, their stride length approximates the stride lengths of the healthy age-equivalent controls walking at a normal speed. In addition, the previous studies investigating walking speed have incorporated stride length cues, or have used pre-determined walking speeds on a treadmill to demonstrate that PD participants have the capacity to walk at the normal speed of healthy age-equivalent participants. This study reveals that when simply asked to walk faster, without the additional assistance of stride length cues, the PD participants selected a fast walking speed that approximates the normal walking speed of healthy ageequivalent controls. This finding was replicated during the concurrent talking condition.

The interaction between the walking speed conditions and the participant group was significant. This result reveals that the PD group and the control group regulated walking speed differently across walking speed conditions. A potential explanation this significant interaction may be because the PD group had a more limited range in walking speed than the control group. From the slow to fast walking speed conditions, the control group had a range from 0.85 meters per second to 1.84 meters per second, whereas the PD participants had a range from 0.86 meters per second to 1.46 meters per second. It appears that although the PD participants can walk at speeds relative to the habitual speed of the control group, they may not have the capacity to walk at the fast speed of the control group. A potentially useful method of evaluating the capacity of the PD participants would be to ask them to walk at their maximum speed in future studies. This 
task was given consideration in the planning of the present study but the risk of falls in the PD participants was a major concern. Future studies involving maximum walking speed in PD participants may need to consider the use of a support harness or other device to minimize the risk of falls.

The more restricted range of walking speeds of the PD participants also suggests that the psychophysical relationship related to the perceptual scaling of walking speed may be abnormal in the PD participants. Additional studies, involving the systematic perceptual scaling of a wide range of walking speeds, are required to investigate the psychophysical relationship related to the perception of walking speed in PD.

Of potential importance for future studies involving the perception of walking in PD, were the frequent verbal reports by the PD participants who found that the slow walking condition was the most difficult walking condition in the present study. It may be important to examine the perception of walking difficulty or the perception of walking effort in future studies of walking speed in PD.

Effect of concurrent talking on walking speed. The results of this study revealed that both participant groups walked significantly slower while talking, than while walking only. This is in agreement with a number of studies that have found that talking significantly effects walking performance (Morris et al., 1996; O’Shea et al., 2002; Bloem et al., 2004; Rochester et al., 2008; Kelly et al., 2012). A substantial amount of research has been dedicated to investigating the effects of concurrent tasks on walking performance because individuals with PD are considered to be at an increased risk for falls during concurrent activities (Bloem et al., 2004; Morris et al., 1996). The results of 
these studies provide consistent evidence that talking (or performing other types of concurrent tasks) significantly deteriorates walking performance (Galletly \& Brauer, 2005; O'Shea et al., 2002; Rochester et al., 2004; Morris et al., 1996; Bloem et al., 2004). Of additional interest was the finding of a relatively similar (negative) effect of talking on the walking speed of the PD and control participants. This was supported by the failure to find a significant group by talking condition interaction for the measure of walking speed. However, the results demonstrate that the PD participants experienced a greater reduction in walking speed than the control participants, although the interaction did not reach statistical significance. The control participants experienced an $11 \%$ reduction in walking speed while concurrently talking compared to walking only, while the PD participants experienced an $18 \%$ reduction in walking speed. A similar result was obtained in a previous study by O'Shea et al. (2002), which found that both PD and control participants experienced a greater reduction in walking speed when they were engaged in concurrent talking and walking (O'Shea, et al. 2002). This is in agreement with a number of studies that suggest that individuals with basal ganglia dysfunction have greater difficulty coping with concurrent tasks, resulting in a greater reduction of walking speed and stride length relative to control participants (Rochester et al., 2008; O'Shea et al., 2002; Kelly et al., 2012).

\subsection{Effect of Concurrent Talking, Interlocutor Distance and Walking Condition on Stride Length}

Stride length of Parkinson's and control groups. The results of this study revealed that individuals with PD had a significantly shorter stride length than the control 
participants across all concurrent talking conditions and walking speed conditions. This result is consistent with a number of studies suggesting that walking performance is impaired in individuals with PD, and one of the fundamental problems in gait hypokinesia in PD is shortened stride length (Morris et al., 1996). The mean stride length for PD and control participants in the present study was 0.65 and 0.75 meters, respectively. Previous studies suggest that mean stride length values are between 1.24 and 1.47 meters for healthy controls, and 0.75 and 0.92 meters for PD participants. Interestingly, both participant groups in the present study had stride lengths that were shorter than 'typical' values previously reported in the literature.

Effect of concurrent talking on stride length. The results of this study revealed that both participant groups had a significantly shorter stride length while concurrently talking and walking. This is consistent with a number of studies investigating concurrent task effects on walking performance (see Kelly et al. (2012) for review), that found that stride length is significantly shorter while engaged in concurrent tasks for both PD and healthy age-equivalent controls. In the present study, the control participants had a stride length of 0.75 meters while walking and not talking at their normal comfortable speed. When engaged in a concurrent task, their stride length reduced by approximately $5 \%$ to 0.71 meters. In the normal walking and not talking condition, the PD participants had a stride length of 0.65 meters, which reduced by approximately $11 \%$ to 0.58 meters while concurrently talking. A similar pattern of results was found for the fast and slow walking condition. Thus, there was a significant reduction in walking stride length when the participants were talking and walking relative to when they were walking and not talking. 
Thus, the PD participants demonstrated a relatively greater reduction in stride length than the control participants when they shifted from the walking and not talking conditions to the walking and talking conditions. This was confirmed by the significant interaction for group by talking condition for stride length. This is in agreement with a number of previous studies that have demonstrated greater reductions in stride length in PD participants (approximately 14\%) than control participants (approximately 4\%) while engaged in concurrent task conditions (O'Shea et al., 2002; Rochester et al., 2008; Kelly et al., 2012).

Effect of changing walking speed on stride length. The results of this study revealed that walking speed significantly impacted stride length. Both the PD and control participants experienced longer stride lengths while walking fast, and the shortest while walking slow.

Both participants demonstrated a systematic increase in stride length as the walking speed increased. In the walking only condition the stride length of the control participants went from 0.63 meters to 0.75 meters to 0.85 meters (slow, normal, and fast walking conditions respectively). A similar relationship was found in the PD group, as their stride length went from 0.57 meters to 0.65 meters to 0.74 meters (slow, normal, and fast walking conditions respectively). At baseline, the PD participants have a smaller stride length, and this overall reduction remains across all walking speed conditions. In agreement with these findings, Morris et al. (1996) found that as walking speed increases, step size increases for both PD and control participants. 
In a previous study by Morris et al. (1994a b), they found that when gait velocity is controlled, the walking cadence is higher and stride length is shorter in individuals with PD than in healthy age-matched controls. 


\section{Chapter 5}

\subsection{Strengths and Limitations}

Strengths. Previous dual-task studies investigating speech parameters in PD have employed simple upper-limb motor tasks (finger-tapping, motor tracking), but it is difficult to generalize these findings to other types of behaviors or motor tasks. The present study investigated conversational speech intensity and rate in a more ecologically valid context than has been previously studied (talking while walking), and is the first to examine the effect of lower-limb concurrent tasks on these speech acoustic measures.

Limitations. The first limitation of the present study involves the unequal number of male and female PD participants. Although there were an equal number of male and female control participants ( 7 male, 7 female), the PD group had 2 female and 13 male participants. The gender differences may have influenced the conversational speech intensity results, as gender differences have been previously reported in studies investigating the effect of interlocutor distance on speech intensity. For example, Healey, Jones, and Berky (1997) found that during a reading aloud speech task, young women demonstrated greater increase in speech intensity in response to changes in interlocutor distance than young men. However, it should be noted that the young women in the Healey et al. (1997) study had an atypically low average speech intensity of $62 \mathrm{~dB}$ (calibrated at $15 \mathrm{~cm}$ ) at an interlocutor distance of 3 feet. On the other hand, the young men had a more typical average speech intensity $(74 \mathrm{~dB})$ that was $12 \mathrm{~dB}$ higher than that of the young women. Additional studies are required to replicate the Healey et al. (1997) 
study and to determine if there is a gender difference for the effect of interlocutor distance on conversational speech intensity in both young and older adults.

Another possible methodological limitation of the present study was that all of the walking conditions were performed during one 30-60 minute session. Participants moved through the walking conditions in a randomized order, which occasionally required 5-10 minutes of continuous walking at various walking speeds. Although all participants were encouraged to take occasional rests between walking speed conditions, participants may have become tired near the end of the experimental protocol. Fatigue may have influenced walking speed and stride length values in conditions at the end of the experimental protocol.

It should also be noted that the gait measurement methodology was not as refined or detailed as that of other gait-focused studies of PD. Many of these previous gait studies have used highly specialized kinematic and kinetic instrumentation. The present study used a fairly simple video methodology to obtain some potentially important findings related to the effect of conversational speech on gait but additional instrumental studies are required to confirm these findings and to provide a more comprehensive description of these effects on gait performance in PD.

A final potential limitation relates to the ecological validity of the conversational speech and walking tasks. The intention of the present study was to examine the concurrent effects of walking and talking in PD in a fairly natural or ecologically valid context. With this in mind, a conversational speech task was selected to represent a fairly typical speech activity and walking in a common gymnasium was selected to represent a fairly typical walking context. However, it is possible to imagine a walking and talking 
study with potentially greater ecological validity. For example, the speech task could involve typical conversations with a person who regularly talks with the participant (i.e. spouse). Likewise, the walking context could involve locations where the participant would typically go for walks (i.e. neighborhood street, local walking path, or shopping mall). Unfortunately, some of these contexts with potentially greater ecological validity are associated with significant methodological challenges. These include difficulty controlling the level of background noise during the speech recordings and the limited availability of gait measurement systems that can be used in a variety of naturalistic environments. Additional instrumental development is required to solve many of these methodological challenges.

\subsection{Future Directions}

The current study provides a novel perspective with regard to how concurrent tasks can effect conversational speech intensity in individuals with PD. Previous studies have shown support for an energizing effect on speech intensity while performing concurrent upper-limb activities, such as finger-tapping or visuomotor manual tasks (Dromey \& Bates, 2005; Dromey \& Shim, 2008; Adams et al., 2010). The present study demonstrated an energizing effect of lower-limb activities on conversational speech intensity in PD and control participants. Future research involving the careful evaluation of various concurrent task parameters should be performed to further investigate and define the energizing effect of secondary tasks on speech intensity and other speech task parameters. In addition, the finding that faster walking speeds can produce significantly greater conversational speech intensity needs further investigation. Future studies should 
examine the effect of increased physical effort during dual-task activities (upper and lower-limb) on speech intensity and other aspects of speech performance.

In addition, the results of the present study demonstrated that both participant groups did not actually produce a two times faster or slower walking speed in the targeted walking speed conditions. A psychophysical relationship between the perceived and actual magnitude of an activity or movement has been previously reported in studies investigating speech intensity and rate (referred to as autophonic function). The present study reveals that this psychophysical relationship appears to exist across many modalities. Additional studies, involving the systematic perceptual scaling of a wide range of walking speeds, are required to investigate the psychophysical relationship related to the perception of walking speed in PD.

Future studies should investigate the relationship between speech intensity and speech rate in both PD and control participants. Although the present study found no significant difference in speech rate between participant groups, a wider range of changes in speech intensity (greater than $2 \mathrm{~dB}$ ) may be necessary to observe significant effects on speech rate.

\subsection{Clinical Implications}

The effect of concurrent walking on conversational speech intensity needs to be given consideration in future attempts to develop a comprehensive model of speech intensity regulation and in future attempts to understand the problem of hypophonia in PD. In addition, the finding that fast walking causes a greater increase in conversational

speech intensity than walking at a comfortable speed may have important implications for 
speech therapy treatments that incorporate physical activities. For example, intensive phonatory-respiratory voice treatments for individuals with PD, such as the Lee Silverman Voice Treatment (Ramig, Sapir, Fox, \& Countryman, 2001) should consider incorporating activities that are physically challenging in a supervised environment to promote increased speech intensity.

In addition, Speech and Language Pathologists should consider assessing and treating individuals with PD in their natural communication environments since the variables used in the present study were chosen to mimic these natural contexts and were found to have significant effects on speech intensity. These natural communicative environments could include at home assessments, walking through a building, walking outside, or other locations that the patient frequently attends.

\subsection{Summary and Conclusions}

The primary objectives of the present study were to examine the effect of concurrent walking and interlocutor distance on conversational speech intensity and speech rate in 15 individuals with Parkinson's disease and 14 age-equivalent controls. The walking conditions included (1) sitting, (2) standing, (3) walking at a comfortable speed, (4) walking at a speed perceived by the participant to be two times slower than their comfortable walking speed, and (5) walking at a speed perceived by the participant to be two times faster than their comfortable walking speed. The walking conditions were performed over two interlocutor distances: 1-meter and 6-meters.

The conversational speech intensity results demonstrated that the average intensity of the PD participants across all walking and talking conditions was 
approximately $4 \mathrm{~dB}$ lower than the control participants. This result provides confirmation of hypophonia in the PD participants. All of the concurrent walking speed conditions were associated with increased speech intensity relative to the sitting condition. The fastest walking speed condition was associated with the greatest increase in conversational speech intensity for both the participant groups. Future research should attempt to define and further examine the energizing effect of concurrent walking, and the further energizing effect of activities of increased physical effort on conversational speech intensity in individuals with PD and healthy adults.

The present study found no significant difference in conversational speech rate between groups and across all walking and talking conditions. Future research should consider investigating speech rate in individuals with PD using additional measures such as the frequency and duration of conversational pauses or the variability and acceleration of intra-utterance speech rate.

The secondary objective of the present study was to examine the effect of concurrent talking on walking speed and stride length in individuals with PD and healthy age-equivalent controls. The results demonstrate that individuals with PD have a reduced gait velocity and shorter stride length relative to controls across all walking speed and talking conditions. In addition, concurrent walking and talking was associated with reduced walking speed and stride length values across all of the walking speed conditions, relative to walking only, in both participant groups. An interesting finding was that both participant groups did not actually produce a two times faster or two times slower waling speed in the particular walking speed conditions. For example, PD participants scaled walking speed by approximately $33 \%$ in the fast walking condition, 
instead of 100\%. Future research should investigate the actual-to-perceived scaling of walking speed in individuals with PD and healthy adults.

In general, the results of this study provide important new information about the effect of concurrent walking on speech motor performance in PD. In addition, this study provides support for an energizing effect of concurrent walking conditions on conversational speech intensity. This energizing effect may be an important consideration in the future development of assessment and treatment procedures for individuals with low speech intensity in PD. 


\section{References}

Adams, S.G., \& Dykstra, A. (2009). Hypokinetic Dysarthria. In M.R. McNeil (2Ed.). Clinical management of sensorimotor speech disorders (pp.166-186). New York: Thieme Medical Publishers.

Adams, S.G., Winnell, J., Jog, M. (2010). Effects of interlocutor distance, multi-talker background noise, and a concurrent manual task on speech intensity in Parkinson's disease. Journal of Medical Speech-Language Pathology, 18(4): 1-8.

Beradelli, A., Accornero, N., Argenta, M., Meco, G., Manfredi, M. (1986a) Fast complex arm movements in Parkinson's disease. Journal of Neurology, Neurosurgery, and Psychiatry, 49, 1146-9

Bernheimer, H., Birkmayer, W., Hornykiewicz, O., Jellinger, K., \& Seitelberger, F. (1973). Brain dopamine and the syndromes of Parkinson and Huntington. Clinical, morphological and neurochemical correlations. Journal of the Neurological Sciences, 20(4), 415-455.

Blanchet, P.G. (2002). Factors influencing the efficacy of delayed auditory feedback in treating dysarthria associated with Parkinson's disease [dissertation]. Louisiana: Louisiana State University and Agricultural and Mechanical College.

Bloem, B.R., Hausdorff, J.M., Visser, J.E., Giladi, N. (2004). Falls and freezing of gait in Parkinson's disease : a review of two interconnected, episodic phenomena. Movement Disorders, 19(8), 871-84

Boersma, P., Weenink, D. (2011). Praat : Doing phonetics by computer [Computer program]. Version 5.2.26, retrieved September 2012 from http://www.praat.org/

Braak, H., Rub, U., Gai, W.P., Del Tredici, K. (2003). Idiopathic Parkinson's disease: possible routes by which vulnerable neuronal types may be subject to 
neuroinvasion by an unknown pathogen. Journal of Neural Transmission, 110, 51736

Chiu, SL., Chou, LS. (2012). Effect of walking speed on inter-joint coordination differs between young and elderly adults. Journal of Biomechanics, 45, 275-280

Cooperman, L.F., Forwell, S.J., \& Hugos, L. (2002). Neurodegenerative Diseases. In Occupational Therapy for Physical Dysfunction (C.A. Tromblay \& M.V. Radomski ed., pp. 885-908). Baltimore, MD: Lippincott Williams \& Wilkins.

Darley, F.L., Aronson, A.E., \& Brown, J.R. (1969a). Differential diagnostic patterns of dysarthria. Journal of Speech and Hearing Research, 12, 264-269.

Darley, F.L., Aronson, A.E., \& Brown, J.R. (1975). Motor Speech Disorders (pp. 2-297), Philadelphia: W.B. Saunders.

Dirette, D.K. (2000). Progressive Neurological Disorders. In Conditions in Occupational Therapy: Effect on Occupational Performance (R.A. Hansen \& B. Atchison ed., pp.218-229). Baltimore, MD: Lippincott Williams and Wilkins.

Dromey, C., \& Bates, E. (2005). Speech interactions with linguistic, cognitive, and visuomotor tasks. Journal of Speech, Language, and Hearing Research, 48, 295 305

Dromey, C., \& Shim, E. (2008). The effects of divided attention on speech motor, verbal fluency, and manual task performance. Journal of Speech, Language, and Hearing Research, 51, 1171-1182

Duchin, S.W., \& Mysak, E.D. (1987). Disfluency and rate characteristics of young adult, middle-aged, and older males. Journal of Communication Disorders, 20, 245-257 
Duffy, J.R. (2005). Motor Speech Disorders, 2nd ed. Toronto, ON: Mosby.

Fahn, S. (2003). Description of Parkinson's disease as a clinical syndrome. Annals of the New York Academy of Sciences, 991, 1-14

Flint, A.J., Black, S.E., Campbell-Taylor, I., Gailey, G.F., Levington, C. (1992). Acoustic analysis in the differentiation of Parkinson's disease and major depression. Journal of Psycholinguistic Research, 21(5), 383-399

Fox, C., \& Ramig, L. (1997). Vocal sound pressure and self-perception of speech and voice in men and women with idiopathic Parkinson disease. American Journal of Speech-Language Pathology, 6, 85-93

Galletly, R., \& Brauer, S.G. (2005). Does the type of concurrent task affect preferred and cued gait in people with Parkinson's disease? Australian Journal of Physiotherapy, 51(3): $175-180$

Gentilucci, M. (2003). Grasp observation influences speech production. European Journal of Neuroscience, 17, 179-184.

Gentilucci, M., Benuzzi, F., Gangitano, M., \& Grimaldi, S. (2001). Grasp with hand and mouth: A kinematic study on healthy subjects. Journal of Neurophysiology, 81, 1685-1699.

Goldman, S.M., \& Tanner, C. (1998). Etiology of Parkinson's disease. In J. Jankovic \& E. Tolosa (Eds.) Parkinson's disease and movement disorders (pp.133-158) $\left(3^{\text {rd }}\right.$ ed.). Baltimore: Williams \& Wilkins.

Grosjean, F., Lane, H. (1973). Perception of reading rate by speakers and listeners. Journal of Experimental Psychology, 97(2), 141-147 
Haahr, B. (1998). Random sequence generator. Retrieved from:

http://www.random.org/sequences/

Hamani, C., \& Lozano, A.M. (2003). Physiology and pathophysiology of Parkinson's disease. Ann N Y Academy of Science, 991, 15-21.

Healey, C., Jones, R., Berky, R. (1997). Effects of perceived listeners on speakers' vocal intensity. Journal of Voice, 11(1), 67-73

Ho, A.K., Bradshaw, J.L., \& Iansek, R. (2000). Volume perception in parkinsonian speech. Movement Disorders, 15, 1125-1131.

Ho, A.K., Bradshaw, J.L., Iansek, R., \& Alfredson, R. (1999a). Speech volume regulation in Parkinson's disease: effects of implicit cues and explicit instructions. Neuropsychologia, 37, 1453-1460.

Ho, A.K., Iansek, R., \& Bradshaw, J.L. (1999b). Regulation of parkinsonian speech volume: The effect of interlocutor distance. Journal of Neurology, Neurosurgery, and Psychiatry, 67, 199-202.

Ho, A.K., Iansek R., \& Bradshaw, J.L. (2002). The effect of a concurrent task on Parkinsonian speech. Journal of Clinical and Experimental Neurophysiology, 24(1): 36-47.

Hoehn, M.M., Yahr, M.D. (1967). Parkinsonism: onset, progression, and mortality. Neurology, 17(5), 427-442.

Holmes, J.D., Jenkins, Johnson, A., Adams, S.G., Spaulding. (2010). Dual-task interference: The effects of verbal cognitive tasks on upright postural stability in Parkinson's disease. SAGE-Hindawi Publishing Corporation. doi:10.4061/2010/696492 
Kang, G.A., Bronstein, J.M., Masterman, D.L., Redelings, M., Crum, J.A., \& Ritz, B. (2005). Clinical characteristics in early Parkinson's disease in a central California population-based study. Movement Disorders, 20(9), 1133-42

Kelly, V.W., Eusterbrock, A.J., Shumway-Cook, A. (2011). A review of dual-task walking deficits in people with Parkinson's disease: motor and cognitive contributions, mechanisms, and clinical implications. Parkinson's Disease, (doi: 10.1155/2012/918719), 1-14.

Kemper, S., Herman, R.E., Lian, C. (2003). The cost of doing two things at once for young and older adults: talking while walking, finger tapping, and ignoring speech or noise. Psychology and Aging, 18(2), 181-192

Knuttson, E. (1972). An analysis of parkinsonian gait. Brain 95, 475-86

Korell, M., \& Tanner, C.M. (2005). Epidemiology of Parkinson's disease. In M. Ebadi \& R. Pfeiffer (Eds.) Parkinson's disease, 39-50, Boca Raton: CRC Press.

Lane, H.L., Catania, A.C., Stevens, S.S. (1961). Voice level: autophonics scale, perceived loudness, and effects of sidetone. The Journal of the Acoustical Society of America, $33(2), 160-167$

Lim, L.I., Van Wegen, E. E., de Goede, C.J., Jones, D., Rochester, L., Hetherington, V., Nieuwboer, A., Willems, A.M., \& Kwakkel, G. (2005). Measuring gait and gaitrelated activities in Parkinson's patients own home environment: a reliability, responsiveness and feasibility study. Parkinsonism Related Disorders, 11(1), 19-24.

Lutz, K.C., \& Mallard, A.R. (1986). Disfluencies and rate of speech in young adult nonstutterers. Journal of Fluency Disorders, 11, 307-316 
Maggioni, M., Veicteinas, A., Rampichini, S., Ce, E., Nemni, R., Riboldazzi, G., Merati, G. (2011). Energy cost of spontaneous walking in Parkinson's disease patients. Neurological Sciences, doi: 10.1007/s10072-011-0827-6

Mak, M. (2013). Reduced step length, not step length variability is central to gait hypokinesia in people with Parkinson's disease. Clinical Neurology and Neurosurgery, 115, 587-590

McRae, P.A. \& Tjaden, K. (2002). Acoustic and perceptual consequences of articulatory rate change in Parkinson Disease. Journal of Speech, Language, and Hearing Research, 45, 35-50

Meinzer, M., Breitenstein, C., Westerhoff, U., Sommer, J., Rosser, N., Rodriguez, A.D., Harnish, S., Knecht, S., \& Floel, A. (2011). Motor cortex preactivation by standing facilitates word retrieval in aphasia. Neurorehabilitation and Neural Repair, 25(2): 178-187.

Michael, D.D., Siegel, G.M., \& Pick, H.L. (1995). Effects of distance on vocal intensity. Journal of Speech and Hearing Research, 38, 1176-1183.

Miller, N., Noble, E., Jones, D. (2006). Life with communication changes in Parkinson's disease. Age and Ageing, 35(3), 235-239.

Morris, M.E., Iansek, R., Matyas, T.A., Summers, J.J. (1994a). The pathogenesis of gait hypokinesia in Parkinson's disease. Brain 117, 1169-81

Morris, M.E., Iansek, R., Matyas, T.A., Summers, J.J. (1994b). Ability to modulate walking cadence remains intact in Parkinson's disease. Journal of Neurology, Neurosurgery \& Psychiatry, 57(12), 1532-34 
Morris, M.E., Iansek, R., Matyas, T.A., Summers, J.J. (1996). Stride length regulation in Parkinson's disease normalization strategies and underlying mechanisms. Brain, 199(2), 551-8

Murray, M.P., Sepic, S.B., Gena, M., Gardner, G.M., \& Downs, J. (1978). Walking patterns of men with Parkinsonism. American Journal of Physical Medicine, 57(6), 278-294

O'Shea, S., Morris, M.E., Iansek, R. (2002). Dual-task interference during gait in people with Parkinson's disease: effects of motor versus cognitive secondary tasks. Journal of the American Physical Therapy Association, 82, 888-897

Pohl, P.S., Kemper, S., Siengsukon, C.F., Boyd, L., Vidoni, E., \& Herman, R.E. Older adults with and without stroke reduce cadence to meet the demands of talking (2011) Journal of Geriatric Physical Therapy, 34(1), 35-40.

Post, B., Merkus, P., J. de Haan, R., Speelman, J.D. (2007). Prognostic factors for the progression of Parkinson's disease: a systematic review. Movement Disorders, 22(13), 1839-51

Ramig, L., Sapir, S., Fox, C., \& Countryman, S. (2001). Changes in vocal loudness following intensive voice treatment (LSVT) in individuals with Parkinson's disease: a comparison with untreated patients and normal age-matched controls. Movement Disorders, 16, 79-83

Rochester, L., Hetherington, V., Jones, D., Nieuwboer, A., Willems, AM., Kwakkel, G., Van Wegen, E. (2004). Attending to the task: interference effects of functional tasks on walking in Parkinson's disease and the roles of cognition, depression, fatigue, and balance. Archives of Physical Medicine and Rehabilitation, 85, 157885 
Rochester, L., Hetherington, V., Jones, D., Nieuwboer, A., Willems, AM., Kwakkel, G., Van Wegen, E. (2005). The effect of external rhythmic cues (auditory and visual) on walking during a functional task in homes of people with Parkinson's disease. Archives of Physical Medicine and Rehabilitation, 86, 999-107

Rochester, L., Nieuwboer, A., Baker, K. Hetherington, V., Willems, A., Kwakkel, G., Wegen, E.V., Lim, I., Jones, D. (2008). Walking speed during single and dual tasks in Parkinson's disease: which characteristics are important? Movement Disorders, 23(16), 2312-2318.

Sapir, S., Spielman, J.L., Ramig, L.O., Story, B.H., Fox, C. (2007). Effects of intensive voice treatment (the Lee Silverman voice treatment [lsvt]) on vowel articulation in dysarthric individuals with idiopathic Parkinson disease: acoustic and perceptual findings. Journal of Speech, Language, and Hearing Research, 50, 899-912.

Schulman, R. (1989). Articulatory dynamics of loud and normal speech. Journal of the Acoustical Society of America, 85(1), 295-312.

Schrag, A., Jahanshahi, M., \& Quinn, N. (2000). What contributes to quality of life in patients with Parkinson's disease? Journal of Neurology, Neurosurgery, and Psychiatry, 69(3), 308-12

Seitz, R.J., \& Roland, P.E. (1992). Learning of sequential finger movements in man: a combined kinematic and positron emission tomography (PET) study. European Journal of Neuroscience, 4, 154-165.

Sheridan MR, Flowers KA, \& Hurrell J. (1987). Programming and execution of movement in Parkinson's disease. Brain, 110, 1247-71

Stevens, S.S. (1962). The surprising simplicity of sensory metrics. American Psychologist, 17, 29-39 
Tan, D., Danoudis, M., McGinley, J., Morris, M, E. (2012). Relationships between motor aspects of gait impairments and activity limitations in people with Parkinson's disease: A systematic review. Parkinsonism and Related Disorders, 18, 117-124

Tanner, C.M., Goldman, S.M. (1996). Epidemiology of Parkinson's disease. Neurologic Clinics, 14 (2), 317-35

Tjaden, K., Wilding, G. (2011). Effects of speaking task on intelligibility in Parkinson's disease. Clinical Linguistics \& Phonetics, 25(2), 155-68

Walsh, B., Smith, A. (2012). Basic parameters of articulatory movements and acoustics in individuals with Parkinson's disease. Movement Disorders 27(7), 843-50

Venkatagiri, H.S. (1999). Clinical measurement of rate of reading and discourse in young adults. Journal of Fluency Disorders, 24, 209-226

Weiner, W.J., \& Lang, A.E. (1989). Movement disorders: A comprehensive survey. Mount Kisko: Futura.

Weismer, G., Jeng, J-Y., Laures, J., Kent R.D., \& Kent, J.F. (2001). Acoustic and intelligibility characteristics of sentence production in neurogenic speech disorders. Folia Phoniatrica et Logopaedica, 53, 1-18

Wenke, R.J., Theodoros, D., Cornwell, P. (2011). A comparison of the effects of the Lee Silverman voice treatment and traditional therapy on intelligibility, perceptual speech features, and everyday communication in nonprogressive dysarthria. Journal of Medical Speech-Language Pathology, 19(4), 1-24.

Wirdefelt, K., Adami, H., Cole, P., Trichopoulos, D., \& Mandel, J. (2011). Epidemiology and etiology of Parkinson's disease: a review of the evidence. European Journal of 
Epidemiology, 26, 1-58.

Wu, T., \& Hallet, M. (2009). Dual-task interference in Parkinson's disease. European Neurological Review, 34-37.

Yogev, G., Giladi, N., Peretz, C., Springer, S., Simon, E.S., Hausdorff, J.M. (2005). Dual tasking, gait rhythmicity, and Parkinson's disease: which aspects of gait are attention demanding? European Journal of Neuroscience, 22(5): 1248-1256

Yorkston, K.M., Hammen, V.L., Beukelman, D.R. (1990). The effect of rate control on the intelligibility and naturalness of dysarthric speech. Journal of Speech and Hearing Disorders, 55, 550-60

Zahorik, P. \& Kelly, J.W. (2007). Accurate vocal compensation for sound intensity loss with increasing distance in natural environments. Journal of the Acoustical Society of America, 122(5), 143-150 


\title{
Appendices
}

\section{Appendix A. Western University Ethics Approval}

\author{
Principal Investigator: Dr. Scott Adams \\ File Number: 102929 \\ Review Level:Delegated \\ Approved Local Adult Participants: 40 \\ Approved Local Minor Participants:0 \\ Protocol Title:Effect of concurrent walking tasks and interlocutor distance on conversational speech in Parkinson's \\ disease. \\ Department \& Institution:Health SciencesiCommunication Sciences \& Disorders, Western University \\ Sponsor: \\ Ethics Approval Date:September 06, 2012 Expiry Date:August 31, 2013 \\ Documents Reviewed \& Approved \& Documents Received for Information:

\begin{tabular}{|l|l|l|}
\hline Document Name & Comments & Version Date \\
\hline Western University Protocol & & \\
\hline Letter of Information & Controls & $2012 / 07 / 25$ \\
\hline Other & Consent Form & $2012 / 07 / 25$ \\
\hline Letter of Information & Participants with Parkinson's disease & $2012 / 07 / 25$ \\
\hline
\end{tabular}

This is to notify you that The University of Western Ontario Research Ethics Board for Health Sciences Research Involving Human Subjects (HSREB) which is organized and operates according to the Tri-Council Policy Statement: Ethical Conduct of Research Involving Humans and the Health Canada/lCH Good Clinical Practice Practices: Consolidated Guidelines; and the applicable laws and regulations of Ontario has reviewed and granted approval to the above referenced revision(s) or amendment(s) on the approval date noted above. The membership of this REB also complies with the membership requirements for REB's as defined in Division 5 of the Food and Drug Regulations.

The ethics approval for this study shall remain valid until the expiry date noted above assuming timely and acceptable responses to the HSREB's periodic requesis for surveillance and monitoring information. If you require an updated approval notice prior to that time you must request it using the University of Western Ontario Updated Approval Request Form

Members of the HSREB who are named as investigators in research studies, or declare a conflict of interest, do not participate in discussion related to, nor vote on, such studies when they are presented to the HSREB.

The Chair of the HSREB is Dr. Joseph Gilbert. The HSREB is registered with the U.S. Department of Health \& Human Services under the IRB registration number IRB 00000940.

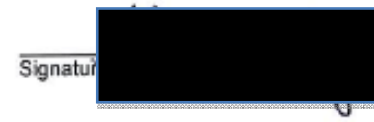

\begin{tabular}{|c|c|c|}
\hline 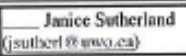 & $\begin{array}{l}\text { Grace Kelly } \\
\text { facc.kelly (r) uwoca) }\end{array}$ & \begin{tabular}{|l} 
Shantel Walcolt \\
(swalcon \\
\end{tabular} \\
\hline
\end{tabular}

This is an official document. Please retain the original in your fies. 


\title{
Appendix B. PD Participant Letter of Information
}

\author{
LETTER OF INFORMATION \\ Participants with Parkinson's disease
}

\begin{abstract}
STUDY TITLE
Effects of concurrent walking tasks and interlocutor distance on conversational speech in Parkinson's disease.
\end{abstract}

\author{
PRINCIPAL INVESTIGATOR \\ Scott Adams, Ph.D. \\ Professor \\ School of Communication Sciences and Disorders; Clinical Neurological Sciences \\ University of Western Ontario
}

\section{CO-INVESTIGATORS}

Allyson Dykstra, Ph.D.

Assistant Professor

School of Communication Sciences and Disorders

University of Western Ontario

Dr. Mandar Jog, MD, FRCPC

Director, Movement Disorders Program,

London Health Sciences Centre, University Campus and

University of Western Ontario

Cassandra McCaig

MSc. Candidate,

Health and Rehabilitation Sciences

University of Western Ontario

\section{INTRODUCTION}

This letter of information describes a research study and what you may expect if you decide to participate. You should read the letter carefully and ask the person discussing this with you any questions that you may have before making a decision whether or not to participate. This form contains important information and telephone numbers, so you should keep this copy for future reference. If you decide not to participate in this study, the decision will not be held against you and will not affect your treatment in any way.

You are being asked to participate in this research study because you are an individual with reduced speech intensity and Parkinson's disease. The purpose of this study is to investigate the effects of various concurrent tasks and interlocutor distance on speech parameters in Parkinson's disease. An example of tasks being performed concurrently is speaking while talking. 
This study will involve 40 participants. Twenty of the participants will have reduced speech intensity and Parkinson's disease. The other twenty participants will not have any neurological conditions. Information about participants will be collected from patient charts and person-to-person interviews by the principal experimenter or another designated member of the research team. This will include information about the participant's date of birth, general medical history, neurological history, and speech and hearing history.

In this study, you will be asked to perform a variety of separate and concurrent speech and walking tasks for a total of 13 experimental conditions. The experimental conditions involve evaluating several acoustic parameters of speech in isolation, while walking, and at an interlocutor distance of 1-metre or 6-metres. The acoustic measures will include average speech rate and average speech intensity. Speech tasks will involve engaging in a conversation with the experimenter for approximately 2-3 minutes about a familiar topic. The conversational topics will include favourite vacations, interests, hobbies, relatives, occupational experiences, etc . The conversations will take place with the experimenter positioned at either a 1-meter or 6-meter interlocutor distance from you. The gait measures include a stride length and walking rate. The walking tasks will involve 5 different tasks. These include 1) sitting, 2) standing, 3) walking at a normal or habitual speed, 4) walking at a speed that is self-perceived to be two times slower than the habitual speed, and 5) walking at a speed that is self-perceived to be two times faster than habitual. During all of the conditions, you will wear a headset microphone that will record the speech on a laptop computer. After you complete the experimental trials, we will conduct a standard hearing assessment. During the standard hearing assessment, you will hear a variety of sounds at different intensities and frequencies. If you agree to participate you will be asked to come one time to Althouse College at the University of Western Ontario for testing. It is anticipated that the total time for this experiment and the hearing test will be no more than 90 minutes.

The experimental procedures will require very brief and intermittent physical effort, and there is no known discomfort or risk involved in performing them. You will be asked to walk down and back a 25-metre corridor and you will be given rest breaks approximately every five minutes or more frequently if required.

The procedures that will be used during this study are experimental in nature and will not provide any direct benefit to the participant's medical condition, however, it is anticipated that this research will provide new information about the effects of conversational speech produced at different loudness levels on walking efficiency and gait symptom severity in $\mathrm{PD}$, and that these results will have important implications for the development of new speech and gait therapy programs in PD. Financial compensation will not be provided upon completion of this study. Free parking will be provided while you are visiting the gymnasium at Althouse College.

Participation in this study is voluntary. You may refuse to participate, refuse to answer any questions, or withdraw from the study at any time with no effect on your future care. 
All of the information obtained in this study will be held in strict confidence. Your name and any identifying information will be removed from the data. If the results of the study are published, your name will not be used and no information that discloses your identity will be released or published.

Throughout the study, all confidential information will be preserved in a locked filing cabinet in the Principal Investigator's laboratory at Elborn College, University of Western Ontario.

If requested, you will be provided with a copy of any publication related to the results of this study when it becomes available.

If you have any questions or would like additional information about this study, please contact Professor Scott Adams at the

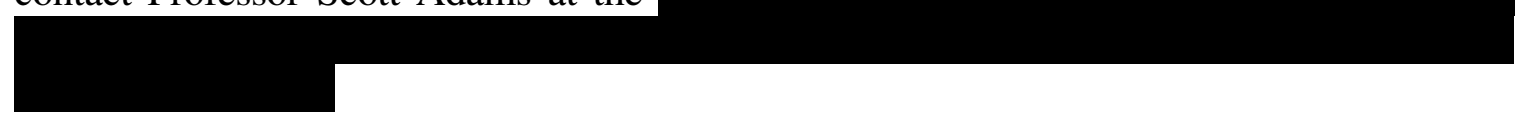

If you have any questions about the conduct of this study or your rights as a research subject you may contact Dr. David Hill, Scientific Director, Lawson Health Research Institute, at

If you agree to participate in this study, please sign the consent form on the next page.

Sincerely,

Scott Adams, Ph.D.

Professor 


\title{
Appendix C. Control Participant Letter of Information
}

\section{LETTER OF INFORMATION \\ Control Participants}

\author{
STUDY TITLE \\ Effects of concurrent walking tasks and interlocutor distance on conversational speech in \\ Parkinson's disease.
}

\section{PRINCIPAL INVESTIGATOR}

Scott Adams, Ph.D.

Professor

School of Communication Sciences and Disorders; Clinical Neurological Sciences

University of Western Ontario

\section{CO-INVESTIGATORS}

Allyson Dykstra, Ph.D.

Assistant Professor

School of Communication Sciences and Disorders

University of Western Ontario

Dr. Mandar Jog, MD, FRCPC

Director, Movement Disorders Program,

London Health Sciences Centre, University Campus and

University of Western Ontario

Cassandra McCaig

MSc. Candidate,

Health and Rehabilitation Sciences

University of Western Ontario

\section{INTRODUCTION}

This letter of information describes a research study and what you may expect if you decide to participate. You should read the letter carefully and ask the person discussing this with you any questions that you may have before making a decision whether or not to participate. This form contains important information and telephone numbers, so you should keep this copy for future reference. If you decide not to participate in this study, the decision will not be held against you and will not affect your treatment in any way.

The purpose of this study is to investigate the effects of various concurrent tasks and interlocutor distance on speech parameters in Parkinson's disease. An example of tasks being performed concurrently is talking while walking.

This study will involve 40 participants. Twenty of the participants will have reduced speech intensity and Parkinson's disease. The other twenty participants will not have any 
neurological conditions. Information about participants will be collected from patient charts and person-to-person interviews by the principal experimenter or another designated member of the research team. This will include information about the participant's date of birth, general medical history, neurological history, and speech and hearing history.

In this study, you will be asked to perform a variety of separate and concurrent speech and walking tasks for a total of 13 experimental conditions. The experimental conditions involve evaluating several acoustic parameters of speech in isolation, while walking, and at an interlocutor distance of 1-metre or 6-metres. The acoustic measures will include average speech rate and average speech intensity. Speech tasks will involve engaging in a conversation with the experimenter for approximately 2-3 minutes about a familiar topic. The conversational topics will include favourite vacations, interests, hobbies, relatives, occupational experiences, etc. The conversations will take place with the experimenter positioned at either a 1-meter or 6-meter interlocutor distance from you. The gait measures include a stride length and walking rate. The walking tasks will involve 5 different tasks. These include 1) sitting, 2) standing, 3) walking at a normal or habitual speed, 4) walking at a speed that is self-perceived to be two times slower than the habitual speed, and 5) walking at a speed that is self-perceived to be two times faster than habitual. During all of the conditions, you will wear a headset microphone that will record the speech on a laptop computer. After you complete the experimental trials, we will conduct a standard hearing assessment. During the standard hearing assessment, you will hear a variety of sounds at different intensities and frequencies. If you agree to participate you will be asked to come one time to Althouse College at the University of Western Ontario for testing. It is anticipated that the total time for this experiment and the hearing test will be no more than 90 minutes.

The experimental procedures will require very brief and intermittent physical effort, and there is no known discomfort or risk involved in performing them. You will be asked to walk down and back a 25-metre corridor and you will be given rest breaks approximately every five minutes or more frequently if required.

The procedures that will be used during this study are experimental in nature and will not provide any direct benefit to the participant's medical condition, however, it is anticipated that this research will provide new information about the effects of conversational speech produced at different loudness levels on walking efficiency and gait symptom severity in $\mathrm{PD}$, and that these results will have important implications for the development of new speech and gait therapy programs in PD. Financial compensation will not be provided upon completion of this study. Free parking will be provided while you are visiting the lab at Elborn College.

Participation in this study is voluntary. You may refuse to participate, refuse to answer any questions, or withdraw from the study at any time with no effect on your future care.

All of the information obtained in this study will be held in strict confidence. Your name and any identifying information will be removed from the data. If the results of the study 
are published, your name will not be used and no information that discloses your identity will be released or published.

Throughout the study, all confidential information will be preserved in a locked filing cabinet in the Principal Investigator's laboratory at Elborn College, University of Western Ontario.

If requested, you will be provided with a copy of any publication related to the results of this study when it becomes available.

If you have any questions or would like additional information about this study, please contact Professor Scott Adams at the

If you have any questions about the conduct of this study or your rights as a research subject you may contact Dr. David Hill, Scientific Director, Lawson Health Research Institute, at

If you agree to participate in this study, please sign the consent form on the next page.

Sincerely,

Scott Adams, Ph.D.

Professor 


\section{Appendix D. Participant Consent Form}

\section{CONSENT FORM \\ Participant with Parkinson's disease}

\section{STUDY TITLE}

Effects of concurrent walking tasks and interlocutor distance on conversational speech in Parkinson's disease.

\section{PRINCIPAL INVESTIGATOR}

Scott Adams, Ph.D.

Professor

School of Communication Sciences and Disorders; Clinical Neurological Sciences

Western University

\section{CO-INVESTIGATORS}

Allyson Dykstra, Ph.D.

Assistant Professor

School of Communication Sciences and Disorders

Western University

Dr. Mandar Jog, MD, FRCPC

Director, Movement Disorders Program,

London Health Sciences Centre, University Campus and

Western University

Cassandra McCaig

MSc. Candidate,

Health and Rehabilitation Sciences

Western University

I have read the Letter of Information (have had the nature of the study explained to me), and I agree to participate. All questions have been answered to my satisfaction.

\begin{tabular}{lll}
\hline Signature of Research Subject & Printed Name
\end{tabular}




\section{Appendix E. Participant Intake Survey}

\section{Parkinson's Speech Study}

Section 1: Demographic Information

Name:

Phone Number:

Birth Date (MM/DD/YYYY): Age:

Occupation:

Gender: $\quad$ Male Female

Parkinson's Disease $\square \quad$ Control $\square \quad$ Date of Diagnosis:

Time since last PD medication:

Time until next PD medication:

Type of medication: Sinemet $\square$

Other $\square$ :

Section 2: Hearing Screening

Hearing Threshold:

\begin{tabular}{|c|c|c|}
\hline & Right & Left \\
\hline $500 \mathrm{~dB}$ & & \\
\hline $1000 \mathrm{~dB}$ & & \\
\hline $2000 \mathrm{~dB}$ & & \\
\hline $4000 \mathrm{~dB}$ & & \\
\hline
\end{tabular}

Section 3: Speech, Language, Hearing or Neurological Impairment

Ever been diagnosed with a speech impairment? Yes $\square$ No

If yes, please indicate the diagnosis:

Ever been diagnosed with a language impairment? Yes No

If yes, please indicate the diagnosis:

Ever been diagnosed with a hearing impairment? Yes No

If yes, please indicate the diagnosis:

Ever been diagnosed with a neurological impairment? Yes $\square$ No If yes, please indicate the diagnosis: 


\section{Appendix F. 3 Way ANOVA: Speech Intensity}

\section{General Linear Model}

Index for 3 Way ANOVA: Speech Intensity

$*$ code $1=$ control participants, code $2=$ PD participants, interloc $1=$ one meter interlocutor distance, interloc $2=$ six meters interlocutor distance, walking $1=$ sitting, walking $2=$ standing, walking $3=$ normal walking speed, walking $4=$ fast walking speed, walking $5=$ slow walking speed, $\mathrm{c} 1=$ sitting at one meter interlocutor distance, $\mathrm{c} 2=$ sitting at six meters interlocutor distance, $\mathrm{c} 3=$ standing at one meter interlocutor distance, $\mathrm{c} 4=$ standing at six meters interlocutor distance, $\mathrm{c} 6=$ walking normal speed at one meter interlocutor distance, $\mathrm{c} 7=$ walking normal speed at six meters interlocutor distance, $\mathrm{c} 8=$ walking fast speed at one meter interlocutor distance, $\mathrm{c} 9=$ walking fast speed at six meters interlocutor distance, $\mathrm{c} 10=$ walking slow speed at one meter interlocutor distance, $\mathrm{c} 11=$ walking fast speed at six meters interlocutor distance.

Within-Subjects Factors

Measure: MEASURE_1

\begin{tabular}{|ll|l|}
\hline walking & interloc & $\begin{array}{c}\text { Dependent } \\
\text { Variable }\end{array}$ \\
\hline 1 & 1 & c1 \\
& 2 & c2 \\
\hline 2 & 1 & c3 \\
& 2 & c4 \\
\hline 3 & 1 & $c 6$ \\
& 2 & $c 7$ \\
\hline 4 & 1 & $c 8$ \\
& 2 & $c 9$ \\
\hline 5 & 1 & $c 10$ \\
& 2 & $c 11$ \\
\hline
\end{tabular}

Between-Subjects

Factors

\begin{tabular}{|ll|r|}
\hline & & N \\
\hline code & 1.00 & 12 \\
& 2.00 & 11 \\
\hline
\end{tabular}


Descriptive Statistics

\begin{tabular}{|c|c|c|c|c|}
\hline & code & Mean & Std. Deviation & $\mathrm{N}$ \\
\hline \multirow[t]{3}{*}{$\mathrm{c} 1$} & 1.00 & 69.4210 & 2.66514 & 12 \\
\hline & 2.00 & 65.2371 & 3.92034 & 11 \\
\hline & Total & 67.4200 & 3.88638 & 23 \\
\hline \multirow[t]{3}{*}{$\mathrm{c} 2$} & 1.00 & 72.8396 & 2.40087 & 12 \\
\hline & 2.00 & 68.5264 & 3.96342 & 11 \\
\hline & Total & 70.7767 & 3.85688 & 23 \\
\hline \multirow[t]{3}{*}{ c3 } & 1.00 & 69.1825 & 2.87194 & 12 \\
\hline & 2.00 & 64.5422 & 4.11779 & 11 \\
\hline & Total & 66.9632 & 4.17715 & 23 \\
\hline \multirow[t]{3}{*}{$c 4$} & 1.00 & 71.9051 & 2.54040 & 12 \\
\hline & 2.00 & 67.9904 & 3.68067 & 11 \\
\hline & Total & 70.0328 & 3.65821 & 23 \\
\hline \multirow[t]{3}{*}{ c6 } & 1.00 & 71.7666 & 2.61830 & 12 \\
\hline & 2.00 & 68.3313 & 2.91354 & 11 \\
\hline & Total & 70.1236 & 3.21943 & 23 \\
\hline \multirow[t]{3}{*}{ c7 } & 1.00 & 74.1567 & 2.53170 & 12 \\
\hline & 2.00 & 69.7401 & 3.36734 & 11 \\
\hline & Total & 72.0444 & 3.66706 & 23 \\
\hline \multirow[t]{3}{*}{$\mathrm{c} 8$} & 1.00 & 73.2815 & 3.07382 & 12 \\
\hline & 2.00 & 70.0443 & 3.34023 & 11 \\
\hline & Total & 71.7333 & 3.53969 & 23 \\
\hline \multirow[t]{3}{*}{$\mathrm{c9}$} & 1.00 & 75.2814 & 3.01434 & 12 \\
\hline & 2.00 & 70.4959 & 3.45738 & 11 \\
\hline & Total & 72.9927 & 3.99383 & 23 \\
\hline \multirow[t]{3}{*}{ c10 } & 1.00 & 71.3295 & 2.52453 & 12 \\
\hline & 2.00 & 67.0250 & 4.96253 & 11 \\
\hline & Total & 69.2708 & 4.38341 & 23 \\
\hline \multirow[t]{3}{*}{ c11 } & 1.00 & 73.9377 & 2.97198 & 12 \\
\hline & 2.00 & 69.7526 & 3.54749 & 11 \\
\hline & Total & 71.9361 & 3.83479 & 23 \\
\hline
\end{tabular}


Tests of Within-Subjects Effects

Measure: MEASURE_1

\begin{tabular}{|c|c|c|c|c|c|}
\hline Source & & $\begin{array}{c}\text { Type III Sum } \\
\text { of Squares }\end{array}$ & df & Mean Square & $\mathrm{F}$ \\
\hline \multirow[t]{4}{*}{ walking } & Sphericity Assumed & 444.592 & 4 & 111.148 & 58.406 \\
\hline & Greenhouse-Geisser & 444.592 & 2.945 & 150.979 & 58.406 \\
\hline & Huynh-Feldt & 444.592 & 3.640 & 122.127 & 58.406 \\
\hline & Lower-bound & 444.592 & 1.000 & 444.592 & 58.406 \\
\hline \multirow{4}{*}{ walking ${ }^{*}$ code } & Sphericity Assumed & 1.194 & 4 & .299 & .157 \\
\hline & Greenhouse-Geisser & 1.194 & 2.945 & .406 & .157 \\
\hline & Huynh-Feldt & 1.194 & 3.640 & .328 & .157 \\
\hline & Lower-bound & 1.194 & 1.000 & 1.194 & .157 \\
\hline \multirow[t]{4}{*}{ Error(walking) } & Sphericity Assumed & 159.853 & 84 & 1.903 & \\
\hline & Greenhouse-Geisser & 159.853 & 61.839 & 2.585 & \\
\hline & Huynh-Feldt & 159.853 & 76.449 & 2.091 & \\
\hline & Lower-bound & 159.853 & 21.000 & 7.612 & \\
\hline \multirow[t]{4}{*}{ interloc } & Sphericity Assumed & 343.506 & 1 & 343.506 & 103.233 \\
\hline & Greenhouse-Geisser & 343.506 & 1.000 & 343.506 & 103.233 \\
\hline & Huynh-Feldt & 343.506 & 1.000 & 343.506 & 103.233 \\
\hline & Lower-bound & 343.506 & 1.000 & 343.506 & 103.233 \\
\hline \multirow[t]{4}{*}{ interloc ${ }^{*}$ code } & Sphericity Assumed & 1.888 & 1 & 1.888 & .567 \\
\hline & Greenhouse-Geisser & 1.888 & 1.000 & 1.888 & .567 \\
\hline & Huynh-Feldt & 1.888 & 1.000 & 1.888 & .567 \\
\hline & Lower-bound & 1.888 & 1.000 & 1.888 & .567 \\
\hline \multirow[t]{4}{*}{ Error(interloc) } & Sphericity Assumed & 69.877 & 21 & 3.327 & \\
\hline & Greenhouse-Geisser & 69.877 & 21.000 & 3.327 & \\
\hline & Huynh-Feldt & 69.877 & 21.000 & 3.327 & \\
\hline & Lower-bound & 69.877 & 21.000 & 3.327 & \\
\hline \multirow[t]{4}{*}{ walking " interloc } & Sphericity Assumed & 35.239 & 4 & 8.810 & 4.067 \\
\hline & Greenhouse-Geisser & 35.239 & 2.445 & 14.411 & 4.067 \\
\hline & Huynh-Feldt & 35.239 & 2.923 & 12.054 & 4.067 \\
\hline & Lower-bound & 35.239 & 1.000 & 35.239 & 4.067 \\
\hline \multirow[t]{4}{*}{ walking ${ }^{*}$ interloc ${ }^{*}$ code } & Sphericity Assumed & 9.354 & 4 & 2.338 & 1.080 \\
\hline & Greenhouse-Geisser & 9.354 & 2.445 & 3.825 & 1.080 \\
\hline & Huynh-Feldt & 9.354 & 2.923 & 3.200 & 1.080 \\
\hline & Lower-bound & 9.354 & 1.000 & 9.354 & 1.080 \\
\hline \multirow[t]{4}{*}{ Error(walking"interloc) } & Sphericity Assumed & 181.963 & 84 & 2.166 & \\
\hline & Greenhouse-Geisser & 181.963 & 51.351 & 3.544 & \\
\hline & Huynh-Feldt & 181.963 & 61.390 & 2.964 & \\
\hline & Lower-bound & 181.963 & 21.000 & 8.665 & \\
\hline
\end{tabular}

Tests of Between-Subjects Effects

Measure: MEASURE_1

Transformed Variable: Average

\begin{tabular}{|l|r|r|r|r|r|r|}
\hline Source & $\begin{array}{c}\text { Type III Sum } \\
\text { of Squares }\end{array}$ & \multicolumn{1}{c|}{ df } & Mean Square & \multicolumn{1}{c|}{ F } & \multicolumn{1}{c|}{ Sig. } & \multicolumn{1}{c|}{$\begin{array}{c}\text { Partial Eta } \\
\text { Squared }\end{array}$} \\
\hline Intercept & 1132574.869 & 1 & 1132574.869 & 13025.686 & .000 & .998 \\
code & 984.432 & 1 & 984.432 & 11.322 & .003 & .350 \\
Error & 1825.936 & 21 & 86.949 & & & \\
\hline
\end{tabular}




\section{Appendix G. T-Test: Effect of Walking Speed on Speech Intensity}

\section{T-Test}

Index for T-Tests

c6-c11 = control participants, $c 6 p-c 11 p=$ PD participants, $c 6=$ normal walking speed at one meter interlocutor distance, $\mathrm{c} 7=$ normal walking speed at six meters interlocutor distance, $\mathrm{c} 8=$ fast walking speed at one meter interlocutor distance, $c 9=$ fast walking speed at six meters interlocutor distance, $\mathrm{c} 10=$ slow walking speed at one meter interlocutor distance, $\mathrm{c} 11=$ slow walking speed at six meters interlocutor distance, $c 6 p=$ normal walking speed at one meter interlocutor distance, $\mathrm{c} 7 \mathrm{p}=$ normal walking speed at six meters interlocutor distance, $c 8 p=$ fast walking speed at one meter interlocutor distance, $c 9 p=$ fast walking speed at six meters interlocutor distance, $\mathrm{c} 10 \mathrm{p}=$ slow walking speed at one meter interlocutor distance, $\mathrm{c} 11 \mathrm{p}=$ slow walking speed at six meters interlocutor distance

Paired Samples Statistics

\begin{tabular}{|ll|l|r|r|r|}
\hline & & Mean & $\mathrm{N}$ & Std. Deviation & $\begin{array}{c}\text { Std. Error } \\
\text { Mean }\end{array}$ \\
\hline Pair 1 & $c 6$ & 71.8541 & 14 & 2.45177 & .65526 \\
& $c 8$ & 73.4100 & 14 & 2.86963 & .76694 \\
Pair 2 & $c 6$ & 71.7666 & 12 & 2.61830 & .75584 \\
& $c 10$ & 71.3295 & 12 & 2.52453 & .72877 \\
Pair 3 & $c 8$ & 73.2815 & 12 & 3.07382 & .88733 \\
& $c 10$ & 71.3295 & 12 & 2.52453 & .72877 \\
Pair 4 & $c 7$ & 74.3042 & 13 & 2.48162 & .68828 \\
& $c 9$ & 75.4528 & 13 & 2.95140 & .81857 \\
Pair 5 & $c 7$ & 74.3042 & 13 & 2.48162 & .68828 \\
& $c 11$ & 73.9380 & 13 & 2.84545 & .78919 \\
Pair 6 & $c 9$ & 75.7291 & 14 & 3.01829 & .80667 \\
& $c 11$ & 74.1756 & 14 & 2.87479 & .76832 \\
Pair 7 & $c 6 p$ & 67.7637 & 13 & 3.01101 & .83510 \\
& $c 8 p$ & 69.7946 & 13 & 3.42679 & .95042 \\
Pair 8 & $c 6 p$ & 68.2381 & 15 & 3.25014 & .83918 \\
& $c 10 p$ & 67.6124 & 15 & 4.53329 & 1.17049 \\
Pair 9 & $c 8 p$ & 69.7946 & 13 & 3.42679 & .95042 \\
& $c 10 p$ & 67.1237 & 13 & 4.60416 & 1.27696 \\
Pair 10 & $c 7 p$ & 70.2812 & 14 & 3.18534 & .85132 \\
& $c 9 p$ & 71.0699 & 14 & 3.40757 & .91071 \\
Pair 11 & $c 7 p$ & 70.2812 & 14 & 3.18534 & .85132 \\
& $c 11 p$ & 70.3609 & 14 & 3.49535 & .93417 \\
Pair 12 & $c 9 p$ & 71.0699 & 14 & 3.40757 & .91071 \\
& $c 11 p$ & 70.3609 & 14 & 3.49535 & .93417 \\
\hline
\end{tabular}


Paired Samples Correlations

\begin{tabular}{|c|c|c|c|c|}
\hline & & $\mathrm{N}$ & Correlation & Sig. \\
\hline Pair 1 & $c 6 \&$ c8 & 14 & .903 & .000 \\
\hline Pair 2 & $c 6 \& c 10$ & 12 & .883 & .000 \\
\hline Pair 3 & c8 \& c10 & 12 & .871 & .000 \\
\hline Pair 4 & c7 \& c9 & 13 & .931 & .000 \\
\hline Pair 5 & c7 \& c11 & 13 & .810 & .001 \\
\hline Pair 6 & c9 \& c11 & 14 & .778 & .001 \\
\hline Pair 7 & $c 6 p \&$ c8p & 13 & .738 & .004 \\
\hline Pair 8 & $c 6 p \& c 10 p$ & 15 & .587 & .021 \\
\hline Pair 9 & $c 8 p \& c 10 p$ & 13 & .768 & .002 \\
\hline Pair 10 & $c 7 p \& c 9 p$ & 14 & .805 & .001 \\
\hline Pair 11 & $c 7 p \& c 11 p$ & 14 & .707 & .005 \\
\hline Pair 12 & $c 9 p \& c 11 p$ & 14 & .883 & .000 \\
\hline
\end{tabular}

Paired Samples Test

\begin{tabular}{|ll|r|r|r|}
\hline & & & & \\
& & & & \\
& & \multicolumn{1}{c|}{$\mathrm{t}$} & $\mathrm{df}$ & Sig. (2-tailed) \\
\hline Pair 1 & $\mathrm{c6}-\mathrm{c8}$ & -4.690 & 13 & .000 \\
Pair 2 & $\mathrm{c6-c10}$ & 1.212 & 11 & .251 \\
Pair 3 & $\mathrm{c8-c10}$ & 4.461 & 11 & .001 \\
Pair 4 & $\mathrm{c7}-\mathrm{c9}$ & -3.731 & 12 & .003 \\
Pair 5 & $\mathrm{c7}-\mathrm{c11}$ & .787 & 12 & .447 \\
Pair 6 & $\mathrm{c9-c11}$ & 2.956 & 13 & .011 \\
Pair 7 & $\mathrm{c6p}-\mathrm{c8p}$ & -3.098 & 12 & .009 \\
Pair 8 & $\mathrm{c6p}-\mathrm{c10p}$ & .652 & 14 & .525 \\
Pair 9 & $\mathrm{c8p}-\mathrm{c10p}$ & 3.266 & 12 & .007 \\
Pair 10 & $\mathrm{c7p}-\mathrm{c9p}$ & -1.426 & 13 & .177 \\
Pair 11 & $\mathrm{c7p}-\mathrm{c11p}$ & -.116 & 13 & .909 \\
Pair 12 & $\mathrm{c9p}-\mathrm{c11p}$ & 1.590 & 13 & .136 \\
\hline
\end{tabular}




\section{Appendix H. 3 Way ANOVA: Speech Rate}

\section{General Linear Model}

Index for 3 Way ANOVA: Speech Rate

*code $1=$ control participants, code $2=$ PD participants, interloc $1=$ one meter interlocutor distance, interloc $2=$ six meters interlocutor distance, walking $1=$ sitting, walking $2=$ standing, walking $3=$ normal walking speed, walking $4=$ fast walking speed, walking $5=$ slow walking speed, $\mathrm{cr} 1=$ sitting at one-meter, $\mathrm{cr} 2=$ sitting at six meters, $\mathrm{cr} 3=$ standing at one meter, $\mathrm{cr} 4=$ standing at six meters, $\mathrm{cr} 6=$ walking normal speed at one meter, $\mathrm{cr} 7=$ walking normal speed at six meters, $\operatorname{cr} 8=$ walking fast speed at one meter, $\operatorname{cr} 9=$ walking fast speed at six meters, $\operatorname{cr} 10=$ walking slow speed at one meter, cr11 = walking fast speed at six meters.

Within-Subjects Factors

Measure: MEASURE_1

\begin{tabular}{|ll|l|}
\hline walking & interloc & $\begin{array}{c}\text { Dependent } \\
\text { Variable }\end{array}$ \\
\hline 1 & 1 & $\mathrm{cr} 1$ \\
& 2 & $\mathrm{cr} 2$ \\
\hline 2 & 1 & $\mathrm{cr} 3$ \\
& 2 & $\mathrm{cr} 4$ \\
\hline 3 & 1 & $\mathrm{cr} 6$ \\
& 2 & $\mathrm{cr} 7$ \\
\hline 4 & 1 & $\mathrm{cr} 8$ \\
& 2 & $\mathrm{cr} 9$ \\
\hline 5 & 1 & $\mathrm{cr} 10$ \\
& 2 & $\mathrm{cr} 11$ \\
\hline
\end{tabular}

Between-Subjects

Factors

\begin{tabular}{|ll|r|}
\hline & & \multicolumn{1}{|c|}{$\mathrm{N}$} \\
\hline code & 1.00 & 11 \\
& 2.00 & 11 \\
\hline
\end{tabular}


Descriptive Statistics

\begin{tabular}{|ll|c|r|r|}
\hline & code & \multicolumn{1}{c|}{ Mean } & Std. Deviation & \multicolumn{1}{l|}{$\mathrm{N}$} \\
\hline cr1 & 1.00 & 229.6617 & 29.10693 & 11 \\
& 2.00 & 228.2927 & 38.93571 & 11 \\
& Total & 228.9772 & 33.55332 & 22 \\
\hline cr2 & 1.00 & 218.2083 & 23.28331 & 11 \\
& 2.00 & 232.4069 & 37.00249 & 11 \\
& Total & 225.3076 & 31.03130 & 22 \\
\hline cr3 & 1.00 & 227.0102 & 20.97546 & 11 \\
& 2.00 & 231.5191 & 36.30821 & 11 \\
& Total & 229.2646 & 29.02739 & 22 \\
\hline cr4 & 1.00 & 218.8889 & 38.98417 & 11 \\
& 2.00 & 231.5983 & 36.93138 & 11 \\
& Total & 225.2436 & 37.62303 & 22 \\
\hline cr6 & 1.00 & 228.8564 & 31.57892 & 11 \\
& 2.00 & 231.1968 & 38.51548 & 11 \\
& Total & 230.0266 & 34.39050 & 22 \\
\hline cr7 & 1.00 & 218.1605 & 21.61130 & 11 \\
& 2.00 & 239.9704 & 42.31409 & 11 \\
& Total & 229.0654 & 34.63518 & 22 \\
\hline
\end{tabular}

Descriptive Statistics

\begin{tabular}{|ll|c|r|r|}
\hline & c.nde & Mean & Std. Deviation & \multicolumn{1}{c|}{$\mathrm{N}$} \\
\hline cr8 & 1.00 & 226.5877 & 20.02412 & 11 \\
& 2.00 & 229.3937 & 43.31397 & 11 \\
& Total & 227.9907 & 32.96027 & 22 \\
\hline cr9 & 1.00 & 225.3152 & 25.35992 & 11 \\
& 2.00 & 235.5324 & 49.80557 & 11 \\
& Total & 230.4238 & 38.92077 & 22 \\
\hline cr10 & 1.00 & 220.2084 & 34.14003 & 11 \\
& 2.00 & 230.2061 & 47.46544 & 11 \\
& Total & 225.2073 & 40.66990 & 22 \\
\hline cr11 & 1.00 & 222.5544 & 33.90718 & 11 \\
& 2.00 & 226.6485 & 41.50489 & 11 \\
& Total & 224.6014 & 37.04291 & 22 \\
\hline
\end{tabular}


Tests of Within-Subjects Effects

Measure: MEASURE 1

\begin{tabular}{|c|c|c|c|c|c|}
\hline Source & & $\begin{array}{c}\text { Type III Sum } \\
\text { of Squares }\end{array}$ & df & Mean Square & $\mathrm{F}$ \\
\hline \multirow[t]{4}{*}{ walking } & Sphericity Assumed & 614.468 & 4 & 153.617 & .383 \\
\hline & Greenhouse-Geisser & 614.468 & 3.295 & 186.464 & .383 \\
\hline & Huynh-Feidt & 614.468 & 4.000 & 153.617 & .383 \\
\hline & Lower-bound & 614.468 & 1.000 & 614.468 & .383 \\
\hline \multirow[t]{4}{*}{ walking ${ }^{*}$ code } & Sphericity Assumed & 247.833 & 4 & 61.958 & .155 \\
\hline & Greenhouse-Geisser & 247.833 & 3.295 & 75.207 & .155 \\
\hline & Huynh-Feidt & 247.833 & 4.000 & 61.958 & .155 \\
\hline & Lower-bound & 247.833 & 1.000 & 247.833 & .155 \\
\hline \multirow[t]{4}{*}{ Error(walking) } & Sphericity Assumed & 32057.851 & 80 & 400.723 & \\
\hline & Greenhouse-Geisser & 32057.851 & 65.907 & 486.408 & \\
\hline & Huynh-Feldt & 32057.851 & 80.000 & 400.723 & \\
\hline & Lower-bound & 32057.851 & 20.000 & 1602.893 & \\
\hline \multirow[t]{4}{*}{ interloc } & Sphericity Assumed & 102.463 & 1 & 102.463 & .361 \\
\hline & Greenhouse-Geisser & 102.463 & 1.000 & 102.463 & .361 \\
\hline & Huynh-Feldt & 102.463 & 1.000 & 102.463 & .361 \\
\hline & Lower-bound & 102.463 & 1.000 & 102.463 & .361 \\
\hline \multirow[t]{4}{*}{ interloc ${ }^{*}$ code } & Sphericity Assumed & 1101.168 & 1 & 1101.168 & 3.876 \\
\hline & Greenhouse-Geisser & 1101.168 & 1.000 & 1101.168 & 3.876 \\
\hline & Huynh-Feidt & 1101.168 & 1.000 & 1101.168 & 3.876 \\
\hline & Lower-bound & 1101.168 & 1.000 & 1101.168 & 3.876 \\
\hline \multirow[t]{4}{*}{ Error(interioc) } & Sphericity Assumed & 5682.112 & 20 & 284.106 & \\
\hline & Greenhouse-Geisser & 5682.112 & 20.000 & 284.106 & \\
\hline & Huynh-Feidt & 5682.112 & 20.000 & 284.106 & \\
\hline & Lower-bound & 5682.112 & 20.000 & 284.106 & \\
\hline \multirow[t]{4}{*}{ walking ${ }^{*}$ interloc } & Sphericity Assumed & 302.836 & 4 & 75.709 & .160 \\
\hline & Greenhouse-Geisser & 302.836 & 3.360 & 90.136 & .160 \\
\hline & Huynh-Feidt & 302.836 & 4.000 & 75.709 & .160 \\
\hline & Lower-bound & 302.836 & 1.000 & 302.836 & .160 \\
\hline \multirow[t]{4}{*}{ walking ${ }^{*}$ interloc ${ }^{*}$ code } & Sphericity Assumed & 1039.541 & 4 & 259.885 & .549 \\
\hline & Greenhouse-Geisser & 1039.541 & 3.360 & 309.409 & .549 \\
\hline & Huynh-Feidt & 1039.541 & 4.000 & 259.885 & .549 \\
\hline & Lower-bound & 1039.541 & 1.000 & 1039.541 & .549 \\
\hline \multirow[t]{4}{*}{ Error(walking*interloc) } & Sphericity Assumed & 37901.922 & 80 & 473.774 & \\
\hline & Greenhouse-Geisser & 37901.922 & 67.195 & 564.057 & \\
\hline & Huynh-Feldt & 37901.922 & 80.000 & 473.774 & \\
\hline & Lower-bound & 37901.922 & 20.000 & 1895.096 & \\
\hline
\end{tabular}

Tests of Between-Subjects Effects

Measure: MEASURE_1

Transformed Variable: Average

\begin{tabular}{|l|r|r|r|r|r|r|}
\hline Source & $\begin{array}{c}\text { Type III Sum } \\
\text { of Squares }\end{array}$ & df & Mean Square & \multicolumn{1}{c|}{ F } & Sig. & $\begin{array}{c}\text { Partial Eta } \\
\text { Squared }\end{array}$ \\
\hline Intercept & 11397471.45 & 1 & 11397471.45 & 1281.903 & .000 & .985 \\
code & 3636.513 & 1 & 3636.513 & .409 & .530 & .020 \\
Error & 177821.105 & 20 & 8891.055 & & & \\
\hline
\end{tabular}




\section{Appendix I. 3 Way ANOVA: Walking Speed and Concurrent Talking}

\section{General Linear Model}

Index for 3 Way ANOVA: Walking Speed and Concurrent Talking

$*$ code $1=$ control participants, code $2=$ PD participants, speed $1=$ slow, speed $2=$ normal, speed $3=$ fast, talk $1=$ talking and walking, talk $2=$ walking only, slow $1=$ talking while walking at a slow speed, slowNT $=$ walking at slow speed without talking, norm $1=$ talking while walking at normal speed, normNT $=$ walking at normal speed without talking, fast $1=$ talking and walking at fast speed, fastNT $=$ walking at fast speed without talking

Within-Subjects Factors

Measure: MEASURE_1
\begin{tabular}{|ll|l|}
\hline SPEED & talk & $\begin{array}{c}\text { Dependent } \\
\text { Variable }\end{array}$ \\
\hline 1 & 1 & SLOW1 \\
& 2 & SLOWNT \\
\hline 2 & 1 & NORM1 \\
& 2 & NORMNT \\
\hline 3 & 1 & FAST1 \\
& 2 & FASTNT \\
\hline
\end{tabular}

Between-Subjects Factors

\begin{tabular}{|ll|r|}
\hline & & $\mathrm{N}$ \\
\hline CODE & 1.000 & 14 \\
& 2.000 & 14 \\
\hline
\end{tabular}




\section{Descriptive Statistics}

\begin{tabular}{|ll|r|r|r|}
\hline & CODE & \multicolumn{1}{|c|}{ Mean } & Std. Deviation & \multicolumn{1}{l|}{$\mathrm{N}$} \\
\hline SLOW1 & 1.000 & .83130 & .147963 & 14 \\
& 2.000 & .74857 & .192217 & 14 \\
& Total & .78994 & .173507 & 28 \\
\hline SLOWNT & 1.000 & .85528 & .166066 & 14 \\
& 2.000 & .86606 & .215004 & 14 \\
& Total & .86067 & .188589 & 28 \\
\hline NORM1 & 1.000 & 1.25887 & .189022 & 14 \\
& 2.000 & .94297 & .227029 & 14 \\
& Total & 1.10092 & .260560 & 28 \\
\hline NORMNT & 1.000 & 1.41014 & .122036 & 14 \\
& 2.000 & 1.14908 & .255108 & 14 \\
& Total & 1.27961 & .237013 & 28 \\
\hline FAST1 & 1.000 & 1.69295 & .145332 & 14 \\
& 2.000 & 1.23931 & .323536 & 14 \\
& Total & 1.46613 & .337523 & 28 \\
\hline FASTNT & 1.000 & 1.83987 & .151398 & 14 \\
& 2.000 & 1.45971 & .242523 & 14 \\
& Total & 1.64979 & .277172 & 28 \\
\hline
\end{tabular}


Tests of Within-Subjects Effects

Measure: MEASURE_1

\begin{tabular}{|c|c|c|c|c|c|}
\hline Source & & $\begin{array}{c}\text { Type III Sum } \\
\text { of Squares }\end{array}$ & $\mathrm{df}$ & Mean Square & $\mathrm{F}$ \\
\hline \multirow[t]{4}{*}{ SPEED } & Sphericity Assumed & 15.030 & 2 & 7.515 & 286.104 \\
\hline & Greenhouse-Geisser & 15.030 & 1.693 & 8.877 & 286.104 \\
\hline & Huynh-Feldt & 15.030 & 1.868 & 8.046 & 286.104 \\
\hline & Lower-bound & 15.030 & 1.000 & 15.030 & 286.104 \\
\hline \multirow[t]{4}{*}{ SPEED* CODE } & Sphericity Assumed & 1.052 & 2 & .526 & 20.019 \\
\hline & Greenhouse-Geisser & 1.052 & 1.693 & .621 & 20.019 \\
\hline & Huynh-Feldt & 1.052 & 1.868 & .563 & 20.019 \\
\hline & Lower-bound & 1.052 & 1.000 & 1.052 & 20.019 \\
\hline \multirow[t]{4}{*}{ Error(SPEED) } & Sphericity Assumed & 1.366 & 52 & .026 & \\
\hline & Greenhouse-Geisser & 1.366 & 44.021 & .031 & \\
\hline & Huynh-Feldt & 1.366 & 48.570 & .028 & \\
\hline & Lower-bound & 1.366 & 26.000 & .053 & \\
\hline \multirow[t]{4}{*}{ talk } & Sphericity Assumed & .875 & 1 & .875 & 49.349 \\
\hline & Greenhouse-Geisser & .875 & 1.000 & .875 & 49.349 \\
\hline & Huynh-Feldt & .875 & 1.000 & .875 & 49.349 \\
\hline & Lower-bound & .875 & 1.000 & .875 & 49.349 \\
\hline \multirow[t]{4}{*}{ talk* CODE } & Sphericity Assumed & .057 & 1 & .057 & 3.236 \\
\hline & Greenhouse-Geisser & .057 & 1.000 & .057 & 3.236 \\
\hline & Huynh-Feldt & .057 & 1.000 & .057 & 3.236 \\
\hline & Lower-bound & .057 & 1.000 & .057 & 3.236 \\
\hline \multirow[t]{4}{*}{ Error(talk) } & Sphericity Assumed & .461 & 26 & .018 & \\
\hline & Greenhouse-Geisser & .461 & 26.000 & .018 & \\
\hline & Huynh-Feldt & .461 & 26.000 & .018 & \\
\hline & Lower-bound & .461 & 26.000 & .018 & \\
\hline \multirow[t]{4}{*}{ SPEED * talk } & Sphericity Assumed & .114 & 2 & .057 & 7.876 \\
\hline & Greenhouse-Geisser & .114 & 1.879 & .061 & 7.876 \\
\hline & Huynh-Feldt & .114 & 2.000 & .057 & 7.876 \\
\hline & Lower-bound & .114 & 1.000 & .114 & 7.876 \\
\hline \multirow[t]{4}{*}{ SPEED ${ }^{*}$ talk ${ }^{*}$ CODE } & Sphericity Assumed & .003 & 2 & .001 & .181 \\
\hline & Greenhouse-Geisser & .003 & 1.879 & .001 & .181 \\
\hline & Huynh-Feldt & .003 & 2.000 & .001 & .181 \\
\hline & Lower-bound & .003 & 1.000 & .003 & .181 \\
\hline \multirow[t]{4}{*}{ Error(SPEED*talk) } & Sphericity Assumed & .376 & 52 & .007 & \\
\hline & Greenhouse-Geisser & .376 & 48.854 & .008 & \\
\hline & Huynh-Feldt & .376 & 52.000 & .007 & \\
\hline & Lower-bound & .376 & 26.000 & .014 & \\
\hline
\end{tabular}

Tests of Between-Subjects Effects

Measure: MEASURE_1

Transformed Variable: Average

\begin{tabular}{|l|r|r|r|r|r|r|}
\hline Source & $\begin{array}{c}\text { Type III Sum } \\
\text { of Squares }\end{array}$ & \multicolumn{1}{c|}{ df } & Mean Square & \multicolumn{1}{c|}{ F } & \multicolumn{1}{c|}{ Sig. } & \multicolumn{1}{c|}{$\begin{array}{c}\text { Partial Eta } \\
\text { Squared }\end{array}$} \\
\hline Intercept & 238.376 & 1 & 238.376 & 1410.790 & .000 & .982 \\
CODE & 2.565 & 1 & 2.565 & 15.179 & .001 & .369 \\
Error & 4.393 & 26 & .169 & & & \\
\hline
\end{tabular}




\section{Appendix J. 3 Way ANOVA: Walking Speed and Interlocutor Distance}

\section{General Linear Model}

Index for 3 Way ANOVA: Walking Speed and Interlocutor Distance

*code $1=$ control participants, code $2=$ PD participants, interloc $1=$ one meter interlocutor distance, interloc $2=$ six meters interlocutor distance, slow $1=$ walking slow at one meter interlocutor distance, slow $6=$ walking slow at six meters interlocutor distance, norm $1=$ walking normal at one meter interlocutor distance, norm $6=$ walking normal at six meters interlocutor distance, fast $1=$ walking fast at one meter interlocutor distance, fast $6=$ walking fast at six meters interlocutor distance

Within-Subjects Factors

Measure: MEASURE_1

\begin{tabular}{|ll|l|}
\hline & & $\begin{array}{c}\text { Dependent } \\
\text { Variable }\end{array}$ \\
\hline 1 & 1 & SLOW1 \\
& 2 & SLOW6 \\
\hline 2 & 1 & NORM1 \\
& 2 & NORM6 \\
\hline 3 & 1 & FAST1 \\
& 2 & FAST6 \\
\hline
\end{tabular}

Between-Subjects Factors

\begin{tabular}{|rr|r|}
\hline & & \multicolumn{1}{|c|}{$\mathrm{N}$} \\
\hline CODE & 1.000 & 13 \\
& 2.000 & 12 \\
\hline
\end{tabular}


Descriptive Statistics

\begin{tabular}{|ll|r|r|r|}
\hline & CODE & \multicolumn{1}{c|}{ Mean } & Std. Deviation & \multicolumn{1}{c|}{$\mathrm{N}$} \\
\hline SLOW1 & 1.000 & .83999 & .150242 & 13 \\
& 2.000 & .74940 & .208903 & 12 \\
& Total & .79650 & .182817 & 25 \\
\hline SLOW6 & 1.000 & .84806 & .187075 & 13 \\
& 2.000 & .72251 & .238879 & 12 \\
& Total & .78780 & .218520 & 25 \\
\hline NORM1 & 1.000 & 1.27007 & .191836 & 13 \\
& 2.000 & .95568 & .233712 & 12 \\
& Total & 1.11917 & .262934 & 25 \\
\hline NORM6 & 1.000 & 1.24515 & .151296 & 13 \\
& 2.000 & .93745 & .279762 & 12 \\
& Total & 1.09746 & .268205 & 25 \\
\hline FAST1 & 1.000 & 1.70737 & .140456 & 13 \\
& 2.000 & 1.23724 & .351540 & 12 \\
& Total & 1.48171 & .352096 & 25 \\
\hline FAST6 & 1.000 & 1.65334 & .135149 & 13 \\
& 2.000 & 1.26173 & .367636 & 12 \\
& Total & 1.46537 & .333094 & 25 \\
\hline
\end{tabular}


Tests of Within-Subjects Effects

Measure: MEASURE_1

\begin{tabular}{|c|c|c|c|c|c|}
\hline Source & & $\begin{array}{c}\text { Type III Sum } \\
\text { of Squares }\end{array}$ & df & Mean Square & $\mathrm{F}$ \\
\hline \multirow[t]{4}{*}{ SPEED } & Sphericity Assumed & 11.392 & 2 & 5.696 & 186.844 \\
\hline & Greenhouse-Geisser & 11.392 & 1.463 & 7.788 & 186.844 \\
\hline & Huynh-Feldt & 11.392 & 1.605 & 7.098 & 186.844 \\
\hline & Lower-bound & 11.392 & 1.000 & 11.392 & 186.844 \\
\hline \multirow[t]{4}{*}{ SPEED * CODE } & Sphericity Assumed & .665 & 2 & .332 & 10.900 \\
\hline & Greenhouse-Geisser & .665 & 1.463 & .454 & 10.900 \\
\hline & Huynh-Feldt & .665 & 1.605 & .414 & 10.900 \\
\hline & Lower-bound & .665 & 1.000 & .665 & 10.900 \\
\hline \multirow[t]{4}{*}{ Error(SPEED) } & Sphericity Assumed & 1.402 & 46 & .030 & \\
\hline & Greenhouse-Geisser & 1.402 & 33.641 & .042 & \\
\hline & Huynh-Feldt & 1.402 & 36.914 & .038 & \\
\hline & Lower-bound & 1.402 & 23.000 & .061 & \\
\hline \multirow[t]{4}{*}{ INTERLOC } & Sphericity Assumed & .009 & 1 & .009 & .926 \\
\hline & Greenhouse-Geisser & .009 & 1.000 & .009 & .926 \\
\hline & Huynh-Feldt & .009 & 1.000 & .009 & .926 \\
\hline & Lower-bound & .009 & 1.000 & .009 & .926 \\
\hline \multirow[t]{4}{*}{ INTERLOC $\cdot$ CODE } & Sphericity Assumed & .003 & 1 & .003 & .279 \\
\hline & Greenhouse-Geisser & .003 & 1.000 & .003 & .279 \\
\hline & Huynh-Feldt & .003 & 1.000 & .003 & .279 \\
\hline & Lower-bound & .003 & 1.000 & .003 & .279 \\
\hline \multirow[t]{4}{*}{ Error(INTERLOC) } & Sphericity Assumed & .216 & 23 & .009 & \\
\hline & Greenhouse-Geisser & .216 & 23.000 & .009 & \\
\hline & Huynh-Feldt & .216 & 23.000 & .009 & \\
\hline & Lower-bound & .216 & 23.000 & .009 & \\
\hline \multirow[t]{4}{*}{ SPEED * INTERLOC } & Sphericity Assumed & .001 & 2 & .000 & .028 \\
\hline & Greenhouse-Geisser & .001 & 1.846 & .001 & .028 \\
\hline & Huynh-Feldt & .001 & 2.000 & .000 & .028 \\
\hline & Lower-bound & .001 & 1.000 & .001 & .028 \\
\hline \multirow{4}{*}{$\begin{array}{l}\text { SPEED * INTERLOC * } \\
\text { CODE }\end{array}$} & Sphericity Assumed & .021 & 2 & .010 & .628 \\
\hline & Greenhouse-Geisser & .021 & 1.846 & .011 & .628 \\
\hline & Huynh-Feldt & .021 & 2.000 & .010 & .628 \\
\hline & Lower-bound & .021 & 1.000 & .021 & .628 \\
\hline \multirow[t]{4}{*}{ Error(SPEED*INTERLOC) } & Sphericity Assumed & .753 & 46 & .016 & \\
\hline & Greenhouse-Geisser & .753 & 42.448 & .018 & \\
\hline & Huynh-Feldt & .753 & 46.000 & .016 & \\
\hline & Lower-bound & .753 & 23.000 & .033 & \\
\hline
\end{tabular}

Tests of Between-Subjects Effects

Measure: MEASURE_1

Transformed Variable: Average

\begin{tabular}{|l|r|r|r|r|r|r|}
\hline Source & $\begin{array}{c}\text { Type III Sum } \\
\text { of Squares }\end{array}$ & \multicolumn{1}{c|}{ df } & Mean Square & \multicolumn{1}{c|}{ F } & \multicolumn{1}{c|}{ Sig. } & \multicolumn{1}{c|}{$\begin{array}{c}\text { Partial Eta } \\
\text { Squared }\end{array}$} \\
\hline Intercept & 187.524 & 1 & 187.524 & 878.920 & .000 & .974 \\
CODE & 3.005 & 1 & 3.005 & 14.087 & .001 & .380 \\
Error & 4.907 & 23 & .213 & & & \\
\hline
\end{tabular}




\section{Appendix K. 3 Way ANOVA: Stride Length and Concurrent Talking}

\section{General Linear Model}

Index for 3 Way ANOVA: Stride Length and Concurrent Talking

$*$ code $1=$ control participants, code $2=$ PD participants, speed $1=$ slow, speed $2=$ normal, speed $3=$ fast, talk $1=$ talking and walking, talk $2=$ walking only, slow $1=$ talking while walking at a slow speed, slowNT $=$ walking at slow speed without talking, norm $1=$ talking while walking at normal speed, normNT $=$ walking at normal speed without talking, fast $1=$ talking and walking at fast speed, fastNT $=$ walking at fast speed without talking

Within-Subjects Factors

Measure: MEASURE_1

\begin{tabular}{|ll|l|}
\hline SPEED & talk & $\begin{array}{c}\text { Dependent } \\
\text { Variable }\end{array}$ \\
\hline 1 & 1 & SLOW1 \\
& 2 & SLOWNT \\
\hline 2 & 1 & NORM1 \\
& 2 & NORMNT \\
\hline 3 & 1 & FAST1 \\
& 2 & FASTNT \\
\hline
\end{tabular}

Between-Subjects Factors

\begin{tabular}{|ll|r|}
\hline & & \multicolumn{1}{|c|}{$\mathrm{N}$} \\
\hline CODE & 1.000 & 14 \\
& 2.000 & 14 \\
\hline
\end{tabular}


Descriptive Statistics

\begin{tabular}{|ll|r|r|r|}
\hline & CODE & \multicolumn{1}{c|}{ Mean } & Std. Deviation & \multicolumn{1}{l|}{$\mathrm{N}$} \\
\hline SLOW1 & 1.000 & .83130 & .147963 & 14 \\
& 2.000 & .74857 & .192217 & 14 \\
& Total & .78994 & .173507 & 28 \\
\hline SLOWNT & 1.000 & .85528 & .166066 & 14 \\
& 2.000 & .86606 & .215004 & 14 \\
& Total & .86067 & .188589 & 28 \\
\hline NORM1 & 1.000 & 1.25887 & .189022 & 14 \\
& 2.000 & .94297 & .227029 & 14 \\
& Total & 1.10092 & .260560 & 28 \\
\hline NORMNT & 1.000 & 1.41014 & .122036 & 14 \\
& 2.000 & 1.14908 & .255108 & 14 \\
& Total & 1.27961 & .237013 & 28 \\
\hline FAST1 & 1.000 & 1.69295 & .145332 & 14 \\
& 2.000 & 1.23931 & .323536 & 14 \\
& Total & 1.46613 & .337523 & 28 \\
\hline FASTNT & 1.000 & 1.83987 & .151398 & 14 \\
& 2.000 & 1.45971 & .242523 & 14 \\
& Total & 1.64979 & .277172 & 28 \\
\hline
\end{tabular}


Tests of Within-Subjects Effects

Measure: MEASURE_1

\begin{tabular}{|c|c|c|c|c|c|}
\hline Source & & $\begin{array}{c}\text { Type III Sum } \\
\text { of Squares }\end{array}$ & $d f$ & Mean Square & $\mathrm{F}$ \\
\hline \multirow[t]{4}{*}{ SPEED } & Sphericity Assumed & 15.030 & 2 & 7.515 & 286.104 \\
\hline & Greenhouse-Geisser & 15.030 & 1.693 & 8.877 & 286.104 \\
\hline & Huynh-Feldt & 15.030 & 1.868 & 8.046 & 286.104 \\
\hline & Lower-bound & 15.030 & 1.000 & 15.030 & 286.104 \\
\hline \multirow[t]{4}{*}{ SPEED * CODE } & Sphericity Assumed & 1.052 & 2 & .526 & 20.019 \\
\hline & Greenhouse-Geisser & 1.052 & 1.693 & .621 & 20.019 \\
\hline & Huynh-Feidt & 1.052 & 1.868 & .563 & 20.019 \\
\hline & Lower-bound & 1.052 & 1.000 & 1.052 & 20.019 \\
\hline \multirow[t]{4}{*}{ Error(SPEED) } & Sphericity Assumed & 1.366 & 52 & .026 & \\
\hline & Greenhouse-Geisser & 1.366 & 44.021 & .031 & \\
\hline & Huynh-Feldt & 1.366 & 48.570 & .028 & \\
\hline & Lower-bound & 1.366 & 26.000 & .053 & \\
\hline \multirow[t]{4}{*}{ talk } & Sphericity Assumed & .875 & 1 & .875 & 49.349 \\
\hline & Greenhouse-Geisser & .875 & 1.000 & .875 & 49.349 \\
\hline & Huynh-Feidt & .875 & 1.000 & .875 & 49.349 \\
\hline & Lower-bound & .875 & 1.000 & .875 & 49.349 \\
\hline \multirow[t]{4}{*}{ talk * CODE } & Sphericity Assumed & .057 & 1 & .057 & 3.236 \\
\hline & Greenhouse-Geisser & .057 & 1.000 & .057 & 3.236 \\
\hline & Huynh-Feldt & .057 & 1.000 & .057 & 3.236 \\
\hline & Lower-bound & .057 & 1.000 & .057 & 3.236 \\
\hline \multirow[t]{4}{*}{ Error(talk) } & Sphericity Assumed & .461 & 26 & .018 & \\
\hline & Greenhouse-Geisser & .461 & 26.000 & .018 & \\
\hline & Huynh-Feldt & .461 & 26.000 & .018 & \\
\hline & Lower-bound & .461 & 26.000 & .018 & \\
\hline \multirow[t]{4}{*}{ SPEED* talk } & Sphericity Assumed & .114 & 2 & .057 & 7.876 \\
\hline & Greenhouse-Geisser & .114 & 1.879 & .061 & 7.876 \\
\hline & Huynh-Feldt & .114 & 2.000 & .057 & 7.876 \\
\hline & Lower-bound & .114 & 1.000 & .114 & 7.876 \\
\hline \multirow[t]{4}{*}{ SPEED * talk * CODE } & Sphericity Assumed & .003 & 2 & .001 & .181 \\
\hline & Greenhouse-Geisser & .003 & 1.879 & .001 & .181 \\
\hline & Huynh-Feldt & .003 & 2.000 & .001 & .181 \\
\hline & Lower-bound & .003 & 1.000 & .003 & .181 \\
\hline \multirow[t]{4}{*}{ Error(SPEED*talk) } & Sphericity Assumed & .376 & 52 & .007 & \\
\hline & Greenhouse-Geisser & .376 & 48.854 & .008 & \\
\hline & Huynh-Feldt & .376 & 52.000 & .007 & \\
\hline & Lower-bound & .376 & 26.000 & .014 & \\
\hline
\end{tabular}

Tests of Between-Subjects Effects

Measure: MEASURE_1

Transformed Variable: Average

\begin{tabular}{|l|r|r|r|r|r|r|}
\hline Source & $\begin{array}{c}\text { Type III Sum } \\
\text { of Squares }\end{array}$ & \multicolumn{1}{c|}{ df } & Mean Square & \multicolumn{1}{c|}{ F } & \multicolumn{1}{c|}{ Sig. } & \multicolumn{1}{c|}{$\begin{array}{c}\text { Partial Eta } \\
\text { Squared }\end{array}$} \\
\hline Intercept & 238.376 & 1 & 238.376 & 1410.790 & .000 & .982 \\
CODE & 2.565 & 1 & 2.565 & 15.179 & .001 & .369 \\
Error & 4.393 & 26 & .169 & & & \\
\hline
\end{tabular}




\section{Appendix L. 3 Way ANOVA: Stride Length and Interlocutor Distance}

\section{General Linear Model}

Index for 3 Way ANOVA: Stride Length and Interlocutor Distance

*code $1=$ control participants, code $2=$ PD participants, interloc $1=$ one meter interlocutor distance, interloc $2=$ six meters interlocutor distance, slow $1=$ walking slow at one meter interlocutor distance, slow $6=$ walking slow at six meters interlocutor distance, norm $1=$ walking normal at one meter interlocutor distance, norm $6=$ walking normal at six meters interlocutor distance, fast $1=$ walking fast at one meter interlocutor distance, fast $6=$ walking fast at six meters interlocutor distance

Within-Subjects Factors

Measure: MEASURE_1

\begin{tabular}{|ll|l|}
\hline SPEED & INTERLOC & $\begin{array}{c}\text { Dependent } \\
\text { Variable }\end{array}$ \\
\hline 1 & 1 & SLOW1 \\
& 2 & SLOW6 \\
\hline 2 & 1 & NORM1 \\
& 2 & NORM6 \\
\hline 3 & 1 & FAST1 \\
& 2 & FAST6 \\
\hline
\end{tabular}

Between-Subjects Factors

\begin{tabular}{|ll|r|}
\hline & & \multicolumn{1}{|c|}{$\mathrm{N}$} \\
\hline CODE & 1.000 & 13 \\
& 2.000 & 12 \\
\hline
\end{tabular}




\section{Descriptive Statistics}

\begin{tabular}{|ll|r|r|r|}
\hline & CODE & Mean & Std. Deviation & \multicolumn{1}{c|}{$\mathrm{N}$} \\
\hline SLOW1 & 1.000 & .83999 & .150242 & 13 \\
& 2.000 & .74940 & .208903 & 12 \\
& Total & .79650 & .182817 & 25 \\
\hline SLOW6 & 1.000 & .84806 & .187075 & 13 \\
& 2.000 & .72251 & .238879 & 12 \\
& Total & .78780 & .218520 & 25 \\
\hline NORM1 & 1.000 & 1.27007 & .191836 & 13 \\
& 2.000 & .95568 & .233712 & 12 \\
& Total & 1.11917 & .262934 & 25 \\
\hline NORM6 & 1.000 & 1.24515 & .151296 & 13 \\
& 2.000 & .93745 & .279762 & 12 \\
& Total & 1.09746 & .268205 & 25 \\
\hline FAST1 & 1.000 & 1.70737 & .140456 & 13 \\
& 2.000 & 1.23724 & .351540 & 12 \\
& Total & 1.48171 & .352096 & 25 \\
\hline FAST6 & 1.000 & 1.65334 & .135149 & 13 \\
& 2.000 & 1.26173 & .367636 & 12 \\
& Total & 1.46537 & .333094 & 25 \\
\hline
\end{tabular}


Tests of Within-Subjects Effects

Measure: MEASURE_1

\begin{tabular}{|c|c|c|c|c|c|}
\hline Source & & $\begin{array}{c}\text { Type III Sum } \\
\text { of Squares }\end{array}$ & df & Mean Square & $\mathrm{F}$ \\
\hline \multirow[t]{4}{*}{ SPEED } & Sphericity Assumed & 11.392 & 2 & 5.696 & 186.844 \\
\hline & Greenhouse-Geisser & 11.392 & 1.463 & 7.788 & 186.844 \\
\hline & Huynh-Feldt & 11.392 & 1.605 & 7.098 & 186.844 \\
\hline & Lower-bound & 11.392 & 1.000 & 11.392 & 186.844 \\
\hline \multirow[t]{4}{*}{ SPEED $\cdot$ CODE } & Sphericity Assumed & .665 & 2 & .332 & 10.900 \\
\hline & Greenhouse-Geisser & .665 & 1.463 & .454 & 10.900 \\
\hline & Huynh-Feldt & .665 & 1.605 & .414 & 10.900 \\
\hline & Lower-bound & .665 & 1.000 & .665 & 10.900 \\
\hline \multirow[t]{4}{*}{ Error(SPEED) } & Sphericity Assumed & 1.402 & 46 & .030 & \\
\hline & Greenhouse-Geisser & 1.402 & 33.641 & .042 & \\
\hline & Huynh-Feldt & 1.402 & 36.914 & .038 & \\
\hline & Lower-bound & 1.402 & 23.000 & .061 & \\
\hline \multirow[t]{4}{*}{ INTERLOC } & Sphericity Assumed & .009 & 1 & .009 & .926 \\
\hline & Greenhouse-Geisser & .009 & 1.000 & .009 & .926 \\
\hline & Huynh-Feldt & .009 & 1.000 & .009 & .926 \\
\hline & Lower-bound & .009 & 1.000 & .009 & .926 \\
\hline \multirow[t]{4}{*}{ INTERLOC * CODE } & Sphericity Assumed & .003 & 1 & .003 & .279 \\
\hline & Greenhouse-Geisser & .003 & 1.000 & .003 & .279 \\
\hline & Huynh-Feldt & .003 & 1.000 & .003 & .279 \\
\hline & Lower-bound & .003 & 1.000 & .003 & .279 \\
\hline \multirow[t]{4}{*}{ Error(INTERLOC) } & Sphericity Assumed & .216 & 23 & .009 & \\
\hline & Greenhouse-Geisser & .216 & 23.000 & .009 & \\
\hline & Huynh-Feldt & .216 & 23.000 & .009 & \\
\hline & Lower-bound & .216 & 23.000 & .009 & \\
\hline \multirow[t]{4}{*}{ SPEED* INTERLOC } & Sphericity Assumed & .001 & 2 & .000 & .028 \\
\hline & Greenhouse-Geisser & .001 & 1.846 & .001 & .028 \\
\hline & Huynh-Feldt & .001 & 2.000 & .000 & .028 \\
\hline & Lower-bound & .001 & 1.000 & .001 & .028 \\
\hline \multirow{4}{*}{$\begin{array}{l}\text { SPEED * INTERLOC* } \\
\text { CODE }\end{array}$} & Sphericity Assumed & .021 & 2 & .010 & .628 \\
\hline & Greenhouse-Geisser & .021 & 1.846 & .011 & .628 \\
\hline & Huynh-Feldt & .021 & 2.000 & .010 & .628 \\
\hline & Lower-bound & .021 & 1.000 & .021 & .628 \\
\hline \multirow[t]{4}{*}{ Error(SPEED*INTERLOC) } & Sphericity Assumed & .753 & 46 & .016 & \\
\hline & Greenhouse-Geisser & .753 & 42.448 & .018 & \\
\hline & Huynh-Feldt & .753 & 46.000 & .016 & \\
\hline & Lower-bound & .753 & 23.000 & .033 & \\
\hline
\end{tabular}

Tests of Between-Subjects Effects

Measure: MEASURE_1

Transformed Variable: Average

\begin{tabular}{|l|r|r|r|r|r|r|}
\hline Source & $\begin{array}{c}\text { Type III Sum } \\
\text { of Squares }\end{array}$ & \multicolumn{1}{c|}{ df } & Mean Square & \multicolumn{1}{c|}{ F } & \multicolumn{1}{c|}{ Sig. } & \multicolumn{1}{c|}{$\begin{array}{c}\text { Partial Eta } \\
\text { Squared }\end{array}$} \\
\hline Intercept & 187.524 & 1 & 187.524 & 878.920 & .000 & .974 \\
CODE & 3.005 & 1 & 3.005 & 14.087 & .001 & .380 \\
Error & 4.907 & 23 & .213 & & & \\
\hline
\end{tabular}




\title{
Curriculum Vitae
}

\author{
CASSANDRA M. MCCAIG, M.Sc. Candidate \\ Faculty of Health and Rehabilitation Sciences \\ Western University Canada
}

School Mailing Address:

School of Communication Sciences \& Disorders, Elborn College, University of Western Ontario,

London, Ontario, N6G 1H1

Home Mailing Address:
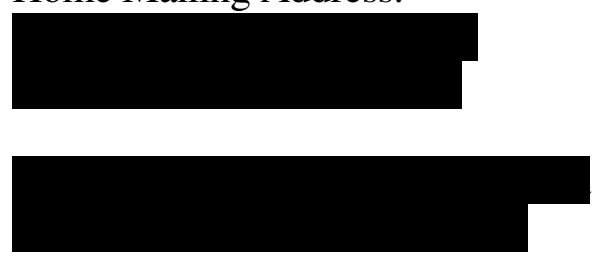

\section{EDUCATION}

Undergraduate Bachelor of Arts (Honours Specialization) in Kinesiology, Western University Canada. 2007-2011

Graduate Master of Science in Health and Rehabilitation Sciences, Western University Canada (Supervisor, Dr. Scott Adams). 2011-Current

\section{CONFERENCE PRESENTATIONS}

Schell, M., McCaig, C., Nivins, M., Jog, M., Adams, S. Vowel Formant Dynamics Across Changes in Speech Intensity in Parkinson's Disease. Aging, Rehabilitation \& Geriatric Care Research/Faculty of Health Science (ARGC/FHS) Symposium, Western University Canada, February 2012

McCaig C., Schell, M., Nivins, M., Jog, M., Adams, S. Vowel Formant Dynamics Across Changes in Speech Intensity in Parkinson's Disease. Health and Rehabilitation Sciences Graduate Research Forum, Western University Canada, February 2012

McCaig C., Schell, M., Nivins, M., Jog, M., Adams, S. Vowel Formant Dynamics Across Changes in Speech Intensity in Parkinson's Disease. Western Research Forum, Western University Canada, March 2012

McCaig C., Schell, M., Nivins, M., Jog, M., Adams, S. Vowel Formant Dynamics Across Changes in Speech Intensity in Parkinson's Disease. The Southern Ontario Interdisciplinary Health \& Aging Symposium, Hamilton Ontario, March 2012 
McCaig C., Schell, M., Nivins, M., Jog, M., Adams, S. Vowel Formant Dynamics Across Changes in Speech Intensity in Parkinson's Disease. Faculty of Health Sciences Research Day, Western University Canada, April 2012

McCaig, C., Adams, S., Dykstra, A., \& Jog, M. Effects of concurrent walking and interlocutor distance on conversational speech intensity and rate in Parkinson's disease. The ARGC/FHS Symposium "Research to action: Technology, innovation \& health.” Western University, London, ON. February 1, 2013.

McCaig, C., Adams, S., Dykstra, A., \& Jog, M. Effects of concurrent walking and interlocutor distance on conversational speech intensity and rate in Parkinson's disease. The HRS Graduate Research Forum "Brewing Research, Steeped in Ideas." Western University, London, ON. February 8, 2012.

McCaig, C., Adams, S., Dykstra, A., \& Jog, M. Effects of concurrent walking and interlocutor distance on conversational speech intensity and rate in Parkinson's disease. The Faculty of Health Sciences Research Day. Western University, London, ON. March 13, 2013

\title{
ACADEMIC EXPERIENCE
}

2011 Teaching Assistant School of Occupational Therapy, Western University Canada. Course: OT9531 Conditions and Occupational Performance. Responsibilities included holding office hours, proctoring exams, and marking exams.

2012 Teaching Assistant School of Communication Sciences and Disorders, Western University Canada. Course: CSD9615 Speech Science. Responsibilities include preparing and running labs, proctoring exams, and marking exams.

2012-2013 Field Mentor Health and Rehabilitation Sciences, Western University Canada. Field: Speech and Language Science. Responsibilities are to provide guidance and mentorship for the incoming Health and Rehabilitation graduate students, and to answer any administrative, program related or community-based questions.

\section{COMMUNITY INVOLVMENT}

\begin{abstract}
2012-2013 Health and Rehabilitation Sciences Research Forum Committee Member Health and Rehabilitation Sciences, Western University Canada. Responsibilities include planning and organizing the 2012 Health and Rehabilitation research forum.
\end{abstract}




\section{SCHOLARSHIPS AND AWARDS}

\section{Academic}

\section{0-2011 Academic Scholar - Dean's Honour List}

2011-2012 Western Graduate Research Scholarship (value: \$10,000) Awarded to graduate students who are enrolled in Doctoral programs and Master's programs that prepare students for entry into Doctoral programs. Funding Period: September 2011 August 2012.

2012-2013 Western Graduate Research Scholarship (value: \$10,000) Awarded to graduate students who are enrolled in Doctoral programs and Master's programs that prepare students for entry into Doctoral programs. Funding Period: September 2012August 2013. 